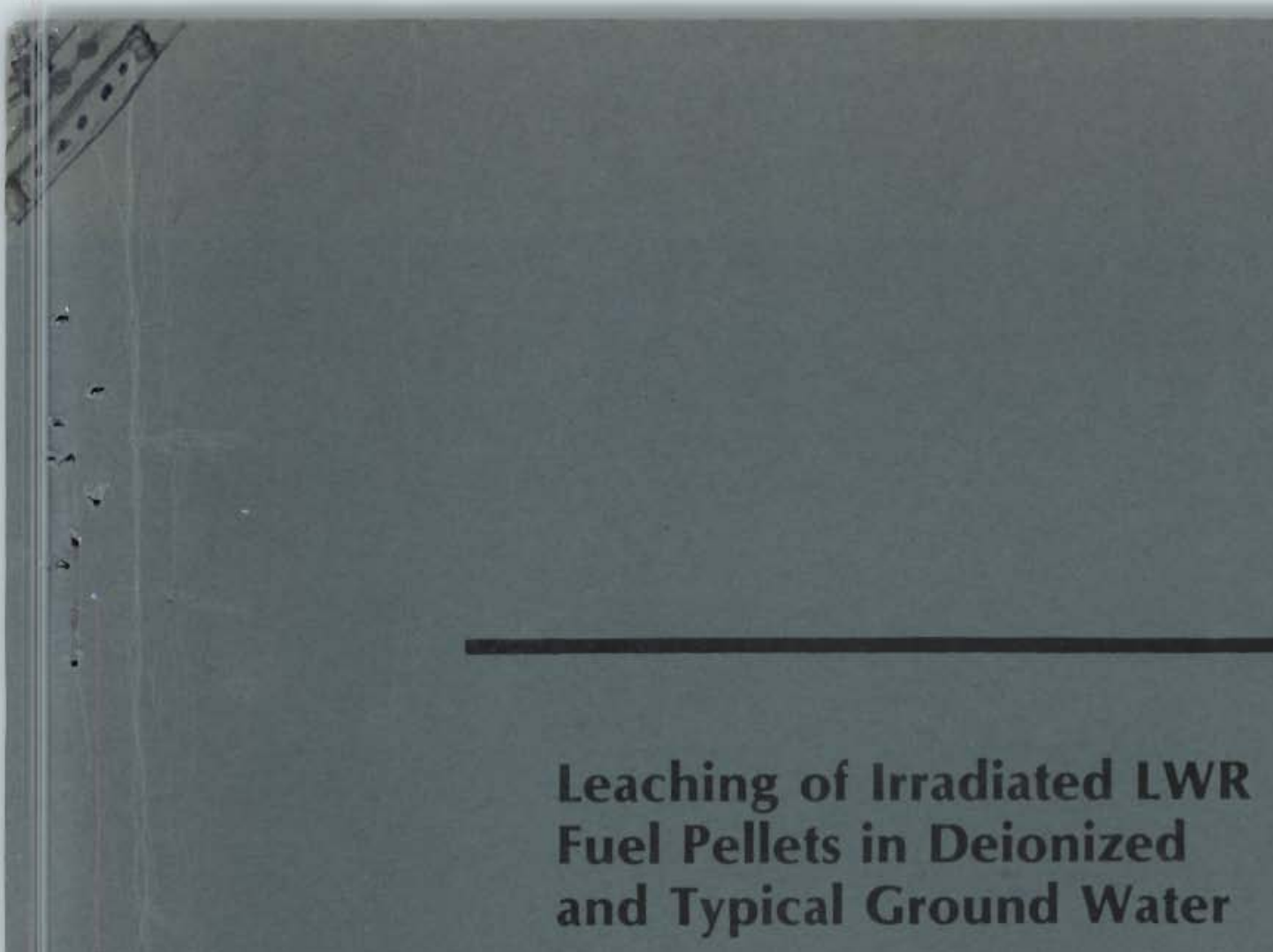

\title{
Leaching of Irradiated LWR Fuel Pellets in Deionized and Typical Ground Water
}

July 1976

Prepared for the Energy Research and Development Administration under Contract E(45-1)-1830

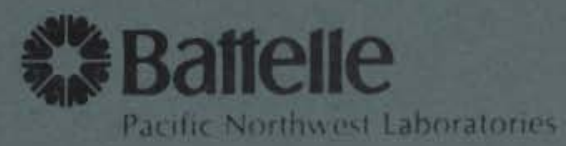


This report was prepared as an account of work sponsored by the United States Government. Neither the United States nor the United States Energy Research and Development Administration, nor any of their employees, nor any of their contractors, subcontractors, or their employees, makes any warranty, express or implied, or assumes any legal liability or responsibility for the accuracy, completeness or usefulness of any information, apparatus, product or process disclosed, or represents that its use would not infringe privately owned rights.

\title{
PACIFIC NORTHWEST LABORATORY \\ operated by \\ BATTELLE \\ for the
}

U.S. ENERGY RESEARCH AND DEVELOPMENT ADMINISTRATION

Under Contract AT(45-1)-1830

\author{
Printed in the United States of America \\ Available from \\ National Technical Information Service \\ U.S. Department of Commerce \\ 5285 Port Royal Road \\ Springtield, Virginia 22151 \\ Price: Printed Copy 57.60 ; Microliche $\mathbf{3 2 . 2 5}$
}


BNWL -2057

\section{3}

LEACHING OF IRRADIATED LWR. FUEL

PELLETS IN DEIONIZED ANN TYPICAL

GROUND WATER

Y. B. Katayama

July 1976

Rattelle-Northwest

Pacific Northwest Laboratories

This paper is based on work performed under 11.S. Enerqy Research and Development Administration Contract $E(45-1): 1837$. 


\section{TABLE OF CONTENTS}

IHTRODUCTION $\cdot$ •

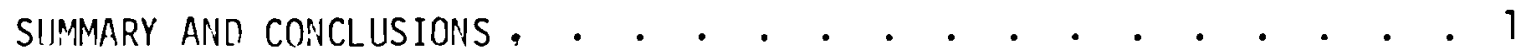

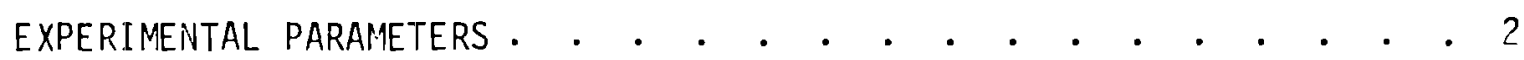
Material • • . . . . . . . . . . . . . . . . . . 2 Equipment . . . . . . . . . . . . . . . . . . . . . 4 Procedure . . . . . . . . . . . . . . . . . . . . 4 EXPERIMENTAL RESULTS AND DISCUSSION • • • • • Calculations Leach Rate • . . . . . . . . . . . . . . . 3 Leach Mechanisms . . . . . . . . . . . . . . . . . 10

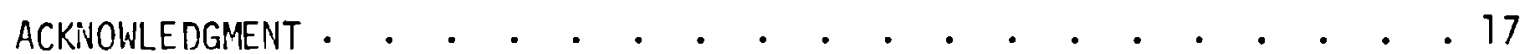

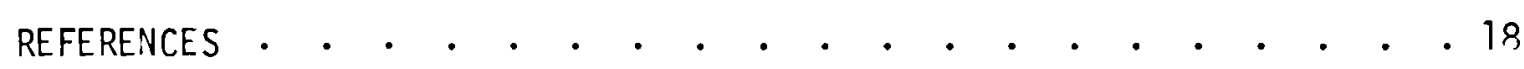

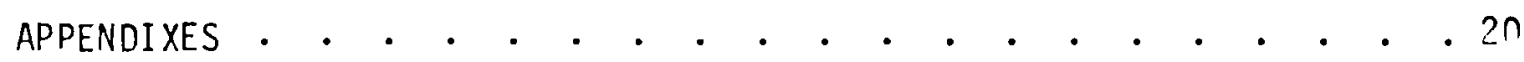




\section{$\underline{\text { INTRODUCTION }}$}

This report presents results of leach test experiments on enriched, $5.81 \mathrm{wt} \%{ }^{235} \mathrm{U}, \mathrm{UO}_{2}$ fuel pellet fragments removed from 1 ight water reactor (LWR.) fuel rods irradiated to an average hurnup of $54,45 n$ MWN/MTII. These results are discussed and correlated with similar information about common solidified high-level waste forms. Leach test data were needer to evaluate the relative radiological hazards of storina irradiated fuel rods.

\section{SUMMARY AND CONCLUSIONS}

Leach tests were performed on irradiated LWR fuel pellet fragments at $25^{\circ} \mathrm{C}$ in static Hanford ground water, deionized water and buildina distilled water. The following conclusions were made:

- The relative leachability of the elements decrease in the order of

$$
\mathrm{Cs}>\mathrm{Sb}>\mathrm{Sr}+\mathrm{Y}>\mathrm{Pu}>\mathrm{Cm} \text {. }
$$

- The leach rates, $g$ of solid $/ \mathrm{cm}^{2}$-day, normalized to behavior of specific radioisotopes have the following value after the indicated weeks of leaching:

\section{Hanford Ground . Water (21 weeks)}

$$
\text { Cs }-137
$$

$\mathrm{Sb}-125$

$\mathrm{Sr}-90+\mathrm{Y}-90$

$\mathrm{Pu}-239+240$

$\mathrm{Cm}-244$

$$
5 \times 10^{-6}
$$

$1 \times 10^{-6}$

$4 \times 10^{-7}$

$1 \times 10^{-8}$

$4 \times 10^{-9}$
Buil.ding Distilled Water (21 weeks)

$5 \times 10^{-5}$

$2 \times 10^{-6}$

$1 \times 10^{-6}$

$4 \times 10^{-7}$

$5 \times 1 n^{-8}$
Deionized Water (20 weeks)

$7 \times 10^{-6}$

$2 \times 10^{-6}$

$1 \times 10^{-6}$

$3 \times 10^{-7}$

$4 \times 10^{-9}$

The leach tests are continuing and the leach rates are expected to level out at a value lower than the ones reported. 
- Two release mechanisms were observed for each radioisotope over the time interval studied. The short term mechanism was linear for ${ }^{244} \mathrm{Cm}$ and logarithmic for the other radioisotopes. This short term mechanism ranged in duration from 5 to 31 days. In every case the long term mechanism was logarithmic and could be fitted to the following expression:

$$
\text { Fraction released }=B t^{m} \text {. }
$$

The value for $m$ was found to be 0.07 for Hanford around water, 0.31 for deionized water, and 0.06 to 0.35 for building distilled water.

- The deionized water was a reference leachant. The Hanford ground water represented typical arid ground water where ionic species retarded the effective diffusional release of the radioisotope to the leachant. The building distilled water was a comparison leachant to the WSEP leach tests and resulted in intermediate leach rates.

- The cesium based periodic leach rate for irradiated fuel fraqments are similar to the cesium based leach rate for borosilicate alass containina radioactive waste, WSEP me1t SS-13.

\section{EXPERIMENTAL PARAMETERS}

\section{Material}

The material used in this investigation was fuel fragments removed from LWR fuel rods irradiated to an average burnup of 54,450 MWD/MTII. This 5.81 wt\% 235 "l enriched fuel had a pre-irradiation composition as given in Table 1, and the geometric density was $93.6 \%$ of theoretical density. The initial irradiation date was necember, 1968, and the discharge date was 0ctober, 1973, with a total of $32,80 n$ hours irradiation which includes 27,800 hours at effective full power. 


\section{TABLE 1. CHEMICAL ANALYSIS OF FUEL PELLETS (5.81 w/O !-235 Enrichment)}

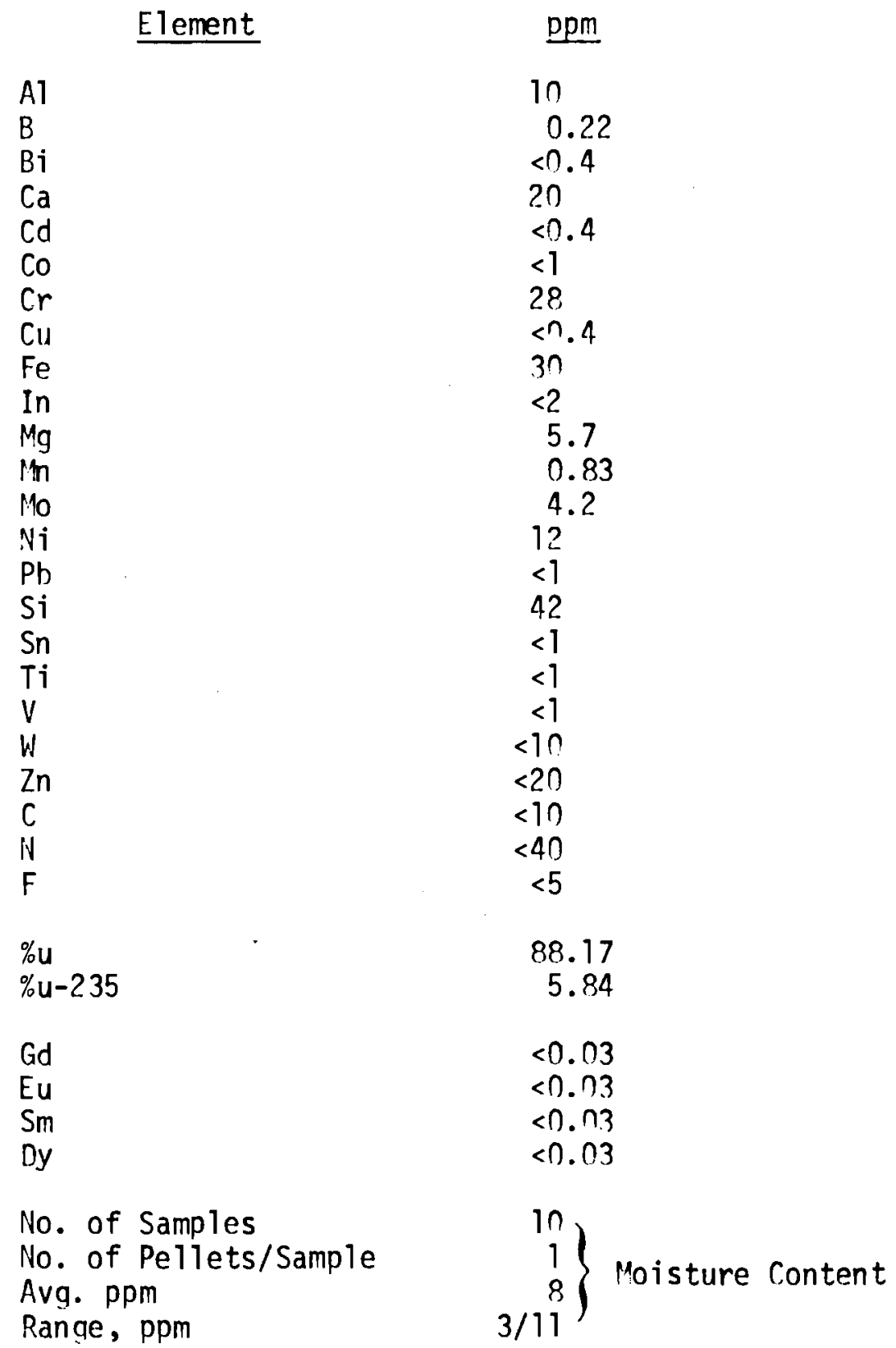




\section{Equipment}

A leach apparatus of the type devised by Paige ${ }^{(1)}$, shown in Fiqure 1 , was used for leaching the fuel fragments. The same apparatus had heen used previously for leaching radioactive solidified wastes from the laste Solidification Enaineering Prototype (WSEP) program at the Pacific llorthwest Laboratories. (2) For comparison purposes, huilding distilled water was one of the leach liquids investiaated. The other leachants vere deionized water and Hanford qround water, representine a tvpical arid region ground water composition, Tahle?.

\section{TABLE 2. GROUND WATER COMPOSITION III PPM IMPIJRITY}

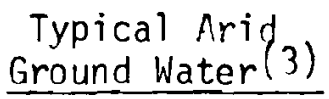

Sulfate

Nitrate

Chloride

Picarbonate

Sodium

Potassium

Calcium

Magnesium

pH
15

1

8

125

20

5

25

5
Hanford Ground Water

20

4.2

3.1

168

25

5.4

31

7.1
$6.8-8.2$
7.9

All the tests were done in ambient temperatures of approximately $25^{\circ} \mathrm{C}$ within $\mathrm{C}-\mathrm{Ce} 11$, a radiochemical enqineerina cell in the $3 ? 4$ Buildina.

Procedure

Large fuel fragment particles were selected for the leach tests in batches of 4 to 5 gram size. These fragment batches were photographed, dimensionally measured and weighed in a tared stainless steel mesh basket. The basket was loaded into a leach apparatus and the leachant added on a londay to accommodate the preplanned sampling schedule. 


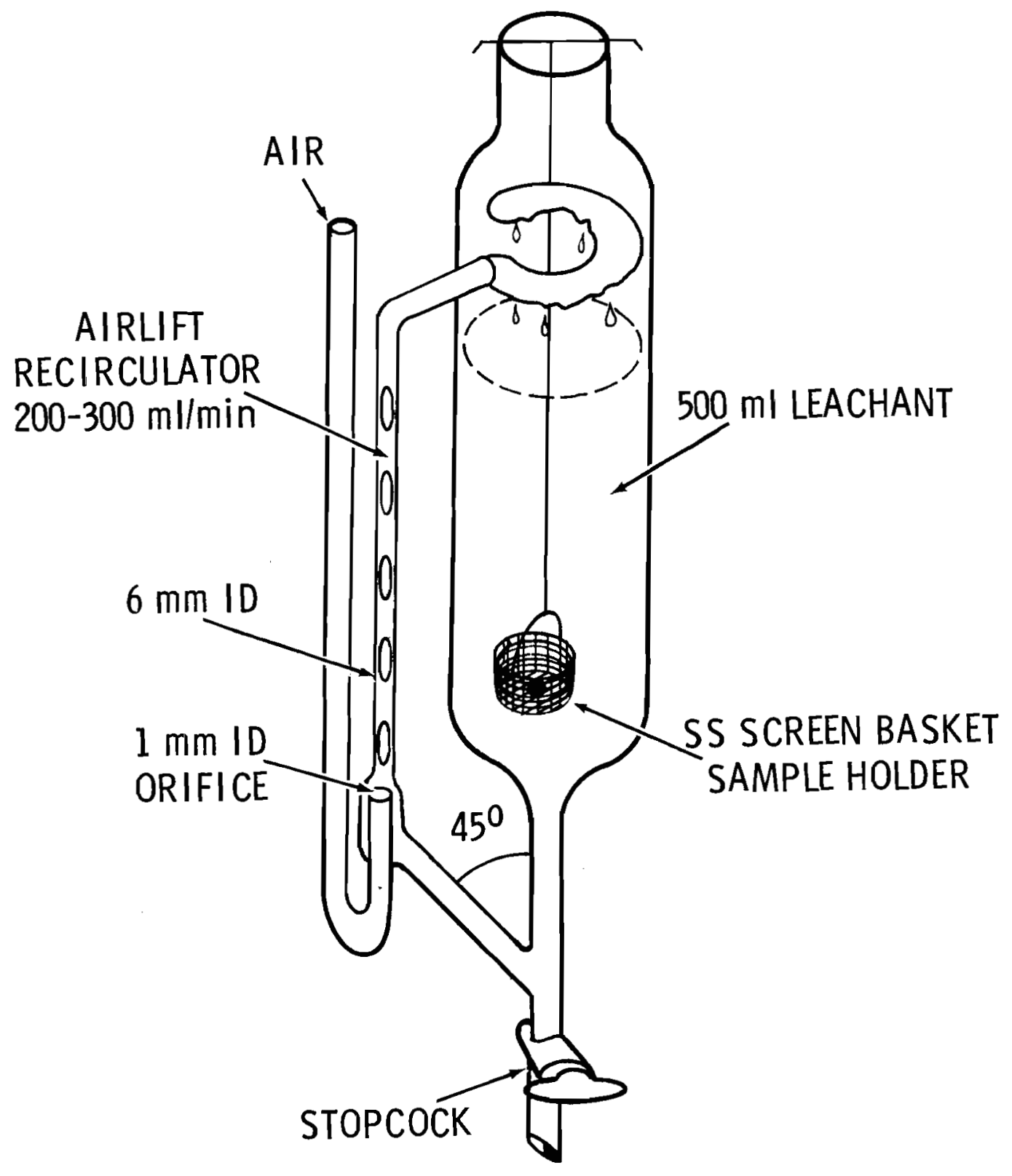

FIGURE 1. Slow-Leach Test Used at the Pacific Northwest Laboratory 
Analytical samples of leachant were taken at the following intervals: 1 day, 1 day, 1 day, 1 day, 3 days, 7 days, 7 days, 7 days, 30 days, 30 days, 30 days. After each sampling, the leach apparatus was drained and fresh leachant added.

\section{EXPERIMENTAL RESIILTS ANI DISCUSSIDN}

\section{Calculations}

The periodic leach rate, $\mathrm{Rp}$, which has the dimensions of arams of solid/ $\mathrm{cm}^{2}$-day was calculated from the following expression:

$$
R_{P}=\frac{\Lambda p}{A 0} \frac{W}{S T p}
$$

where $A p=$ radioactivity of isotope leached during leachant renewal period, $p$

$A o=$ radioactivity of isotope initially present in sample

$S$ = surface area of sample $\left(\mathrm{cm}^{2}\right)$

$W=$ weight of sample (grams)

$\mathrm{Tp}=$ duration (days) of leach period.

The radioactivity, $A p$, was determined by radiochemical analyses. Measurements for ${ }^{137} \mathrm{Cs}$ and ${ }^{134} \mathrm{Cs}$ activities were made by gamma scan analysis of the leach solution. The high concentration of radioactive cesium interferred with the accurate counting of other aamma emitting radioisotopes. Cesium was extracted from the leach solution with tetraphenylboron after complexing the other elements with ethylenediaminetetraacetic acid salts in a basic solution. This step lowered the cesium activity sufficiently for the counting of ${ }^{125} \mathrm{Sb},{ }^{154} \mathrm{Eu},{ }^{144} \mathrm{Ce}$ and ${ }^{106} \mathrm{Ru}$. The actinides, ${ }^{239} \mathrm{Pu}+{ }^{240} \mathrm{Pu},{ }^{242} \mathrm{~cm},{ }^{244} \mathrm{Cm}$ and ${ }^{241} \mathrm{Am}+{ }^{238} \mathrm{Pu}$ were extracted from the leach solution sample and quantified by alpha eneray analysis. Interference of the ${ }^{241} \mathrm{Am}$ determination by ${ }^{238} \mathrm{Pu}$ is normally corrected by determining the ${ }^{238} \mathrm{Pu}$ to ${ }^{239} \mathrm{Pu}+{ }^{240} \mathrm{Pu}$ ratio and applying a correction factor to the ${ }^{241} \mathrm{Am}+{ }^{238} \mathrm{Pu}$ readinas. However this method was not applicable for the fuel frament leach solutions hecause the Ao 
value for ${ }^{238} \mathrm{Pu}$ increases with time. The ${ }^{241} \mathrm{Am}$ was selectively separated from ${ }^{238} \mathrm{Pu}$ and alpha counted in one of the deionized water leach test series, Z-6. ${ }^{90} \mathrm{Sr}+{ }^{90} Y$ concentrations were determined by countina after solution stripping.

The radioactivity of isotopes initially present in the sample, Ao, were taken from the ORIGEN code. (4) This special ORIGEN code printout included the applicable irradiation and post-irradiation history for the fuel pellet fragments used in the leach tests. (5) The use of a constant Ao assumed uniform distribution of activity in the irradiated fuel pellet. Cesium and antimony have been found to be enriched on surfaces of gaps, cracks and pores in irradiated oxide fuel pins and have been occasionally detected in the central void of irradiated oxide fuels. ( $(5)$ Newly broken surfaces free of this enrichment may have been formed during fuel fragment removal.

The surface area of the sample, $S$, was approximated by selectively measuring the dimensions of some of the large particles and applying geometric approximations to photographs of the particles. Examination of the as-received fuel fragments in the hot cell revealed rough dull surfaces typical of high surface solids. Mendel ${ }^{(7)}$ has reported that the leach rate of radioisotopes in high surface solids when expressed in $\mathrm{g} / \mathrm{cm}^{2}$-day usually yield relatively high values, up to $80 n$ n times greater, when the geometric surface area is used in place of the actual surface area. This effect would give maximum leach rate values. Tingey and $\mathrm{Felix}(8,9)$ have reported on the radiolysis of water by the radiation from hiah-level waste. The rough textured fracture surface would be amenable for accumulation of adsorbed gases from the radiolytic decomposition of static leachant at the pellet fragment-water interface. This accumulation would then decrease the effective area for diffusion of ions and would have the effect of giving lower leach rate values. Figure 2 is a photograph of the fuel fragments for run $Z-5$ as viewed through the C-Cell periscope. 


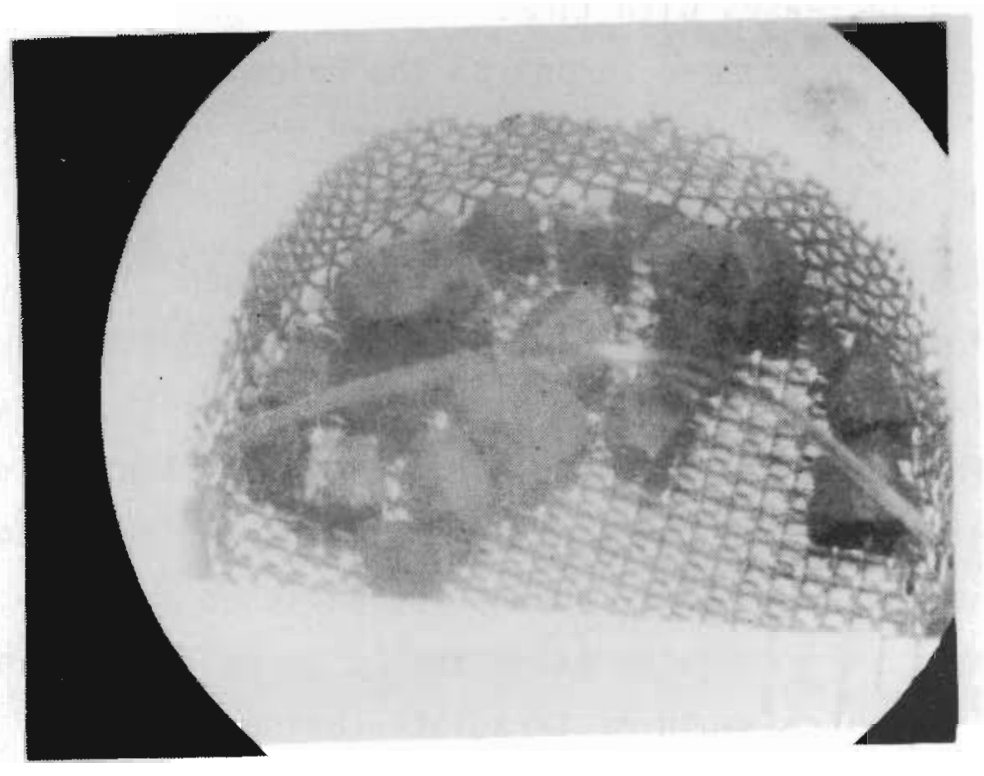

FIGURE 2. Fuel fragments. Leach test run $Z-5$.

The weiaht, $W$, of the sample was measured at the start of the leach testing and will be weighed at the termination of the leach program. Periodic measurements, at various leachant renewal periods, were not made. The complexity of remotely weighing samples and the chances for chemical and physical changes at the leachina surfaces durina dryino were all affimations of this decision.

The duration of leachant renewal period. Tp, was chosen to coincide with the recommended sampling frequency proposed by Hespe. (10) An exception was the shortenting of the weekly samplina period from $?$ to 3 weeks, primarily for economic reasons.

\section{Leach Rates}

Figure 3 is a araph, based on ${ }^{137}$ rs release, where the periodic leach rate is plotted against the days of cumulative leaching. Leach rate. trend lines for the three leachants, Hanford around water, buildina distilled water, and deionized water, are plotted on this araph alono with 


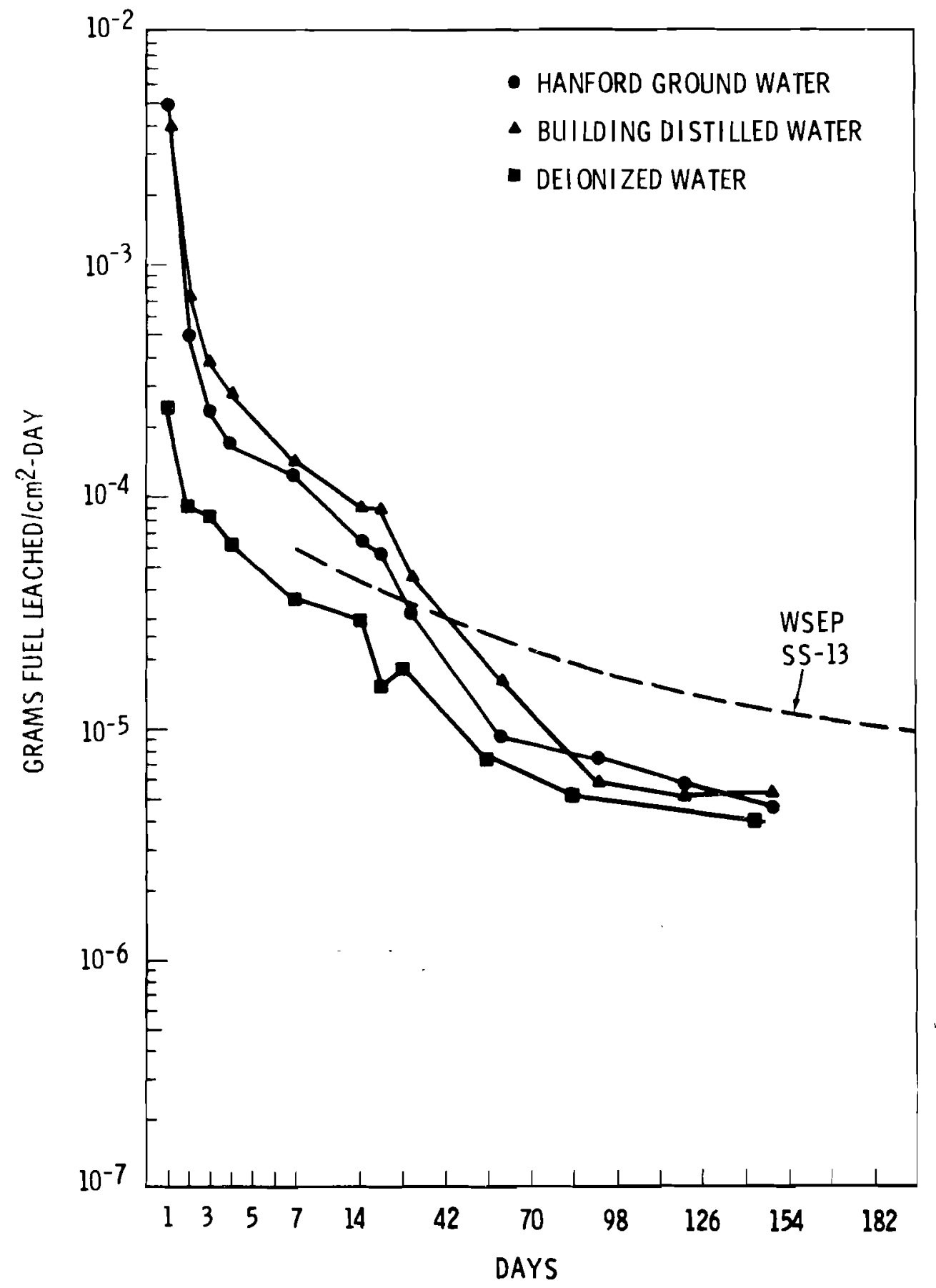

FIGURE 3. Leach Rate of Irradiated Fuel Pellet Based on ${ }^{137} \mathrm{Cs}$ 
the leach rate curve for an in-can-melted borosilicate alass from WSEP run S5-13. (11) The borosilicate glass leach rate curve, based on cesium analysis, approximates the cesium based periodic leach rate for fuel fragments.

The periodic leach rate data for all the radioisotopes analyzed are tabulated in the appendix. Selective leaching of the radioisotopes is evident upon examination of the periodic leach rate data. The leach rate based on cesium is 1000 times greater than the curium based leach rate with the other reported elements spread in between. This unequal leach rate of certain elements has been reported by many investigators and was tabulated for many different forms of solidified waste by Mendel. (7) The relative leachability of elements for the irradiated fuel has been added to this tabulation, Table 3 .

\section{Leach Mechanisms}

Fiqures 4 to 7 are graphs in which the fraction of radioisotopes released are plotted against the days of cumulative leach. These logarithmic graphs show that a combination of different mechanisms have taken place. The obvious curved portion of the logarithmic plot for ${ }^{244} \mathrm{Cm}$ was found to be of linear behavior and can be represented by the general equation:

$$
\mathrm{F}_{\mathrm{R}}=n t+\mathrm{a}
$$

where $F_{R}$ is fraction curium released, $t$ is time (days) and $n$ and a are the slope and intercept of the curve, respectively. The data points below the dashed portion of the curve represent a sample surface conditioning anomaly.

The transition from the initial to the lona term mechanism occurred in 5 to 31 days. In all cases the lona time mechanism is linear on the logarithmic plot and can be represented by the neneral equation:

$$
\log F_{p}=m \log t+b
$$




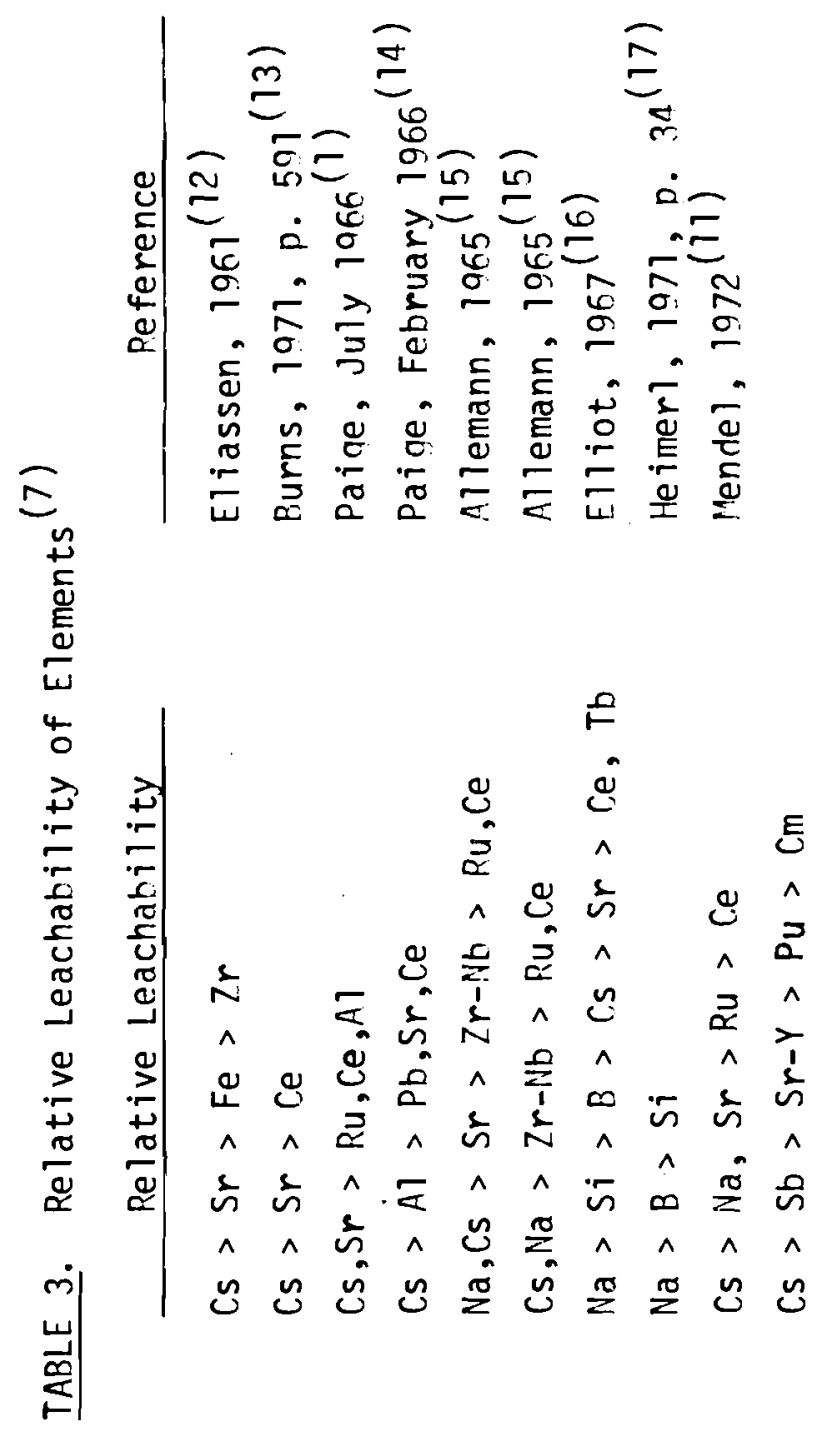

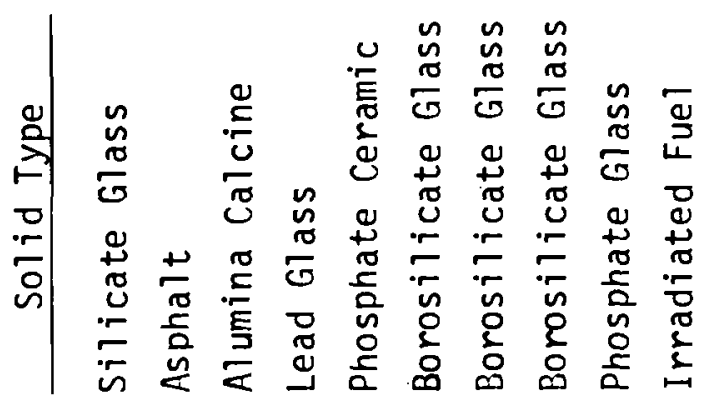




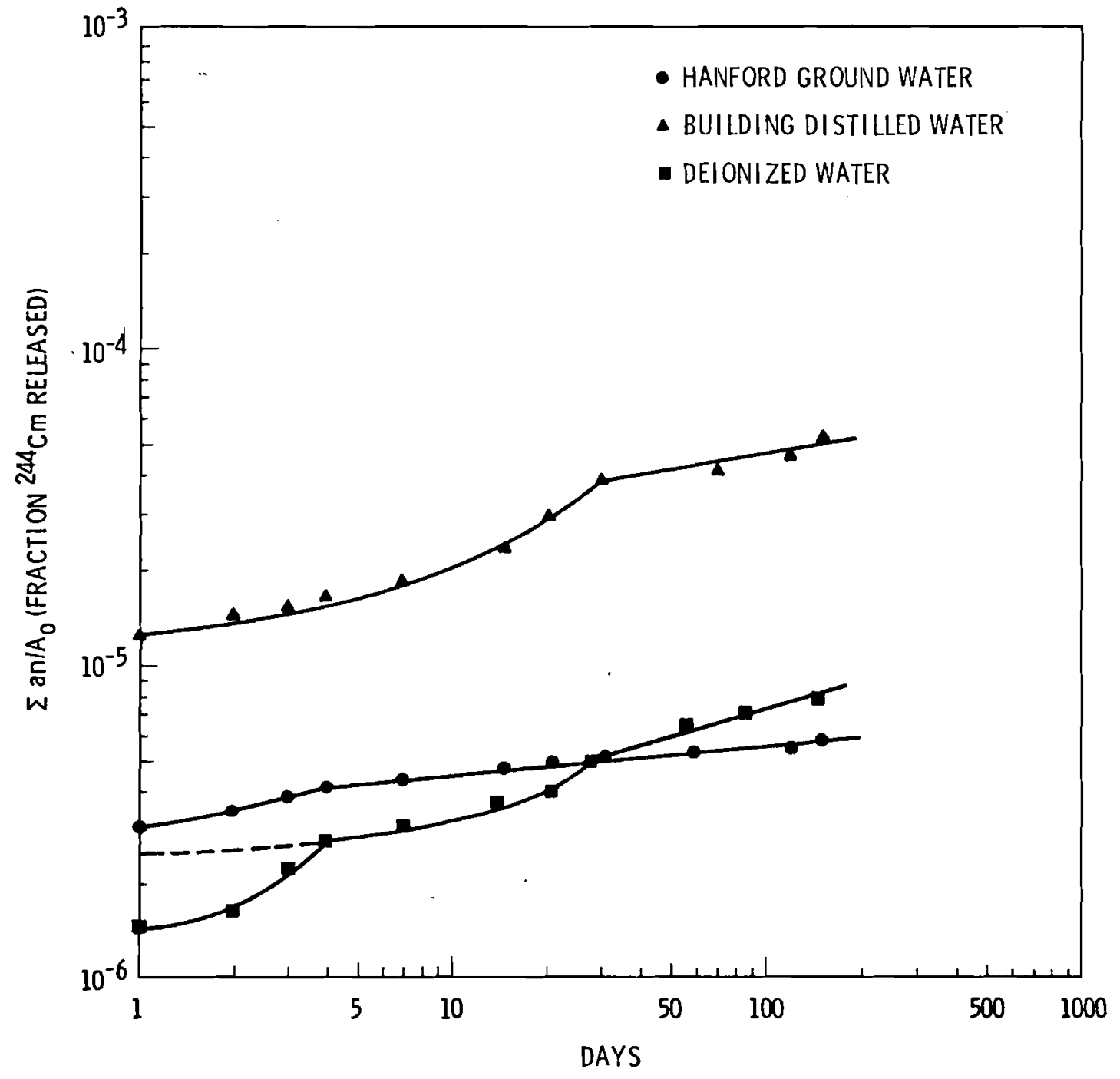

FIGURE 4. Fraction of ${ }^{244} \mathrm{Cm}$ Released to Leachant 


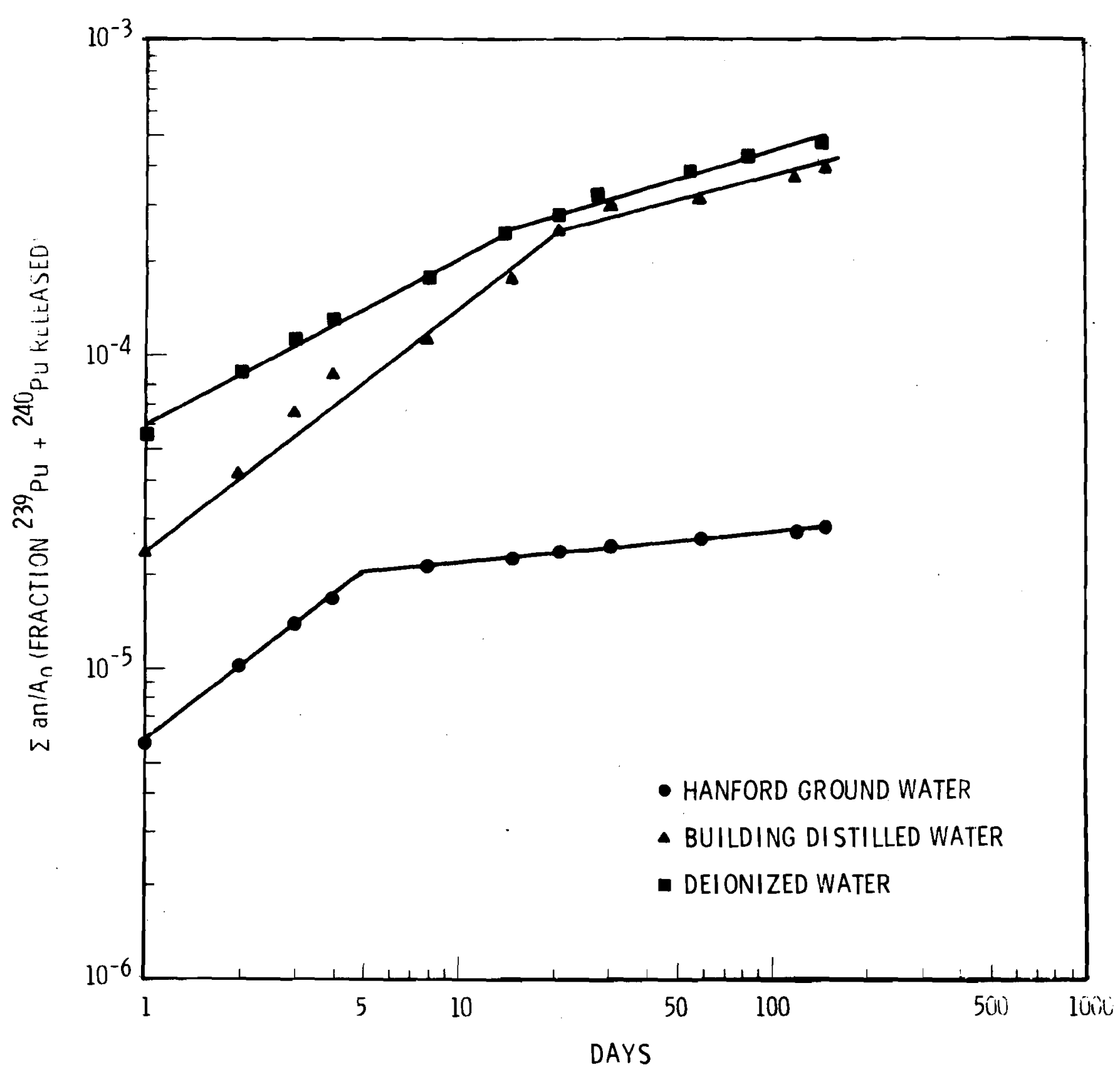

FIGUIRE 5. Fraction of $239 \mathrm{pu}+240 \mathrm{pu}$ Released to Leachant 


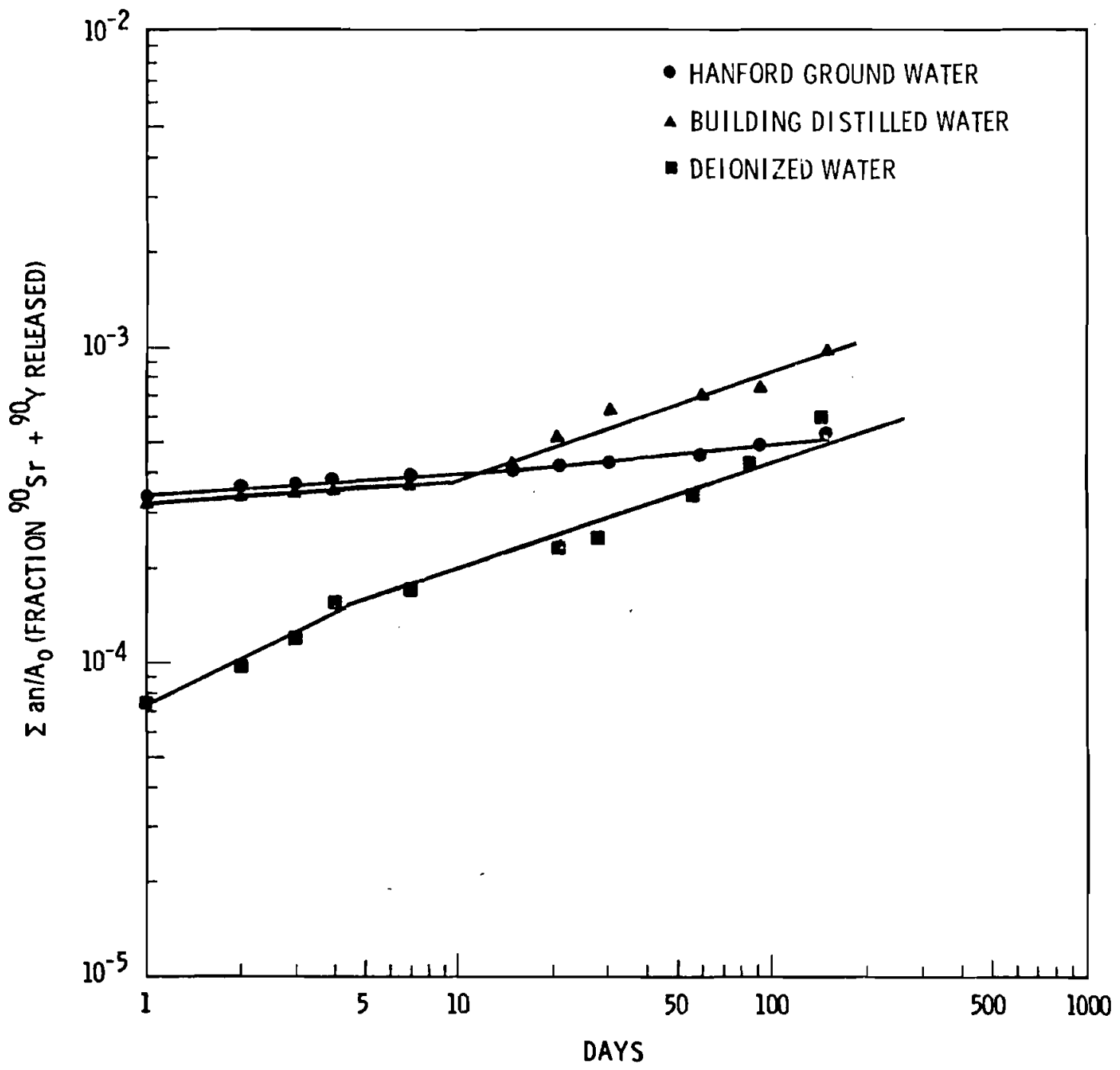

FIGURE 6. Fraction of $90 \mathrm{Sr}+90 \mathrm{Y}$ Released to Leachant 


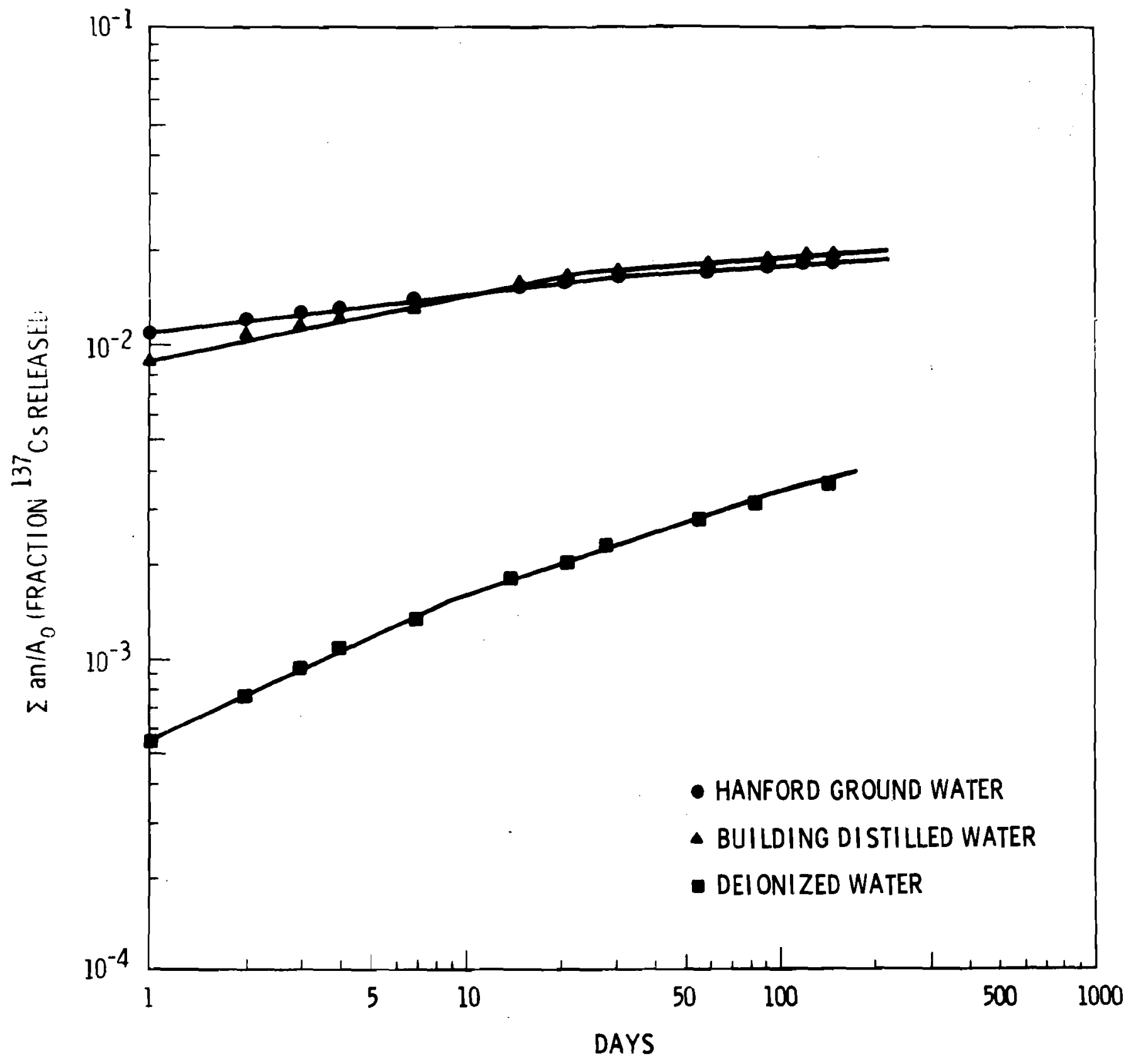

FIGURE 7. Fraction of ${ }^{137}$ CS Released to Leachant 
where $m$ is the slope and $b$ is the intercept. This relationship in the classical form is:

$$
F_{R}=B t^{m}
$$

The values for $m$ are indicative of the leach mechanisms and are tabulated in Table 4. For Hanford ground water the long term leach mechanisms are near identical with an average exponent of $0.07 \pm 0.01$. For deionized water the average value for the exponent is $0.31_{-0.03}^{+n .01}$. The building distilled water exponent values are widespread, 0.35 to $n . n 5$, indicating different leach mechanisms. This is probably attributable to the varying quality of the buildina distilled water, ${ }^{(18)}$ hence varyina ionic concentrations. TABLE 4. Long Term Leach Mechanisms,
exponent $m$

\begin{tabular}{lcccc} 
& $\begin{array}{c}\text { Hanford Ground } \\
\text { Water }\end{array}$ & & $\begin{array}{c}\text { Buildina } \\
\text { nistilled } \\
\text { Water }\end{array}$ & $\begin{array}{c}\text { Deionized } \\
\text { Water }\end{array}$ \\
\cline { 2 - 3 } $\mathrm{Pu}-239+240$ & 0.07 & & 0.26 & 0.30 \\
$\mathrm{Sr}-90+\mathrm{Y}-90$ & 0.07 & & 0.35 & 0.32 \\
$\mathrm{Cm}-244$ & 0.08 & 0.16 & 0.28 \\
$\mathrm{Cs}-137$ & 0.06 & 0.06 & 0.32
\end{tabular}

It is beyond the scope of this report to analyze the leach mechanisms. However the large difference in the exponent values for deionized water and Hanford ground water warrants some discussion. An exnonent of 0.5 would indicate a constant diffusion coefficient for the radioisotopes released. The 0.31 exponent for deionized water probably reflects an inverse relationship of the diffusion coefficient for the radioisotope with its ionic concentration in the leachant. In Hanford around water the exponent of 0.07 reflects the major role of dissolved chemicals in the suppression of leach rate. The varyina exponent for the building distilled water from 0.06 to 0.35 re-emphasizes the need for constant leachant composition during leach testing. Deionized water is a reproducible leachant which gives conservative results and allows for comparison of data with those of other investiqations. 


\section{ACKNOWLEDGMENT}

The author is very arateful to C. E. Bigelow for his assistance in performing the experimental work. The author is also indebted to

J. E. Mendel for many helpful discussions and for reviewing this paper. 


\section{REFERENCES}

1. B. E. Paige, Leachability of Alumina Calcine Produced in the Idaho Was te Calcining Aluminum Waste, In0-14672, Phillips Petroleum Company, Idaho Falls, Idaho, February 1966

2. J. L. McElroy, K. J. Schneider, J. N. Hartley, J. F. Mendel, F. L. Richardson, R. W. McKee and A. G. Blasewitz, Waste Solidification Program Summary Report, Volume II, Evaluation of WSEP Hiqh-Level Waste Solidification Processes, BNWL-1667, Battelle-Northwest, Richland, Washinaton, July 1972.

3. R. E. Routson, Battelle-Northwest, Richland, Washinaton, private communication, August 6, 1975.

4. M. J. Bell, ORIGEN - The ORNL Isotope Generation and nepletion Code, ORNL-4628, Oak Ridge National Laboratory, Oak Ridge, Tennessee, May 1973.

5. F. P. Roberts, Battelle-Northwest, Richland, Washinaton, unpublished data, August 25, 1975.

6. E. D. Jenson, Hanford Engineering Development Laboratory, Westinahouse Hanford Company, Richland, Washington, private communication, May 5, 1976.

7. J. E. Mendel, A Review of Leaching Test Methods and the Leachability of Various Solid Media Containing Radioactive Wastes, B:WL-1765, Batte11e-Northwest, Richland, Washinaton, July 1963.

8. G. L. Tingey and W. D. Felix, "Gas Generation from Calcined Waste", Quarterly Progress Report Research and Development Activities Waste Fixation Program, January through March 1974, BNWL-1826, Battelle-Northwest, Richland, Washinaton, pp. 35-38, April 1074.

9. G. L. Tinqey and W. D. Felix, "Gas Generation from Calcined Waste", Quarterly Progress Report Research and Development Activities Waste Fixation Program, April through June 1974, BNhL-1841, BattelleNorthwest, Richland, Washington, pp. 44-47, July 1974.

10. E. D. Hespe, "Leach Testinq of Immobilized Padioactive Waste Solids-A Proposal for a Standard Method", Atomic Energy Peview, pp. 195-207, Vol. 9, 1971.

11. J. E. Mendel and J. L. McElroy, Waste Solidification Program, Vol. 1n, Evaluation of Solidified Waste Products, BNWL-1666, Battelle, Richland, Washington, July 1972 . 
12. R. Eliassen and M. I. Goldman, Fixation in Vitreous Matrices of High Activity Fission Product Wastes, TID-7613, pp. 576-589, 1961.

13. R. H. Burns, "Solidification of Low- and Intermediate-Level Wastes," Atomic Energy Review, Vol. 9, No. 3, pp. 547-599, 1971.

14. B. E. Paige, Leachability of Alumina Calcine Produced in the Idaho Waste Calcining Facility, IN-1011, Phillips Petroleum Company, IRTS, Idaho Falls, Idaho, July 1966.

15. R. T. Allemann, "Leaching Tests of Spray Calcined Melts," Ouarterly Progress Report, Research and Development Activities, Fixation of Radioactive Residues, 0ctober through December 1964, BattelleNorthwest, Richland, Washinaton, pp. 16-18, January 1975.

16. N. N. Elliot and D. B. Autry, The Durability of "FINGAL" Glass, Part I. Discussion of Method and Effect of Leaching Conditions, AERE-R5151. Atomic Energy Research Estah1ishment, Harwe11, March 1067.

17. W. Heimerl, H. Heine, L. Kahl, H. H. Leoi, W. Latzi, G. Malow, E. Schiener and P. Schubert, Research on Glasses for Fission Product Fixation, Summary Report, January 19ER - June 1971, HMI-B1ก9. Hahn-Meitner Institute, Berlin, September 1971.

18. J. H. Westsik, Jr., Battelle-Northwest, Richland, Washinaton, private communication, November 24, 1975. 
APPENDIX 


\section{LEACH TEST RESULTS}

LEACH TEST IDENTIFICATION NUMBER Z Z-2 .LABORATORY WHERE TESTS PERFORMED Battelle-Northwest

- ANALYST

\section{DATE RESULTS REPORTED}

- Part A. Description of Leach Specimen

Specimen Identification Number Z-2

Proportion of Waste Incorporated in Mixture Irradiated LWR fuel pellet Weight \% or Volume \%

(Based on initial volumes)

Type of Waste, Chemical and Radioisotopic Composition, and Specific Activity of the Waste

Type and Composition of the Immobilization Material

Preparation of Specimen

Storage Conditions

Appearance: 
LEACH TEST RESULTS

Page_of _

LEACH TEST IDENTIFICATION NUMBER Z Z Z-2

LABORATORY WHERE TESTS PERFORMED Battelle-Northwest

ANALYST

DATE RESULTS REPORTED

Part B. Description of Leach Test Procedure

Sample Preparation*

Initial Weight of Sample, W 5.18 .33 arams

Volume of Sample, $V$

Surface Area of Sample, $\mathrm{s} \quad 11.7 \mathrm{~cm}^{2}$

Method of Surface Area Determination genmetric approxirlation

Diagram of Leach Apparatus: Paige type

Leachant Hanford Ground Water

Leachant Volume, $V_{\mathrm{L}}-\frac{500 \mathrm{ml}}{\mathrm{drain}}$

Sampling Procedure _ drain leachant and refill at samplina

Analyical Techniques:

$\mathrm{pH}$ Instrument Identification and Calibration

Constituent $a_{1}$, Analytical Procedure, Standard Deviation of Method.

Constituent $\mathrm{a}_{2}$, Analytical Procedure, Standard Deviation of Method

*If different from "Preparation of Specimen" in Part A. 


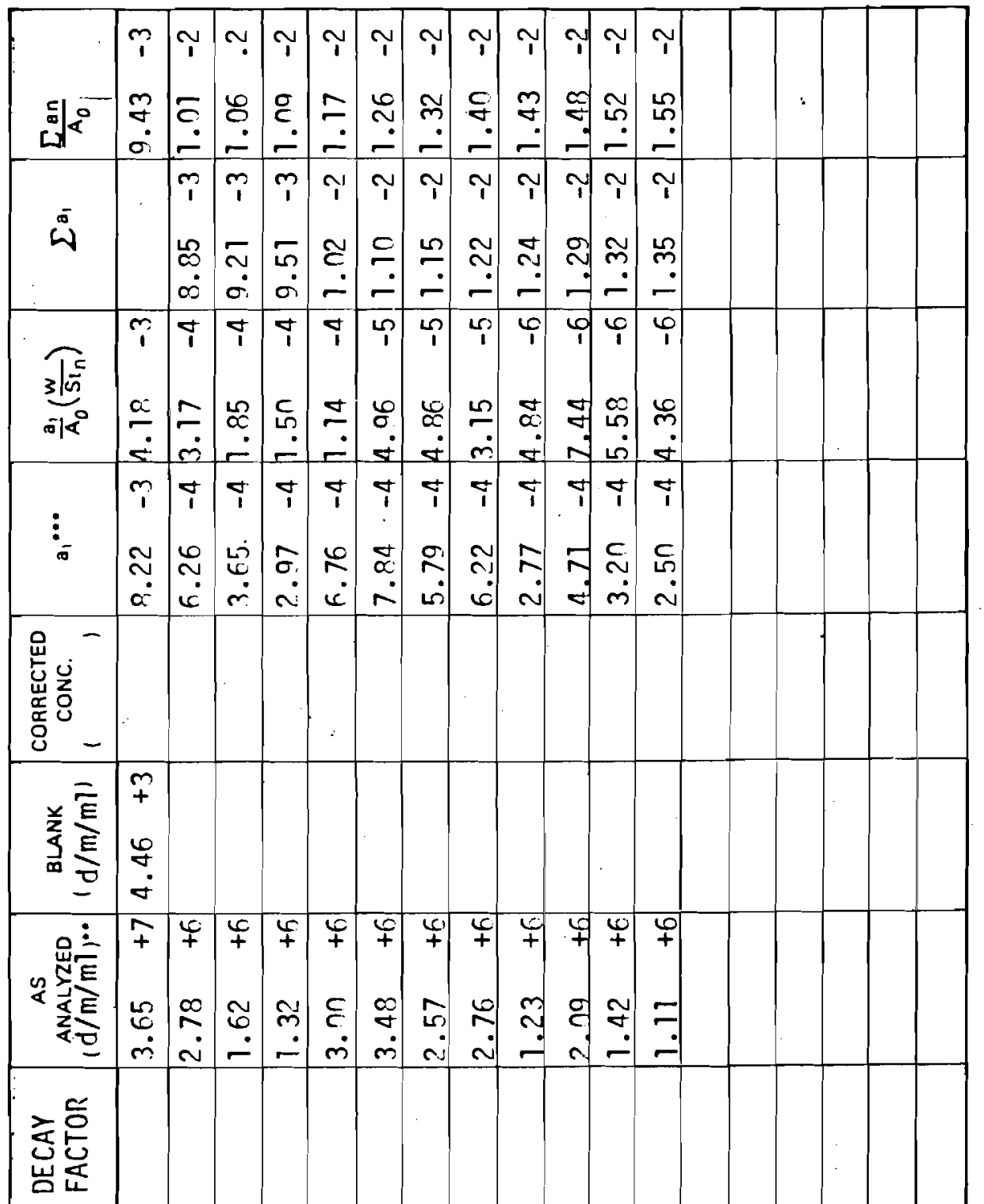

产畜

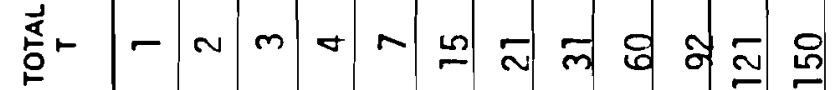


LEACH TEST IDENTIFICATION NUMBER ZZ-2

LABORATORY WHERE TESTS PERFORMED

ANALYST

DATE RESULTS REPORTS

\section{Part C. Experimental Data}

Constituent Analyzed, A Cesium-134

Initial Amount in Sample $A_{0}-1.295 \mathrm{Ci}$

\begin{tabular}{|c|c|c|c|c|c|c|c|c|c|c|c|c|}
\hline \multicolumn{2}{|c|}{ TIME AND DATE } & \multirow{2}{*}{$i_{n}$} & \multirow{2}{*}{$\underset{\mathrm{r}}{\text { TOTAL }}$} & \multirow{2}{*}{$\begin{array}{l}\text { DAYS } \\
\text { DECAY }\end{array}$} & \multirow{2}{*}{$\begin{array}{l}\text { DECAY } \\
\text { FACTOR }\end{array}$} & \multirow{2}{*}{$\begin{array}{c}\text { AS } \\
\text { ANALYZED } \\
1 \mathrm{~d} / \mathrm{m} / \mathrm{ml} \cdots\end{array}$} & \multirow{2}{*}{$\begin{array}{c}\text { BLANK } \\
(\mathrm{d} / \mathrm{m} / \mathrm{m}\rceil\end{array}$} & \multirow{2}{*}{$\mid \begin{array}{c}\text { CORRECTED } \\
\text { CONC. } \\
1\end{array}$} & \multirow{2}{*}{$a_{1} \cdots$} & \multirow{2}{*}{$\frac{a_{1}}{A_{0}}\left(\frac{w}{S_{n}}\right)$} & \multirow{2}{*}{$\sum a_{1}$} & \multirow{2}{*}{$\frac{\sum_{a n} !}{A_{0}}$} \\
\hline IN & OUT & & & & & & & & & & & \\
\hline $8-18-75$ & $8-19-75$ & 1 & 1 & 21 & 0.99 .6 & & & & $9.11-3$ & & & \\
\hline $8-19-75$ & $8-20-75$ & 1 & 2 & 21 & 0.096 & & & & $5.71 \quad-4$ & & & \\
\hline $8-20-75$ & $8-21-75$ & 1 & 3 & 20 & 0.987 & & & & $3.35-4$ & & & \\
\hline $8-21-75$ & $8-22-75$ & 1 & 4 & 19 & 0.037 & & & & $2.76-4$ & & & \\
\hline $8-22-75$ & $8-25-75$ & 3 & 7 & 16 & 0.989 & & & 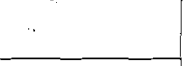 & $6.26 \quad-4$ & & & \\
\hline $8-25-75$ & $9-2-75$ & 8 & 15 & 16 & 0.090 & & & & $7.83 \cdot-4$ & & & \\
\hline $9-2-75$ & $9-8-75$ & 6 & 21 & 18 & 0.998 & & & & $5.03 \quad-4$ & & & \\
\hline $9-8-75$ & $9-18-75$ & 10 & 31 & 44 & 0.971 & & & & $6.45 \quad-4$ & & & \\
\hline $9-18-75$ & $10-17-75$ & 29 & 60 & 16 & 0.980 & & & & $2.79-4$ & & & \\
\hline $10-17-75$ & $11-18-75$ & 32 & 92 & 30 & 0.080 & & & & $4 . n 4 \quad-4$ & & & \\
\hline $11-18-75$ & $12-17-75$ & 29 & 121 & 45 & 0.270 & & & & 2.6n -4 & & & \\
\hline $12-17-75$ & $1-15-76$ & 29 & 150 & 16 & $0.9,99$ & & & & $1.03 \quad-4$ & & & \\
\hline$\lambda=15-76$ & & & & & & & & & & & & \\
\hline & & & & & & & & & & & & \\
\hline & & & & & & & & & & & & \\
\hline & & & & & & & & & & & & \\
\hline & & & & & & & & & & & & \\
\hline & & & & & & & & & & & & \\
\hline
\end{tabular}


.EACH TEST IDENTIFICATION NUMBER

Z-2

ABORATOAY WHERE TESTS PERFORMED

ANALYST

JATTE RESULTS REPORTS

'an C. Experimental Data

Constituent Analyzed, A

PIutonium-239 $+24 n$

Initial Amount in Sample $A_{0}$

4.368 × $\times 10^{-3}-3+21$

\begin{tabular}{|c|c|c|c|c|c|c|c|c|c|c|c|c|c|c|c|c|c|}
\hline \multicolumn{2}{|c|}{ TIME AND DATE- } & \multirow{2}{*}{$i_{n}$} & \multirow{2}{*}{$\underset{T}{\text { TOTAL }}$} & \multirow{2}{*}{$\begin{array}{l}\text { DAYS } \\
\text { DECAY }\end{array}$} & \multirow{2}{*}{$\begin{array}{l}\text { DECAY } \\
\text { FACTOR }\end{array}$} & \multirow{2}{*}{\multicolumn{2}{|c|}{$\begin{array}{c}\text { AS } \\
\text { ANALYZED } \\
(\mathrm{d} / \mathrm{m} / \mathrm{m} 7)^{\circ}\end{array}$}} & \multirow{3}{*}{$1^{\text {BLANK }}$} & \multirow{2}{*}{$\begin{array}{l}\text { CORRECTED } \\
\text { CONC. } \\
1\end{array}$} & \multirow{2}{*}{\multicolumn{2}{|c|}{$a_{1} \cdots$}} & \multirow{2}{*}{\multicolumn{2}{|c|}{$\frac{B_{1}}{A_{0}}\left(\frac{w}{S t_{n}}\right)$}} & \multirow{2}{*}{\multicolumn{2}{|c|}{$\sum a_{1}$}} & \multirow{2}{*}{\multicolumn{2}{|c|}{$\frac{\sum_{\text {an }}^{\prime}}{A_{0}}$}} \\
\hline IN & OUT & & & & & & & & & & & & & & & & \\
\hline $8-18-75$ & $8-19-75$ & 1 & 1 & & & 1.36 & & & 8 & 3.06 & -8 & 3.10 & -6 & 3.06 & -8 & 7.00 & -6 \\
\hline $8-19-75$ & $8-20-75$ & 1 & 2 & & & 7.3 & +1 & & & 1.64 & -8 & 1.66 & -6 & 4.70 & -8 & 1.08 & -5 \\
\hline $8-20-75$ & $8-21-75$ & 1 & 3 & & . & 5.7 & +1 & & & 1.28 & -8 & 1.30 & -6 & 5.98 & -8 & 1.37 & -5 \\
\hline $8-21-75$ & $8-22-75$ & 1. & 4 & & & 5.6 & +1 & & & 1.26 & -8 & 1.28 & -6 & 7.24 & -8 & 1.66 & -5 \\
\hline $8-22-75$ & $8-25-75$ & 3 & 7 & & & 7.4 & +1 & & $\because$ & 1.67 & -8 & 5.62 & -7 & 8.91 & -8 & 2.04 & -5 \\
\hline $8-25-75$ & $9-2-75$ & 8 & 15 & & & 2.08 & +1 & & & 4.68 & -9 & 5.92 & -8 & 9.38 & -8 & 2.14 & -5 \\
\hline $9-2-75$ & $9-8-75$ & 6 & 21 & & & 9.2 & $n$ & & & 2.07 & -9 & 3.50 & -8 & 9.58 & -8 & $2.2 n$ & -5 \\
\hline $9-8-75$ & $9-18-75$ & 10 & 31 & & & 2.4 & +1 & & - & 5.41 & -9 & 5.48 & -8 & 1.01 & -7 & 2.32 & -5 \\
\hline $9-18-75$ & $10-17-75$ & 29 & 60 & & & 2.0 & +1 & & & 4.50 & -9 & 1.57 & -8 & 1.06 & -7 & 2.42 & -5 \\
\hline $10-17-75$ & $11-18-75$ & 32 & 92 & & & 8.2 & & & & 1.85 & -9 & 5.86 & -9 & 1.08 & -7 & 2.47 & -5 \\
\hline $11-18-75$ & $12-17-75$ & $29^{\circ}$ & 121 & & & 2.0 & +1 & & & $4.5 n$ & -9 & 1.57 & -8 & 1.12 & -7 & 2.57 & -5 \\
\hline$-12-17-75$ & $1-15=76$ & 29 & 150 & & & 2.33 & +1 & & & 5.25 & -9 & 1.83 & -8 & 1.18 & -7 & $\begin{array}{r}2.7 n \\
\end{array}$ & -5 \\
\hline $1-15-75$ & & & & & & - & &. & $\ldots \ldots$ & & & & & & & & \\
\hline & $\therefore$ & & & & & & & & & & & & & & & & \\
\hline & & & & $\cdots$ & $\cdots$ & & & & & & & & & & & & \\
\hline & & & & & $\therefore$ & $\ldots$ & & $\ldots$ & & . & & & & & & . & \\
\hline & $\because$ & & & & & & & $\ldots$ & & & & & & & & & \\
\hline & & & & & & & & & & & & & & & & & \\
\hline
\end{tabular}

- Concentration in leachate, show units.

-.. $a_{1}=$ corracted concentration $\times V_{L} \times$ facior to conver to same units as $A_{0}$. 
$\because$

LEACH TEST IDENTIFICATION NUMBER Z-2

LABORATORY WHERE TESTS PERFORMED

ANALYST

DATE RESULTS REPORTS

Pen C. Experimental Data

Constituent Analyzed, A Curium-242

Initial Amount in Sample $A_{0} \quad 1.825 \times 10^{-2} \mathrm{Ci}$

\begin{tabular}{|c|c|c|c|c|c|c|c|c|c|c|c|c|}
\hline \multicolumn{2}{|c|}{ TIME AND DATE- } & \multirow{2}{*}{ in } & \multirow{2}{*}{ TOTAL } & \multirow{2}{*}{$\begin{array}{l}\text { DAYS } \\
\text { DECAY }\end{array}$} & \multirow{2}{*}{$\begin{array}{l}\text { DECAY } \\
\text { FACTOR }\end{array}$} & \multirow{2}{*}{$\begin{array}{c}\text { AS } \\
\text { ANALYZED } \\
\mathrm{ld} / \mathrm{m} / \mathrm{ml} l \cdots\end{array}$} & \multirow{2}{*}{$1^{\text {BLANK }}$} & \multirow{2}{*}{$\begin{array}{l}\text { CORRECTED } \\
\text { CONC. } \\
1\end{array}$} & \multirow{2}{*}{$a_{1} \cdots$} & \multirow{2}{*}{$\frac{a_{1}}{A_{0}}\left(\frac{w}{S s_{n}}\right)$} & \multirow{2}{*}{$\sum a_{1}$} & \multirow{2}{*}{$\frac{\sum_{\text {an }}^{\prime}}{A_{0}}$} \\
\hline IN & OUT & & & & & & & & & & & \\
\hline $8-18-75$ & $8-19-75$ & 1 & 1 & 30 & 0.907 & $4.13+2$ & & & $1.03-7$ & & & \\
\hline $8-19-75$ & $8-20-75$ & 1 & 2 & 29 & 0.011 & $5.1+1$ & & & $1.26 \quad-P$ & & & \\
\hline $8-20-75$ & $8-21-75$ & 1 & 3 & 28 & 0.914 & $5.9+1$ & & & $1.45 \quad-8$ & & & \\
\hline $8-21-75$ & $8-22-75$ & 1. & 4 & 27 & 0.917 & $3.5+1$ & & & $8.6 n \quad-9$ & & & \\
\hline $8-22-75$ & $8-25-75$ & 3 & 7 & 24 & 0.926 & $4.7+1$ & &. & $1.14 \quad-8$ & & & \\
\hline $8-25-75$ & $9-2-75$ & 8 & 15 & 31 & 0.905 & $2 . n 2+1$ & & & $5.03 \cdot-9$ & & & \\
\hline $9-2-75$ & $9-8-75$ & 6 & 21 & 25 & 0.923 & $3.9+1$ & & & $0.52 \quad-9$ & & & \\
\hline $9-8-75$ & $9-18-75$ & 10 & 31 & 26 & $0.92 n$ & $1.7+1$ & & & $4: 16 \quad-9$ & & & \\
\hline $9-18-75$ & $10-17-75$ & 29 & 60 & 10 & 0.942 & $1.1+1$ & & & $2.63 \quad-9$ & & & \\
\hline $10-17-75$ & $11-18-75$ & 32 & 92 & 196 & 0.449 & $3.5 \quad 0$ & & & $1.81-9$ & & & \\
\hline $11-18-75$ & $12-17-75$ & 29 & 121 & 37 & 0.886 & $1.38+1$ & & & $3.51 \quad-9$ & & & \\
\hline $12-17-75$ & $1-15-76$ & 29 & 150 & 8 & 0.975 & $3.39+1$ & & & $7.83 \quad-9$ & & & \\
\hline $1-15-75$ & & & & & & & & & & & & \\
\hline & & & & & & & & & & & & \\
\hline & & & & & & & & & & & & \\
\hline & & & & & & & & & & & & \\
\hline & & & & & & & & & & & & \\
\hline & & & & & & & & & & & & \\
\hline
\end{tabular}


LABORATORY WHERE TESTS PERFORMED

ANALYST

DATE RESULTS REPORTS

\section{Part C. Experimental Data}

Constituent Analyzed, A

Initial Amount in Sample $A_{0}$

Curium-244

$0.028734 \mathrm{Ci}$

\begin{tabular}{|c|c|c|c|c|c|c|c|c|c|c|c|c|c|c|c|c|c|}
\hline \multicolumn{2}{|c|}{ TIME AND DATE } & \multirow{2}{*}{ 'n } & \multirow{2}{*}{$\underset{T}{\text { TOTAL }}$} & \multirow{2}{*}{$\begin{array}{l}\text { DAYS } \\
\text { DECAY }\end{array}$} & \multirow{2}{*}{$\begin{array}{l}\text { DECAY } \\
\text { FACTOR }\end{array}$} & \multirow{2}{*}{\multicolumn{2}{|c|}{$\begin{array}{c}\text { AS } \\
\text { ANALYZED } \\
(\mathrm{d} / \mathrm{m} / \mathrm{ml})^{* *} \\
\end{array}$}} & \multirow{3}{*}{$\frac{\begin{array}{c}\text { BLANK } \\
(\mathrm{d} / \mathrm{m} / \mathrm{m}]^{\prime}\end{array}}{<?}$} & \multirow{2}{*}{$\begin{array}{l}\text { CORAECTED } \\
\text { CONC. } \\
1\end{array}$} & \multirow{2}{*}{\multicolumn{2}{|c|}{$a_{1} \cdots$}} & \multirow{2}{*}{\multicolumn{2}{|c|}{$\frac{a_{1}}{A_{0}}\left(\frac{w}{S i_{n}}\right)$}} & \multirow{2}{*}{\multicolumn{2}{|c|}{$\sum a_{1}$}} & \multirow{2}{*}{\multicolumn{2}{|c|}{$\frac{\sum_{\text {an }}{ }^{\prime}}{A_{0}}$}} \\
\hline IN & our & & & & & & & & & & & & & & & & \\
\hline $8-18-75$ & $8-19-75$ & 1 & 7 & $\cdot$ & & 4.79 & +2 & & $\because$ & $1 . n 6$ & -7 & 1.63 & -6 & 1.06 & -7 & 3.68 & -6 \\
\hline $8-19-75$ & $8-20-75$ & 1 & 2 & & & 4.5 & +1 & & & 1.01 & -9 & 1.56 & -7 & 1.16 & -7 & 4.04 & -6 \\
\hline $8-20-75$ & $8-21-75$ & 1 & 3 & & . & 6.6 & +1 & & & $1.4 n$ & -8 & $? .29$ & -7 & 1.31 & -7 & 4.55 & -6 \\
\hline $8-21-75$ & $8-22-75$ & 1. & 4 & & & 4.1 & +1 & & & 9.23 & -9 & 1.42 & -7 & 1.40 & -7 & 4.88 & -6 \\
\hline $8-22-75$ & $8-25-75$ & 3 & 7 & & & 3.6 & +1 & & $\because$ & 8.11 & -9 & 4.16 & -8 & 1.48 & -7 & 5.16 & -6 \\
\hline $8-25-75$ & $9-2-75$ & 8 & 15 & & & 2.09 & +1 & & & 4.71 & -9 & $9 . n 7$ & -9 & 1.53 & -7 & 5.32 & -6 \\
\hline $9-2-75$ & $9-8-75$ & 6 & 21 & & & 3.1 & +1 & & & 6.93 & -9 & 1.79 & -8 & $1.6 n$ & -7 & 5.56 & -6 \\
\hline $9-8-75$ & $9-18-75$ & 10 & 31 & & & 1.7 & +1 & & $\theta$ & 3.83 & -8 & $5.9 n$ & -9 & 1.54 & -7 & $5.7 n$ & -6 \\
\hline $9-18-75$ & $10-17-75$ & 29 & 60 & & & $2 . n$ & +1 & & & $4.5^{n}$ & $-?$ & 2.39 & -9 & 1.68 & -7 & 5.85 & -6 \\
\hline $10-17-75$ & $11-18-75$ & 32 & 92 & & & 6.1 & $n$ & & & 1.37 & -9 & $6.60-$ & -10 & 1.69 & -7 & 5.80 & -6 \\
\hline $11-18-75$ & $12-17-75$ & 29 & 121 & & & 2.16 & +1 & & & $4.8,6$ & -9 & 2.58 & -9 & 1.74 & -7 & 6.06 & -6 \\
\hline $12-17-75$ & $1-15-76$ & 29 & 150 & & & 1.00 & +1 & & & 1.12 & -8 & 5.07 & -9 & 1.85 & -7 & 6.45 & $-\sigma_{1}$ \\
\hline $1-15-75$ & & & & & & & & & & & & & & & & & \\
\hline & & & & & & & & & & & & & & & & & \\
\hline & & & & & & & & & & & & & & & & & \\
\hline & & & & & & & & & & & & & & & & & \\
\hline & & & & & & & & & & & & & & & & & \\
\hline & & & & & & & & & & & & & & & & & \\
\hline
\end{tabular}

- Concentration in leachate, show units.

$\cdots s_{1}=$ corrected concentration $\times V_{L} \times$ factor to convert to same units as $A_{0}$. 
LEACH TEST IDENTIFICATION NUMBER Z Z-2

LABORATORY WHERE TESTS PERFORMED

ANALYST

DATE RESULTS REPORTS

\section{Pan C. Experimental Date}

Constituent Anolyzed, A Antimony- 125

Initial Amount in Sample $A_{0} \quad \frac{3.684 \times 10^{-2}}{C} \mathrm{Ci}$

\begin{tabular}{|c|c|c|c|c|c|c|c|c|c|c|c|c|c|c|}
\hline \multicolumn{2}{|c|}{ TIME AND DATE } & \multirow{2}{*}{$\ln _{n}$} & \multirow{2}{*}{$\underset{T}{\text { TOTAL }}$} & \multirow{2}{*}{$\begin{array}{l}\text { DAYS } \\
\text { DECAY }\end{array}$} & \multirow{2}{*}{$\begin{array}{l}\text { DECAY } \\
\text { FACTOR }\end{array}$} & \multirow{2}{*}{\multicolumn{2}{|c|}{$\begin{array}{c}\text { AS } \\
\text { ANALYZED } \\
1 \mathrm{~d} / \mathrm{m} / \mathrm{m}, \ldots\end{array}$}} & \multirow{2}{*}{$1^{\text {BLANK }}$} & \multirow{2}{*}{$\begin{array}{l}\text { CORRECTED } \\
\text { CONC. } \\
1\end{array}$} & \multirow{2}{*}{\multicolumn{2}{|c|}{$a_{1} \cdots$}} & \multirow{2}{*}{$\frac{a_{1}}{A_{0}}\left(\frac{w}{S s_{n}}\right)$} & \multirow{2}{*}{$\sum a_{1}$} & \multirow{2}{*}{$\frac{F_{\text {an }}}{A_{0}}$} \\
\hline $\mathbb{I N}$ & our & & & & & & & & & & & & & \\
\hline $8-18-75$ & $8-19-75$ & 1 & 1 & 164 & 0.917 & 2.85 & +4 & & & 7.02 & -6 & & & \\
\hline $8-19-75$ & $8-20-75$ & $\overline{1}$ & 2 & 163 & 0.917 & 8.87 & +3 & & & 2.18 & -6 & & & \\
\hline $8-20-75$ & $8-21-75$ & 1 & 3 & 162 & 0.918 & 6.52 & +3 & & & 1.60 & & & & \\
\hline $8-21-75$ & $8-22-75$ & 1 & 4 & 161 & 0.918 & 6.71 & +3 & & & 1.65 & -6 & & & \\
\hline $8-22-75$ & $8-25-75$ & 3 & 7 & 158 & 0.920 & 1.27 & +4 & & . & 3.11 & -6 & & & \\
\hline $8-25-75$ & $9-2-75$ & 8 & 15 & 150 & 0.924 & 1.38 & +4 & & & 3.36 & -6 & & & \\
\hline $9-2-75$ & $9-8-75$ & 6 & 21 & 246 & 0.875 & 8.4 & +3 & & & 2.16 & -6 & & & \\
\hline $9-8-75$ & $9-18-75$ & 10 & 31 & 237 & 0.880 & 6.79 & +3 & & & 1.74 & -6 & & & \\
\hline $9-18-75$ & $10-17-75$ & 29 & 60 & 209 & 0.894 & 7.05 & +3 & & & 1.78 & -6 & & & \\
\hline $10-17-75$ & $11-18-75$ & 32 & 92 & 177 & 0.910 & 1.24 & +4 & & & 3.07 & -6 & & & \\
\hline $11-18-75$ & $12-17-75$ & 29 & 121 & 50 & 0.975 & 1.08 & +4 & & & 2.49 & -6 & & & \\
\hline $12-17-75$ & 1.15 .76 & 29 & 150 & 22 & 0.989 & 1.06 & +4 & & & 2.41 & -6 & & & \\
\hline & & & & & & & & & & & & & & \\
\hline & & & & & & & & & & & & & & \\
\hline & & & & & & & & & & & & & & \\
\hline & & & & & & & & & & & & & & \\
\hline & & & & & & & & & & & & & & \\
\hline
\end{tabular}

- Concentration in leachsto, show units.

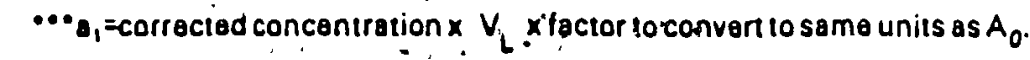


LEACH TEST IDENTIFICATION NUMBEF

LABORATORY WHERE TESTS PERFORMED

ANALYST

DATE RESULTS REPORTS

Part C. Experimental Data

Constituent Analyzed, A

Europi um- 154

Initial Amount in Sample $A_{0}$

0.062968 ri

\begin{tabular}{|c|c|c|c|c|c|c|c|c|c|c|c|c|c|c|}
\hline \multicolumn{2}{|c|}{ TIME AND DATE } & \multirow{2}{*}{$I_{n}$} & \multirow{2}{*}{$\begin{array}{c}\text { TOTAL } \\
\text { T }\end{array}$} & \multirow{2}{*}{$\begin{array}{l}\text { DAYS } \\
\text { DECAY }\end{array}$} & \multirow{2}{*}{$\begin{array}{l}\text { DECAY } \\
\text { FACTOR }\end{array}$} & \multirow{2}{*}{\multicolumn{2}{|c|}{$\begin{array}{c}\text { AS } \\
\text { ANALYZED } \\
(\mathrm{d} / \mathrm{m} / \mathrm{m}) \cdot \cdot\end{array}$}} & \multirow{3}{*}{$1^{\text {BLANK }}$} & \multirow{2}{*}{$\begin{array}{c}\text { CORRECTED } \\
\text { CONC. }\end{array}$} & \multirow{2}{*}{$a_{1} \cdots$} & \multirow{2}{*}{\multicolumn{2}{|c|}{$\frac{a_{1}}{A_{0}}\left(\frac{w}{S I_{n}}\right)$}} & \multirow{2}{*}{$\sum a_{1}$} & \multirow{2}{*}{$\frac{\sum \text { an }}{A_{0}}$} \\
\hline IN & OUT & & & & & & & & & & & & & \\
\hline $8-18-75$ & $8-19-75$ & 1 & 1 & & & 4.5 & $+?$ & & & $1 . n 1-7$ & 7.11 & -7 & $1.01-7$ & $1.61-6$ \\
\hline $8-19-75$ & $8-2 \theta-75$ & 1 & 2 & & & $? .9$ & $+?$ & & & $5.53-9$ & $0.5^{\circ}$ & -7 & $1.67 \quad-7$ & $2.65-6$ \\
\hline $8-20-75$ & $8-21-75$ & 1 & 3 & & & $? .1$ & $+?$ & & & $0.73 .-3$ & $3.3^{3}$ & -7 & $\begin{array}{ll}2.11 & -7\end{array}$ & $3.40-6$ \\
\hline $8-21-75$ & $8-22-75$ & 1 & 4 & & & 1.4 & $+?$ & & & $3.15-?$ & 2.21 & -7 & $2.16 \quad-7$ & $3.0 n-6$ \\
\hline $8-22-75$ & $8-25-75$ & 3 & 7 & & & 3.3 & $+?$ & & $\because$ & $7.43 \quad-?$ & 1.74 & -7 & $3.2 \cap \quad-7$ & $5.78 \quad-6$ \\
\hline $8-25-75$ & $9-2-75$ & 8 & 15 & & & & & & & & & & & \\
\hline $9-2-75$ & $9-8-75$ & 6 & 21 & & & & & & & & & & & \\
\hline $9-8-75$ & $9-18-75$ & 10 & 31 & & & 1.7 & +2 & & & & & & & \\
\hline $9-18-75$ & $10-17-75$ & 29 & 60 & & & $<1$ & +2 & & & & & & & \\
\hline $10-17-75$ & $17-18-75$ & 32 & 92 & & & $<1$ & +2 & & & & & & & \\
\hline $11-18-75$ & $12-17-75$ & 29 & 127 & & & $<1$ & $+?$ & & & & 5.45 & & & \\
\hline $12-17-75$ & $1-15-76$ & 29 & 150 & & & 3.1 & $+?$ & & & & $1 . r 0$ & & & \\
\hline & & & & & & & & & & & & & & \\
\hline & & & & & & & & & & & & & & \\
\hline & & & & & & & & & & & & & & \\
\hline & & & & & & & & & & & & & & \\
\hline & & & & & & & & & & & & & & \\
\hline & & & & & & & & & & & & & & \\
\hline
\end{tabular}

\section{- Concentration in laschate, show units.}

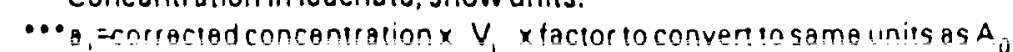


$\because$

LEACH TEST IDENTIFICATION NUMBER

$Z-2$

LABORATORY WHERE TESTS PERFORMED

ANALYST .

DATE RESULTS REPORTS

Part C. Experimental Dato

Constituent Analyzed, A

Ruthen ium-176

Initial Amount in Sample $A_{0}$

$0.8969 \mathrm{Ci}$

\begin{tabular}{|c|c|c|c|c|c|c|c|c|c|c|c|c|c|}
\hline \multicolumn{2}{|c|}{ TIME AND DATE } & \multirow{2}{*}{$t_{n}$} & \multirow{2}{*}{$\underset{T}{\text { TOTAL }}$} & \multirow{2}{*}{$\begin{array}{l}\text { DAYS } \\
\text { DECAY }\end{array}$} & \multirow{2}{*}{$\begin{array}{l}\text { DECAY } \\
\text { FACTOR }\end{array}$} & \multirow{2}{*}{ 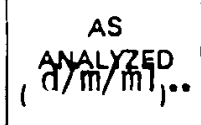 } & \multirow{2}{*}{$1^{\text {BLANK }}$} & \multirow{2}{*}{$\begin{array}{l}\text { CORAECTED } \\
\text { CONC. } \\
1\end{array}$} & \multirow{2}{*}{\multicolumn{2}{|c|}{$a_{1} \cdots$}} & \multirow{2}{*}{$\frac{a_{1}}{A_{0}}\left(\frac{w}{S i_{n}}\right)$} & \multirow{2}{*}{$\sum a_{1}$} & \multirow{2}{*}{$\frac{E_{\text {an }}}{A_{0}}$} \\
\hline IN & OUT & & & & & & & & & & & & \\
\hline $8-18-75$ & $8-19-75$ & 1 & 1 & 164 & 0.778 & $3.71+4$ & & & 1.07 & -5 & & & \\
\hline $8-19-75$ & $8-20-75$ & 1 & 2 & 163 & 0.778 & $7.75+3$ & & & 2.24 & -6 & & & \\
\hline $8-20-75$ & $8-21-75$ & 1 & 3 & 162 & 0.779 & $5.18+3$ & & & 1.50 & & & & \\
\hline $8-21-75$ & $8-22-75$ & 1. & 4 & 161 & 0.781 & $4.23+3$ & & & 1.22 & & & & \\
\hline $8-22-75$ & $8-25-75$ & 3 & 7 & 158 & 0.785 & $5.71+3$ & & . & 1.64 & -6 & & & \\
\hline $8-25-75$ & $9-2-75$ & 8 & 15 & 150 & 0.796 & $3.98+3$ & & & 1.13 & -6 & & & \\
\hline $9-2-75$ & $9-8-75$ & 6 & 21 & 246 & 0.665 & $k 3.25+3$ & & & +1.10 & -6 & & & \\
\hline $9-8-75$ & $9-18-75$ & 10 & 31 & 237 & 0.677 & $3.16+3$ & & & 1.05 & -6 & & & \\
\hline $9-18-75$ & $10-17-75$ & 29 & 60 & 209 & 0.715 & $66.07+2$ & & & +1.91 & -7 & & & \\
\hline $10-17-75$ & $11-18-75$ & 32 & 92 & 177 & 0.759 & $\times 1.13+3$ & & & 3.35 & -7 & & & \\
\hline $11-18-75$ & $12-17-75$ & 29 & 121 & 50 & 0.932 & $2.08+3$ & & & $5 . \cap 3$ & -7 & & & \\
\hline $12-17-75$ & $1-15-76$ & 29 & 150 & 22 & 0.970 & $2.12+3$ & & & 4.92 & -7 & & & \\
\hline & & & & & & & & & & & & & \\
\hline & & & & & & & & & & & & & \\
\hline & & & & & & & & & & & & & \\
\hline & & & & & & & & & & & & & \\
\hline & & & & & & & & & & & & & \\
\hline
\end{tabular}

- Concentration in leachate, show units.

-... $a_{1}=$ correcied concentration $x V_{L}$. tactor to canvert 10 same units as $A_{0}$. 
LEACH TEST IDENTIFICATION NUMBER Z-2 -LABORATORY WHERE TESTS PERFORMED ANALYST

DATE RESULTS REPORTS

\section{Pant C. Exporimental Data}

Constituent Analyzed, A $\longrightarrow$ Strontium-an + Yttrium-an

Initial Amount in Sample $A_{0}$

\begin{tabular}{|c|c|c|c|c|c|c|c|c|c|c|c|c|c|c|c|c|c|}
\hline \multicolumn{2}{|c|}{ TIME AND DATE } & \multirow{2}{*}{$i_{n}$} & \multirow{2}{*}{$\underset{T}{\text { TOTAL }}$} & \multirow{2}{*}{$\begin{array}{l}\text { DAYS } \\
\text { DECAY }\end{array}$} & \multirow{2}{*}{$\begin{array}{l}\text { DECAY } \\
\text { FACTOR }\end{array}$} & \multirow{2}{*}{\multicolumn{2}{|c|}{$\begin{array}{c}\text { AS } \\
\text { ANALYZED } \\
(\mathrm{d} / \mathrm{m} / \mathrm{m} 1) \cdot\end{array}$}} & \multirow{3}{*}{$1^{\text {BLANK }}$} & \multirow{2}{*}{$\begin{array}{l}\text { CORRECTED } \\
\text { CONC. }\end{array}$} & \multirow{2}{*}{\multicolumn{2}{|c|}{$a_{1} \cdots$}} & \multirow{2}{*}{\multicolumn{2}{|c|}{$\frac{a_{1}}{A_{0}}\left(\frac{w}{S ! n}\right)$}} & \multirow{2}{*}{\multicolumn{2}{|c|}{$\sum a_{1}$}} & \multirow{2}{*}{\multicolumn{2}{|c|}{$\frac{\sum_{\text {an }} !}{A_{0}}$}} \\
\hline $\mathbb{I N}$ & OUT & & & & & & & & & & & & & & & & \\
\hline $8-18-75$ & $8-19-75$ & 1 & $T$ & . & & $2 . n$ & $+f$ & & $\because$ & $4.5^{n}$ & -4 & 1.10 & -4 & $4.5 n$ & -4 & 3.36 & -4 \\
\hline $8-19-75$ & $8-20-75$ & 1 & 2 & & & 1.4 & +5 & & & 3.15 & -5 & $1 . \cap 4$ & -5 & $4.9 ?$ & -4 & $3.6 n$ & -4 \\
\hline $8-20-75$ & $8-21-75$ & 1 & 3 & & & 5.1 & +1 & & & 1.92 & -5 & $n . n ?$ & -6 & 4.94 & -4 & 3.60 & -4 \\
\hline $8-21-75$ & $8-22-75$ & 1 & 4 & & & s.n & +1 & & & $1.3 n$ & -5 & 5.05 & $-f$ & 5.12 & -4 & $3.0,3$ & -4 \\
\hline $8-22-75$ & $8-25-75$ & 3 & 7 & & & $7 . ?$ & +4 & & . & $1.6 ?$ & -5 & $1.7^{n}$ & -6 & $5.2 ?$ & -1 & 3.95 & -4 \\
\hline $8-25-75$ & $9-2-75$ & 8 & 15 & & & 6.9 & +1 & & & 1.55 & -5 & $6.1 ?$ & -7 & 5.14 & -1 & $4 . n 6$ & -4 \\
\hline $9-2-75$ & $9-8-75$ & 6 & 21 & & & 6.0 & $+n$ & & & 1.55 & -5 & 3.57 & -7 & 5.50 & -4 & 4.13 & -4 \\
\hline $9-8-75$ & $9-18-75$ & 10 & 31 & & & 7.9 & +1 & & 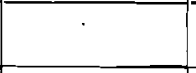 & 1.76 & -5 & 5.91 & -7 & 5.77 & -4 & 4.31 & -4 \\
\hline $9-18-75$ & $10-17-75$ & 29 & 60 & & & 3.8 & $+n$ & & & $1 . n$ & -5 & $? .26$ & -7 & 5.07 & -4 & 4.46 & -4 \\
\hline $10-17-75$ & $11-18-75$ & 32 & 92 & & & 2.1 & +5 & & & 1.73 & -5 & 4.90 & -7 & $F .44$ & -4 & 4.91 & -4 \\
\hline $11-18-75$ & $12-17-75$ & 29 & 121 & & & 1.66 & +5 & & & 3.74 & -5 & 4.27 & -7 & 6.81 & -4 & 5.09 & -4 \\
\hline $12-17-75$ & $1-15-76$ & 29 & 150 & & & 1.7 & +5 & & & $3.3,3$ & -5 & 4.37 & -7 & 7.20 & -4 & 5.39 & -4 \\
\hline \multicolumn{18}{|c|}{$1-15-75$} \\
\hline & & & & & & & & & & & & & & & & & \\
\hline & & & & & & & & & & & & & & & & & \\
\hline & & & & & & & & & & & & & & & & & \\
\hline & & & & & & & & & & & & & & & & & \\
\hline & & & & & & & & & & & & $\cdot$ & & & & & \\
\hline
\end{tabular}




\section{LEACH TEST RESULTS}

LEACH TEST IDENTIFICATION NUMBER _ _ Z Z - 3

LABORATORY WHERE TESTS PERFORMED Battelle-Northwest

ANALYST

DATE RESULTS REPORTED

\section{Part A. Description of Leach Specimen}

Specimen Identification Number ___ Z Z Z

Proportion of Waste Incorporated in Mixture_Irradiated LWR fuel pellet_Weight \%

or

Volume\%

(Based on initial volumes)

Type of Waste, Chemical and Radioisotopic Composition, and Specific Activity of the Waste

Type and Composition of the Immobilization Material

Preparaiton of Specimen

Storage Conditions

Appearance: 
LEACH TEST RESULTS

Page_ol

LEACH TEST IDENTIFICATION NUMBER _ Z-3

LABORATORY WHERE TESTS PERFORMED Battelle-Northwest

ANALYST

DATE RESULTS REPORTED

Part B. Description of Leach Test Procedure

Sample Preparation*

Initial Weight of Sample, $W$

$5.0482 \mathrm{gms}$

Volume of Sample, $\mathrm{V}$

Surface Area of Sample, $\mathbf{S}$

$11.4 \mathrm{~cm}^{2}$

Method of Surface Area Determination

geometric approximation

Diagram of Leach Apparatus:

Paige type

Leachant

Hanford Ground Water

Leachant Volume, $V_{1}$

$500 \mathrm{ml}$

Sampling Procedure

Drain leachant and refill at sampling.

Analytical Techniques:

$\mathrm{pH}$ Instrument Identification and Calibration

Constituent $a_{1}$, Analytical Procedure, Standard Deviation of Method

Constituent $\mathrm{a}_{2}$. Analytical Procedure. Standard Deviation of Method

"If different from "Preparation of Specimen" in Part A. 

Z-3

LEACH TEST IDENTIFICATION NUMBER

\section{LABORATORY WHERE TESTS PERFORMED}

\section{ANALYST}

DATE RESULTS REPORTS

\section{Pan C. Exporimental Data}

Constituent Analyzed, A

Initial Amount in Sample $A_{0}$

Cesium 137

$0.8489 \mathrm{Ci}$

\begin{tabular}{|c|c|c|c|c|c|c|c|c|c|c|c|c|}
\hline \multicolumn{2}{|c|}{ TIME AND DATE } & \multirow{2}{*}{$\ln$} & \multirow{2}{*}{$\underset{\mathrm{r}}{\text { TOrAL }}$} & \multirow{2}{*}{$\begin{array}{l}\text { DAYS } \\
\text { DECAY }\end{array}$} & \multirow{2}{*}{$\begin{array}{l}\text { DECAY } \\
\text { FACTOR }\end{array}$} & \multirow{2}{*}{$\begin{array}{c}\text { AS } \\
\text { ANALYZED } \\
(\mathrm{d} / \mathrm{m} / \mathrm{ml})^{* *} \\
\end{array}$} & \multirow{2}{*}{$\begin{array}{c}\text { BLANK } \\
\mathrm{d} / \mathrm{m} / \mathrm{ml}\end{array}$} & \multirow{2}{*}{$\begin{array}{c}\text { CORRECTED } \\
\text { CONC. } \\
1\end{array}$} & \multirow{2}{*}{$a_{1} \cdots$} & \multirow{2}{*}{$\frac{a_{1}}{A_{0}}\left(\frac{w}{S_{1}}\right)$} & \multirow{2}{*}{$\sum a_{1}$} & \multirow{2}{*}{$\frac{\sum_{\text {an }}{ }^{\prime}}{A_{0}}$} \\
\hline IN & OuT & & & & & & & & & & & \\
\hline $8-18-75$ & $8-19-75$ & 1 & 1 & & & $4.69+7$ & $4.53+3$ & & $1.06-2$ & $5.53-3$ & $1.06-2$ & $1.25-2$ \\
\hline $8-19-75$ & $8-20-75$ & 1 & 2 & & & $5.64 \quad 6$ & & & $1.27-3$ & $6.66-4$ & $1.19-2$ & $1.40-2$ \\
\hline $8-20-75$ & $8-21-75$ & 1 & 3 & & & $2.40+6$ & & & $5.41 \cdot-4$ & $2.83-4$ & $1.24-2$ & $1.46-2$ \\
\hline $8-21-75$ & $8-22-75$ & 1 & 4 & & & $1.66+6$ & & & $3.74-4$ & $1.96-4$ & $1.28-2$ & $1.51-2$ \\
\hline $8-22-75$ & $8-25-75$ & 3 & 7 & & & $3.27+6$ & & $\therefore$ & $7.36-4$ & $1.29-4$ & $1.35-2$ & $1.59-2$ \\
\hline $8-25-75$ & $9-02-75$ & 8 & 15 & & & $5.24+6$ & & & $1.18+3$ & $7.73-5$ & $1.47-2$ & $1.73-2$ \\
\hline $9-02-75$ & $9-08-75$ & 6 & 27 & & & $3.27+6$ & & & $7.36-4$ & $6.43-5$ & $1.54-2$ & $1.82-2$ \\
\hline $9-08-75$ & $9-18-75$ & 10 & 3] & & & $2.50+6$ & & & $5.63-4$ & $2.95-5$ & $1.60-2$ & $1.88-2$ \\
\hline $9-18-75$ & $10-17-75$ & 29 & 60 & & & $3.37+6$ & & & $7.59-4$ & $1.37-5$ & $1.68-2$ & $1.97-2$ \\
\hline $10-17-75$ & $11-18-75$ & 32 & 92 & & & $1.99+6$ & & & $4.48-4$ & $7.34-6$ & $1.72-2$ & $2.03-2$ \\
\hline $11-18-75$ & $12-17-75$ & 29 & 121 & & & $1.47+6$ & & & $3.31-4$ & $5.98-6$ & $1.75-2$ & $2.07-2$ \\
\hline $12-17-75$ & $1-15-76$ & 29 & 150 & & & $1.17+6$ & & & $2.64-4$ & $4.76-6$ & $1.78-2$ & $2.10-2$ \\
\hline & & & & & & & & & & & & \\
\hline & & & & & & & & & & & & \\
\hline & & & & & & & & & & & & \\
\hline & & & & & & & & & & & & \\
\hline & & . & & & & & & & & & & \\
\hline & & & & & & & & & & & & \\
\hline
\end{tabular}


LEACH TEST IDENTIFICATION NUMBER

LABORATORY WHERE TESTS PERFORMED

ANALYST

DATE RESULTS REPORTS

Part C. Experimental Data

Constituent Analyzed, A $\quad$ Cesium-134

Initial Amount in Sample $A_{0}-1.2673 \mathrm{Ci}$

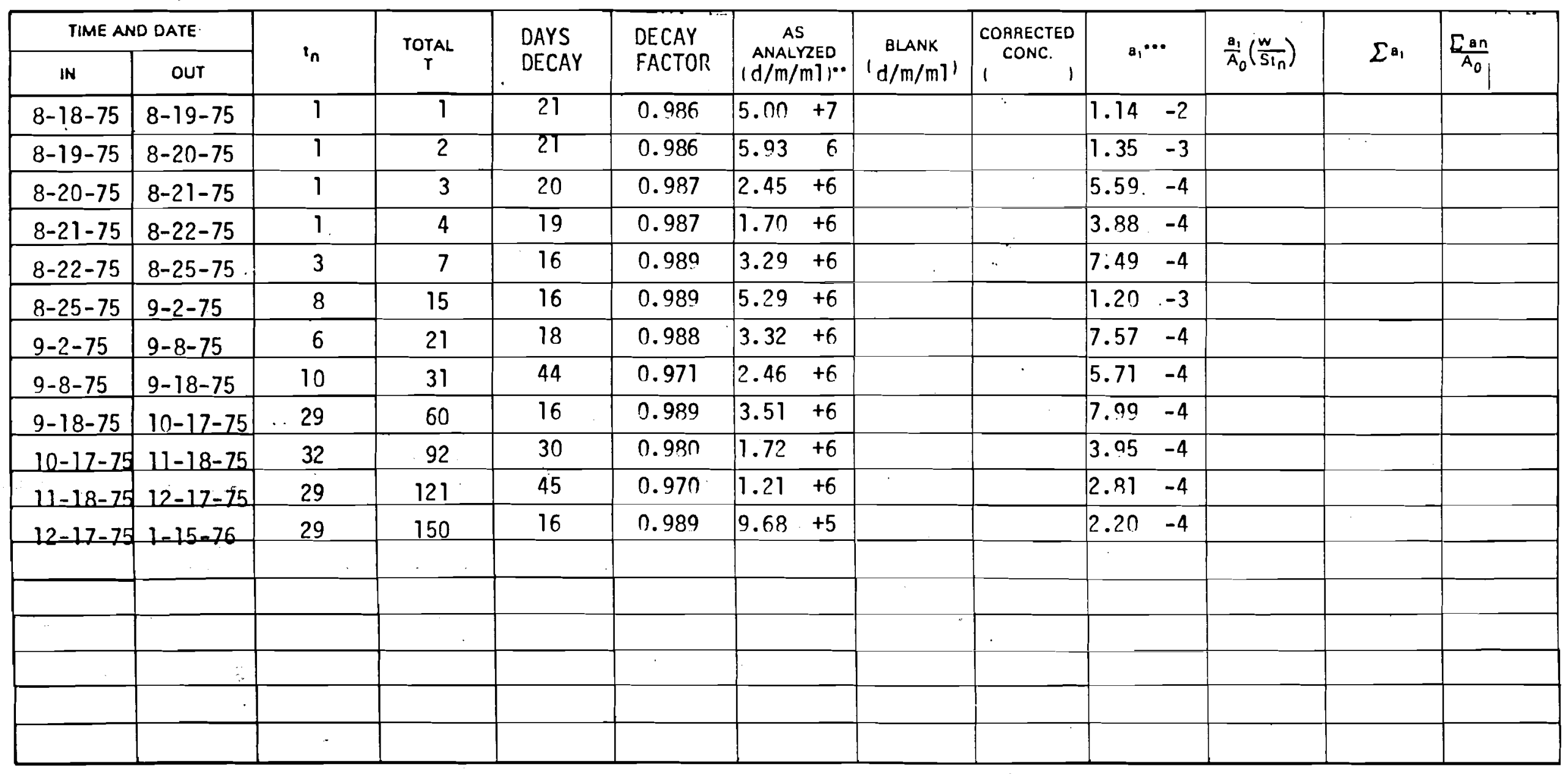

- Concentration in leachate, show units.

$\cdots a_{1}=$ correcied concentration $\times V_{l} \times$ factor to convert to same units as $A_{0}$. 
LABORATORY WHERE TESTS PERFORMED

\section{ANALYST}

DATE RESULTS REPORTS

\section{Part C. Exporimental Datn}

Constituent Analyzed, A

Plutonium $239+240$

Initial Amount in Sample $A_{0}$ $4.2542 \times 10^{-3} \mathrm{Ci}$

\begin{tabular}{|c|c|c|c|c|c|c|c|c|c|c|c|c|}
\hline \multicolumn{2}{|c|}{ TIME AND DATE } & \multirow{2}{*}{ In } & \multirow{2}{*}{$\underset{T}{\text { TOTAL }}$} & \multirow{2}{*}{$\begin{array}{l}\text { DAYS } \\
\text { DECAY }\end{array}$} & \multirow{2}{*}{$\begin{array}{l}\text { DECAY } \\
\text { FACTOR }\end{array}$} & \multirow{2}{*}{$\begin{array}{c}\text { AS } \\
\text { ANALYZED } \\
(\mathrm{d} / \mathrm{m} / \mathrm{ml})^{\cdots}\end{array}$} & \multirow{2}{*}{$\begin{array}{c}\text { BLANK } \\
\mathrm{b} / \mathrm{m} / \mathrm{ml}\end{array}$} & \multirow{2}{*}{$\begin{array}{l}\text { CORRECTEO } \\
\text { CONC. } \\
1\end{array}$} & \multirow{2}{*}{$a_{1} \cdots$} & \multirow{2}{*}{$\frac{B_{1}}{A_{0}}\left(\frac{w}{S_{n}}\right)$} & \multirow{2}{*}{$\sum a_{1}$} & \multirow{2}{*}{$\frac{\sum_{\text {an }}}{A_{0}}$} \\
\hline $\mathbb{I N}$ & OUT & & & & & & & & & & & \\
\hline $8-18-75$ & $8-19-75$ & 1 & 1 & & & $8.3+1$ & $<2$ & 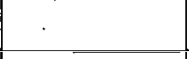 & $1.87-8$ & $1.94-6$ & $1.87-8$ & $4.40-6$ \\
\hline $8-19-75$ & $8-20-75$ & 1 & 2 & & & $9.9+1$ & & & $2.23-8$ & $2.32-6$ & $4.10-8$ & $9.65-6$ \\
\hline $8-20-75$ & $8-21-75$ & 1 & 3 & & . & $8.2+1$ & & & $1.85-8$ & $1.02-6$ & $5.95-8$ & $1.40-5$ \\
\hline $8-21-75$ & $8-22-75$ & 1 & 4 & & & $4.9+1$ & & & $1.10-8$ & $1.15-6$ & $7.05-8$ & $1.66-5$ \\
\hline $8-22-75$ & $8-25-75$ & 3 & 7 & & & $9.1+1$ & & * & $2.05-8$ & $2.12-6$ & $9.10-8$ & $2.14-5$ \\
\hline $8-25-75$ & $9-02-75$ & 8 & 15 & & & $2.7+1$ & & & $6.08-9$ & $7.90-8$ & $9.71-8$ & $2.28-5$ \\
\hline $9-02-75$ & $9-08-75$ & 6 & 21 & & & $3.6+1$ & & & $8.11-9$ & $1.40-7$ & $1.05-7$ & $2.47-5$ \\
\hline $9-08-75$ & $9-18-75$ & 10 & 31 & & & $1.51+1$ & & & $3.40-9$ & $3.52-8$ & $1.09-7$ & $2.55-5$ \\
\hline $9-18-75$ & $10-17-75$ & $\therefore \quad 29$ & 60 & & & $2.2+1$ & & & $4.96-9$ & $1.78-8$ & $1.13-7$ & $2.67-5$ \\
\hline $10-17-75$ & $11-18-75$ & 32 & 92 & & & $8.5 \quad 0$ & & & $1.91-9$ & $6.21 \quad-9$ & $1.14-7$ & $2.70-5$ \\
\hline $11-18-75$ & $12-17-75$ & 29 & 121 & & & $<10$ & & & $(2.25-9)$ & $(8.08--9)$ & $1.17-7$ & $2.75-5$ \\
\hline $12-17-75$ & $1-15-76$ & 29 & 150 & & & $<10$ & & & $2.25-9)$ & $(8.08-9)$ & $11.19-7$ & $2.8]-5$ \\
\hline & & & & & & & & & & & & \\
\hline & & & & & & & & & & & & \\
\hline & & & & & & & & & . & & & \\
\hline & & . & & & & & & & & & & \\
\hline & & & & & & & & & & & & \\
\hline
\end{tabular}


LABORATORY WHERE TESTS PERFORMED

ANALYST

DATE RESULTS REPORTS

\section{Part C. Experimental Data}

Constituent Analyzed, A

Initial Amount in Sample $A_{0}$

Curium 242

$1.777 \times 10^{-2} \mathrm{Ci}$

\begin{tabular}{|c|c|c|c|c|c|c|c|c|c|c|c|c|}
\hline \multicolumn{2}{|c|}{ TIME AND DATE } & \multirow{2}{*}{$i_{n}$} & \multirow{2}{*}{$\begin{array}{c}\text { TOTAL } \\
T\end{array}$} & \multirow{2}{*}{$\begin{array}{l}\text { DAYS } \\
\text { DECAY }\end{array}$} & \multirow{2}{*}{$\begin{array}{l}\text { DECAY } \\
\text { FACTOR }\end{array}$} & \multirow{2}{*}{$\begin{array}{c}\text { AS } \\
\text { ANALYZED } \\
1 \mathrm{~d} / \mathrm{m} / \mathrm{m} \mathrm{I}^{*} \\
\end{array}$} & \multirow{2}{*}{$\begin{array}{c}\text { BLANK } \\
\mathrm{d} / \mathrm{m} / \mathrm{ml}\end{array}$} & \multirow{2}{*}{$\begin{array}{c}\text { CORRECTED } \\
\text { CONC. } \\
1\end{array}$} & \multirow{2}{*}{$a_{1} \cdots$} & \multirow{2}{*}{$\frac{a_{1}}{A_{0}}\left(\frac{w}{S I_{n}}\right)$} & \multirow{2}{*}{$\sum a_{1}$} & \multirow{2}{*}{$\frac{\sum_{\text {an }}{ }^{\prime}}{A_{0}}$} \\
\hline IN & OUT & & & & & & & & & & & \\
\hline $8-18-75$ & $8-19-75$ & 1 & 1 & 30 & $0.9 n 7$ & $3.04+2$ & $<2$ & & $7.55-8$ & & & \\
\hline $8-19-75$ & $8-20-75$ & 1 & 2 & 29 & 0.911 & $4.7+1$ & & & $1.16-8$ & & & \\
\hline $8-20-75$ & $8-21-75$ & 1 & 3 & $2 B$ & .0 .914 & $3.6+1$ & & & $8.87-9$ & & & \\
\hline $8-21-75$ & $8-22-75$ & 1 & 4 & 27 & 0.917 & $2.0+1$ & & & $4.91-9$ & & & \\
\hline $8-22-75$ & $8-25-75$ & 3 & 7 & 24 & 0.926 & $3.3+1$ & & . & $8.03-9$ & & & \\
\hline $8-25-75$ & $9-02-75$ & 8 & 15 & 31 & 0.905 & $4.6+1$ & & & $1.74-8$ & & & \\
\hline $9-02-75$ & $9-08-75$ & 6 & 21 & 25 & 0.923 & $1.8+1$ & & & $4.39-?$ & & & \\
\hline $9-08-75$ & $9-18-75$ & 10 & 37 & 26 & $0.92 n$ & $2.5+1$ & & & $6.12-9$ & & & \\
\hline $9-18-75$ & $10-17-75$ & 29 & 60 & 19 & 0.942 & $9.8 \quad 0$ & & & $2.34-9$ & & & \\
\hline $10-17-75$ & $11-18-75$ & 32 & 92 & 196 & 0.449 & 0.80 & & & $4.01-10$ & & & \\
\hline $11-18-75$ & $12-17-75$ & 29 & 121 & 37 & 0.886 & $3.86 \quad 0$ & & & $0.81-10$ & & & \\
\hline $12-17-75$ & $1-15-76$ & 29 & 150 & 8 & 0.975 & $\begin{array}{rr}6.69 & 0 \\
\end{array}$ & & & $1.43-\mathrm{a}$ & & & \\
\hline & & & & & & & & & & & & \\
\hline & & & & & & & & & & & & \\
\hline & & & & & & & & & & & & \\
\hline & & 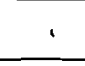 & & & & & & & & & & \\
\hline & & & & & & & & & & & & \\
\hline
\end{tabular}

- Concentration in leachate, show units.

$\cdots a_{1}=$ corrected concentration $\times V_{L} \times$ factor to convert to same units as $A_{0}$. 
$\because$

LEACH TEST IDENTIFICATION NUMBER

Z-3

LABORATORY WHERE TESTS PERFORMED

ANALYST

DATE RESULTS REPORTS

Part C. Experimental Data

Constituent Analyzed, A

Initial Amount in Sample $A_{0}$

Curium 244

$0.027985 \mathrm{Ci}$

\begin{tabular}{|c|c|c|c|c|c|c|c|c|c|c|c|c|}
\hline \multicolumn{2}{|c|}{ TIME AND DATE } & \multirow{2}{*}{$I_{n}$} & \multirow{2}{*}{$\underset{T}{\operatorname{rotal}} \cdot$} & \multirow{2}{*}{$\begin{array}{l}\text { DAYS } \\
\text { DECAY }\end{array}$} & \multirow{2}{*}{$\begin{array}{l}\text { DECAY } \\
\text { FACTOR }\end{array}$} & \multirow{2}{*}{$\begin{array}{c}\text { AS } \\
\text { ANALYZED } \\
1 \mathrm{~d} / \mathrm{m} / \mathrm{ml})^{\cdots} \\
\end{array}$} & \multirow{2}{*}{$\begin{array}{c}\text { BLANK } \\
\mathrm{d} / \mathrm{m} / \mathrm{ml}\end{array}$} & \multirow{2}{*}{$\begin{array}{l}\text { CORAECTED } \\
\text { CONC. } \\
1\end{array}$} & \multirow{2}{*}{$a_{1} \cdots$} & \multirow{2}{*}{$\frac{a_{1}}{A_{0}}\left(\frac{w}{S I_{n}}\right)$} & \multirow{2}{*}{$\sum a_{1}$} & \multirow{2}{*}{$\frac{\operatorname{man}^{\prime}}{A_{0}}$} \\
\hline IN & our & & & & & & & & & & & \\
\hline $8-18-75$ & $8-19-75$ & 1 & 1 & & & $3.18+2$ & $<2$ & & $7.16-8$ & $1.14-6$ & $7.16-8$ & $2.56-6$ \\
\hline $8-19-75$ & $8-20-75$ & 1 & 2 & & & $4.0+1$ & & & $9.01-9$ & $1.43-7$ & $8.06-8$ & $2.88-6$ \\
\hline $8-20-75$ & $8-21-75$ & 1 & 3 & & . & $4.5+1$ & & & $1.01 \cdot-8$ & $1.61-7$ & $9.08-8$ & $3.24-6$ \\
\hline $8-21-75$ & $8-22-75$ & 1 & 4 & & & $2.0+1$ & & & $4.50-9$ & $7.4-8$ & $9.53-8$ & $3.40-6$ \\
\hline $8-22-75$ & $8-25-75$ & 3 & 7 & & & $2.4+1$ & & $\cdots$ & $5.41-9$ & $2.86-8$ & $1.01-7$ & $3.60-6$ \\
\hline $8-25-75$ & $9-02-75$ & 8 & 15 & & & $6.0+1$ & & & $1.35-8$ & $2.68-8$ & $1.14-7$ & $4.08-6$ \\
\hline $9-02-75$ & $9-08-75$ & 6 & 2] & & & $1.8+1$ & & & $4.05-9$ & $1.07-8$ & $1.18-7$ & $4.22-6$ \\
\hline $9-08-75$ & $9-18-75$ & 10 & 31 & & & $3.7+1$ & & & $8.33-9$ & $1.32-8$ & $1.27-7$ & $4.52-6$ \\
\hline $9-18-75$ & $10-17-75$ & 29 & 60 & & & $1.5+1$ & & & $3.38-9$ & $1.85-9$ & $1.30-7$ & $4.64-6$ \\
\hline $10-17-75$ & $11-18-75$ & 32 & 92 & & & 2.2 & & & $4: 96-10$ & $2.45-10$ & $1.30-7$ & $4.66-6$ \\
\hline $11-18-75$ & $12-17-75$ & 29 & 121. & & & 9.650 & & & $2.17-9$ & $1.19-9$ & $1.33-7$ & $4.74-6$ \\
\hline $12-17-75$ & $1-15-76$ & 29 & 150 & & & $1.50+1$ & & & $3.38-9$ & $1.85-9$ & $1.36-7$ & $4.86-6$ \\
\hline & & & & & & & & & & & & \\
\hline & & & & & & & & & & & & \\
\hline & & & & & & & & & i & & & \\
\hline & & $\cdot$ & & & & & & & & & & \\
\hline & & & & & & & & & & & & \\
\hline
\end{tabular}




\section{LEACH TEST RESULTS}

LEACH TEST IDENTIFICATION NUMBER $\frac{Z-4}{\text { LABORATORY WHERE TESTS PERFORMED Battelle-Northwest }}$
LANALYST

ANALYST

DATE RESULTS REPORTED

\section{Part A. Description of Leach Specimen}

Specimen Identification Number $7-n$

Proportion of Waste Incorporated in Mixture Irradiated LWR fuel pellet_Weight \%

or___ Volume\%

(Based on initial volumes)

Type of W3ste, Chemical and Radioisotopic Composition, and Specific Activity of the Waste

Type and Composition of the Immobilization Material

Preparaiion of Specimen.

Storage Conditions

Appearance: 
LEACH TEST RESULTS

LEACH TEST IDENTIFICATION NUMBER $\frac{7-4}{\text { LABORATORY WHERE TESTS PERFORMED B Battelle-Northwest }}$
LAB lo

ANALYST

DATE RESULTS REPORTED

Part B. Description of Leach Test Procedure

Sample Preparation*

Initial Weight of Sample, $W \quad 4.3392 \mathrm{am}$

Volume of Sample, $V$

Surface Area of Sample, $\mathrm{s} \quad 9.82 \mathrm{~cm}^{\text {? }}$

Method of Surface Area Determination aeometric approximation

Diagram of Leach Apparatus: Paiqe type

Leachant 324 Building water

Leachant Volume, $v_{1} \quad 500 \mathrm{ml}$

Sampling Procedure drain Teachant and refill at samplina

Analytical Techniques:

$\mathrm{pH}$ Instrument Identification and Calibration

Constituent $a_{1}$, Analytical Procedure, Standard Deviation of Method

Constituent $a_{2}$. Analytical Procedure, Standard Deviation of Method

*If different from "Preparation of Specimen" in Part A. 
LEACH TEST IDENTIFICATION NUMBER

'LABORATORY WHERE TESTS PERFORMED

ANALYST

DATE RESULTS REPORTS

Part C. Experimental Data

Constituent Analyzed, A

Cesium- 137

Initial Amount in Sample $A_{0} \quad 0.7297 \mathrm{Ci}$

\begin{tabular}{|c|c|c|c|c|c|c|c|c|c|c|c|c|c|c|c|c|}
\hline \multicolumn{2}{|c|}{ TIME AND DATE } & \multirow{2}{*}{$t_{n}$} & \multirow{2}{*}{$\underset{T}{\text { TOTAL }}$} & \multirow{2}{*}{$\begin{array}{l}\text { DAYS } \\
\text { DECAY }\end{array}$} & \multirow{2}{*}{$\begin{array}{l}\text { DECAY } \\
\text { FACTOR }\end{array}$} & \multirow{2}{*}{$\begin{array}{c}\text { AS } \\
\text { ANALYZED } \\
\text { (t/m/ml } / \cdots\end{array}$} & \multirow{2}{*}{$\begin{array}{c}\text { BLANK } \\
(\mathrm{d} / \mathrm{m} / \mathrm{ml})^{\prime}\end{array}$} & \multirow{2}{*}{$\begin{array}{c}\text { CORAECTED } \\
\text { CONC. } \\
1\end{array}$} & \multirow{2}{*}{\multicolumn{2}{|c|}{$a_{1} \cdots$}} & \multirow{2}{*}{\multicolumn{2}{|c|}{$\frac{a_{1}}{A_{0}}\left(\frac{w}{S t_{n}}\right)$}} & \multirow{2}{*}{\multicolumn{2}{|c|}{$\sum \mathbf{a}_{1}$}} & \multirow{2}{*}{\multicolumn{2}{|c|}{$\frac{\sum_{\text {an }}{ }^{\prime}}{A_{0}}$}} \\
\hline $\mathbb{N}$ & OUT & & & & & & & & & & & & & & & \\
\hline $8-18-75$ & $8-19-75$ & 1 & 1 & & & $2.50+7$ & $9 . .7 n+?$ & & 5.63 & -3 & 3.40 & -3 & 5.63 & -3 & 7.72 & -3 \\
\hline $8-19-75$ & $8-20-75$ & 1 & 2 & & & $5.51+6$ & & & 1.24 & -3 & $7.4^{n}$ & -4 & 6.97 & -3 & 9.41 & -3 \\
\hline $8-20-75$ & $8-21-75$ & 1 & 3 & & & $2.97+6$ & & & 6.69 & -4 & 4.54 & -4 & 7.54 & -3 & 1.73 & -2 \\
\hline $8-21-75$ & $8-22-75$ & 1. & 4 & & & $2.32+6$ & & & 5.23 & -4 & 3.16 & -4 & $8 . n 6$ & -3 & $1.1 n$ & -2 \\
\hline $8-22-75$ & $8-25-75$ & 3 & 7 & & & $3.68+6$ & & $\therefore$ & 8.29 & -4 & 1.67 & -4 & 8.90 & -3 & 1.22 & -2 \\
\hline $8-25-75$ & $9-2-75$ & 8 & 15 & & & $7.35+6$ & & & 1.66 & -3 & 1.24 & -4 & 1.06 & -2 & 1.45 & -2 \\
\hline $9-2-75$ & $9-8-75$ & 6 & 21 & & & $3.8^{0}+6$ & & & 3.76 & -4 & 3.92 & -5 & 1.14 & -2 & 1.57 & -2 \\
\hline $9-8-75$ & $9-18-75$ & 10 & 31 & & & $3.52+6$ & & . & 7.93 & -4 & 1.79 & -5 & 1.22 & -2 & 1.67 & -2 \\
\hline $9-18-75$ & $10-17-75$ & 29 & 60 & & & $4.31+6$ & & & 0.71 & -1 & 2.72 & -5 & 1.32 & $-?$ & 1.81 & $-?$ \\
\hline $10-17-75$ & $11-18-75$ & 32 & 92 & & & $? . n 7+6$ & & & 4.66 & -1 & $9.3 n$ & -6 & 1.37 & -2 & 1.87 & -2 \\
\hline $11-18-75$ & $12-17-75$ & 29 & 121 & & & $1 . n n+f$ & & & $? .46$ & -4 & 5.11 & $-f$ & $1.3^{n}$ & -2 & 1.01 & -2 \\
\hline $12-17-75$ & $1-15-76$ & 29 & 150 & & & $1.07+f$ & & & $? .41$ & -1 & 5.03 & -6 & 1.41 & -2 & 1.34 & -2 \\
\hline-- & & & & & & & & & & & & & & & & \\
\hline & & & & & & & & & & & & & & & & \\
\hline & & & & & & & & & & & & & & & & \\
\hline & & & & & & & & & & & & & & & & \\
\hline & & & & & & & & & & & & & & & & \\
\hline & & & & & & & & & & & & & & & & \\
\hline
\end{tabular}

- Concentration in leachate, show units.

$\cdots \Delta_{1}=$ corrected concentration $\times V_{L}$ x factor to conven lo same units as $A_{0}$. 


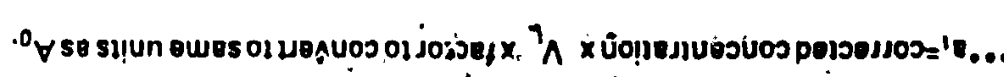

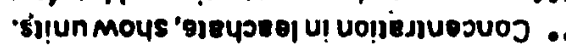

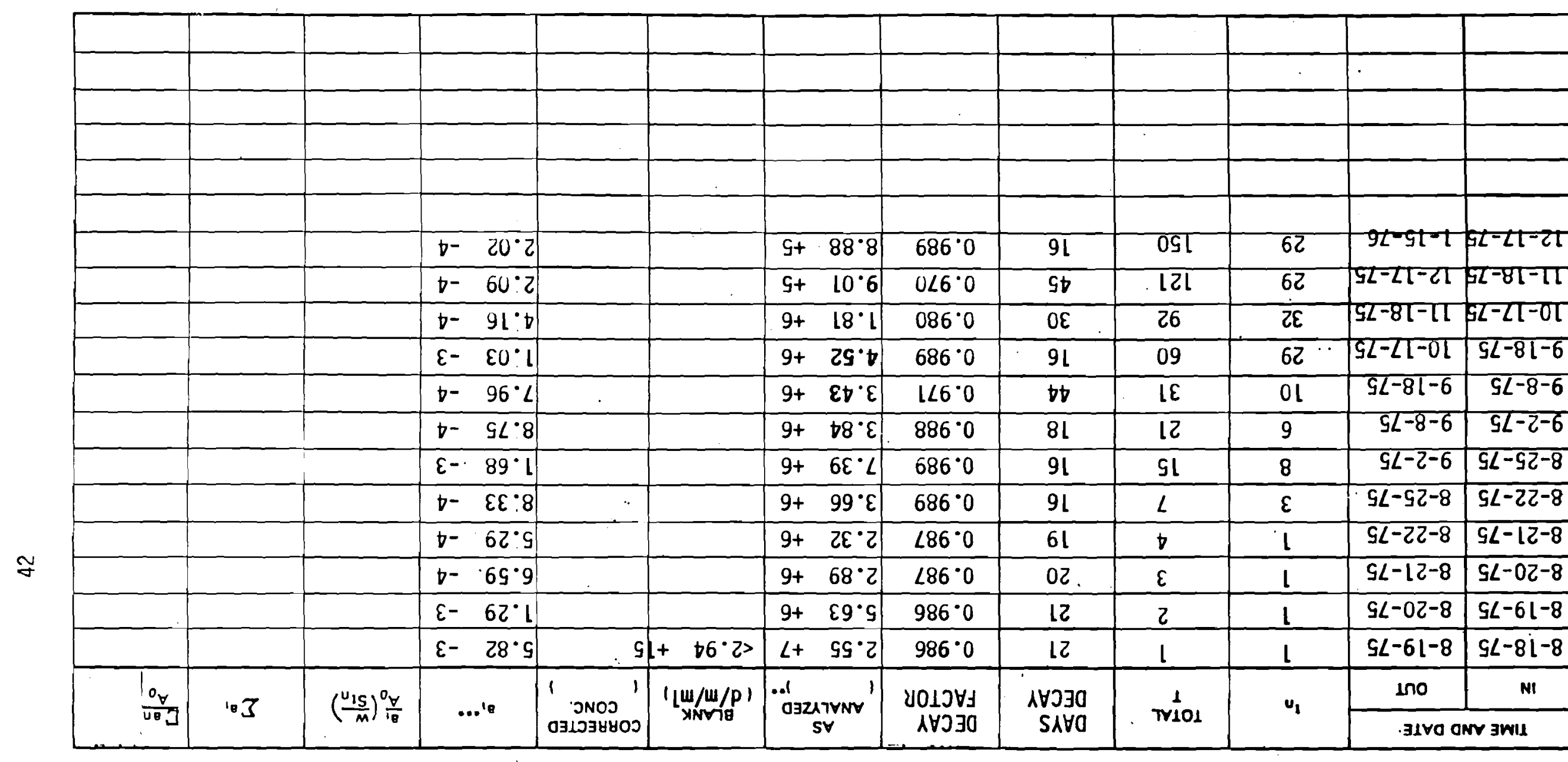

$12+80^{\circ}$ tE (-un! sa)
${ }^{\circ} \forall$ e|dures U! junow $\forall$ Ie!!!U

$\forall$ 'pezlleu $\forall$ iven!ysuos

mog lozuouịjodx

SLHOd3Y SL7กS3Y J1V

1SATVN

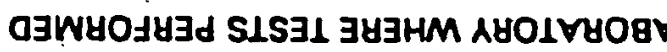




\section{LEACH TEST RESỤLTS}

$\because$

LEACH TEST IDENTIFICATION NUMBER

Z-4

LABORATORY WHERE TESTS PERFORMED

ANALYST

DATE RESULTS REPORTS

Part C. Experimental Data

Constituent Analyzed, A Plutonium-23a+?4n

Initial Amount in Sample $A_{0}$

$3.6566 \times 1 n^{-3} \mathrm{Ci}$

\begin{tabular}{|c|c|c|c|c|c|c|c|c|c|c|c|c|c|c|c|c|}
\hline \multicolumn{2}{|c|}{ TIME AND DATE } & \multirow{2}{*}{$\ln ^{n}$} & \multirow{2}{*}{$\underset{T}{\text { TOTAL }}$} & \multirow{2}{*}{$\begin{array}{l}\text { DAYS } \\
\text { DECAY }\end{array}$} & \multirow{2}{*}{$\begin{array}{l}\text { DECAY } \\
\text { FACTOR }\end{array}$} & \multirow{2}{*}{$\begin{array}{c}\text { AS } \\
\text { ANALYZED } \\
1 \mathrm{~d} / \mathrm{m} / \mathrm{m})^{\cdots}\end{array}$} & \multirow{3}{*}{$\frac{\begin{array}{c}\text { BLANK } \\
\mathrm{d} / \mathrm{m} / \mathrm{ml})^{\prime}\end{array}}{<?}$} & \multirow{2}{*}{$\begin{array}{l}\text { CORRECTED } \\
\text { CONC. } \\
1\end{array}$} & \multirow{2}{*}{\multicolumn{2}{|c|}{$a_{1} \cdots$}} & \multirow{2}{*}{\multicolumn{2}{|c|}{$\frac{a_{1}}{A_{0}}\left(\frac{w}{S t_{n}}\right)$}} & \multirow{2}{*}{\multicolumn{2}{|c|}{$\sum \mathrm{a}_{1}$}} & \multirow{2}{*}{\multicolumn{2}{|c|}{$\frac{\sum_{\text {an }} !}{A_{0}}$}} \\
\hline IN & our & & & & & & & & & & & & & & & \\
\hline $8-18-75$ & $8-19-75$ & 1 & 1 & & & $5 . n 2+2$ & & & 1.13 & $\begin{array}{l}-7 \\
\end{array}$ & 1.36 & -5 & 1.13 & -7 & $3 . n 9$ & -5 \\
\hline $8-19-75$ & $8-20-75$ & 1 & 2 & & & $5.32+?$ & & & 1.20 & -7 & 1.45 & -5 & 2.33 & -7 & 6.35 & -5 \\
\hline $8-20-75$ & $8-21-75$ & 1 & 3 & & & $4.30+?$ & & & $? .0$ & -8 & 1.10 & -5 & 3.32 & -7 & 9.75 & -5 \\
\hline $8-21-75$ & $8-22-75$ & 1 & 4 & & & $3.93+?$ & & & 3.95 & -8 & $1 . n 7$ & -5 & $4 . ? 0$ & & 1.15 & -4 \\
\hline $8-22-75$ & $8-25-75$ & 3 & 7 & & & $4.91+2$ & & . & 1.11 & -7 & 4.44 & -6 & 5.31 & -7 & 1.45 & -4 \\
\hline $8-25-75$ & $9-2-75$ & 8 & 15 & & & $1.56+3$ & & & 3.51 & -7 & $5.3 n$ & .6 & 8.82 & -7 & 2.42 & -4 \\
\hline $9-2-75$ & $9-8-75$ & 6 & 21 & & & $7.30+?$ & & & 1.66 & -7 & 3.36 & -6 & 1.05 & -6 & 2.87 & -4 \\
\hline $9-8-75$ & $9-18-75$ & 10 & 31 & & & $5.6 .3+?$ & & $\cdot$ & 1.27 & -7 & 1.53 & -6 & 1.18 & -6 & $3.2 ?$ & -4 \\
\hline $9-18-75$ & $10-17-75$ & 29 & 60 & & & $5.1+1$ & & & 1.15 & -8 & 4.78 & -8 & 1.19 & -6 & 3.24 & -4 \\
\hline $10-17-75$ & $11-18-75$ & 32 & 92 & & & $2.51+2$ & & & 5.65 & -8 & 2.13 & -7 & 1.25 & -6 & 3.41 & -4 \\
\hline $11-18-75$ & $12-17-75$ & 29 & 121 & & & $2.6 A+?$ & & & 5.05 & $-?$ & 2.48 & -7 & 1.31 & -6 & 3.57 & -4 \\
\hline $12=17-75$ & $1-15-76$ & 29 & 150 & & & $3.27+2$ & & & 7.36 & -8 & 3.05 & -7 & 1.39 & $-F$ & 3.77 & -4 \\
\hline$r$ & & & & & & & & & & & & & & & & \\
\hline & & & & & & & & & & & & & & & & \\
\hline & & & & & & & & & & & & & & & & \\
\hline & & & & & & & & & & & & & & & & \\
\hline & & & & & & & & & & & & & & & & \\
\hline & & & & & & & & · & & & & & & & & \\
\hline
\end{tabular}

- $a_{a_{1}}$-corracted concentration $\times V_{L} \times$ factor to convert to same units as $A_{O}$. 
$\because$

LEACH TEST IDENTIFICATION NUMBER

Z-4

LABORATORY WHERE TESTS PERFORMED

ANALYST

DATE RESULTS REPORTS

Part C. Experimental Data

Constituent Analyzed, A
Initial Amount in Sample $A_{0} \quad 1.5276 \times 10^{-2} \mathrm{Ci}$

$\mathrm{Ci}$

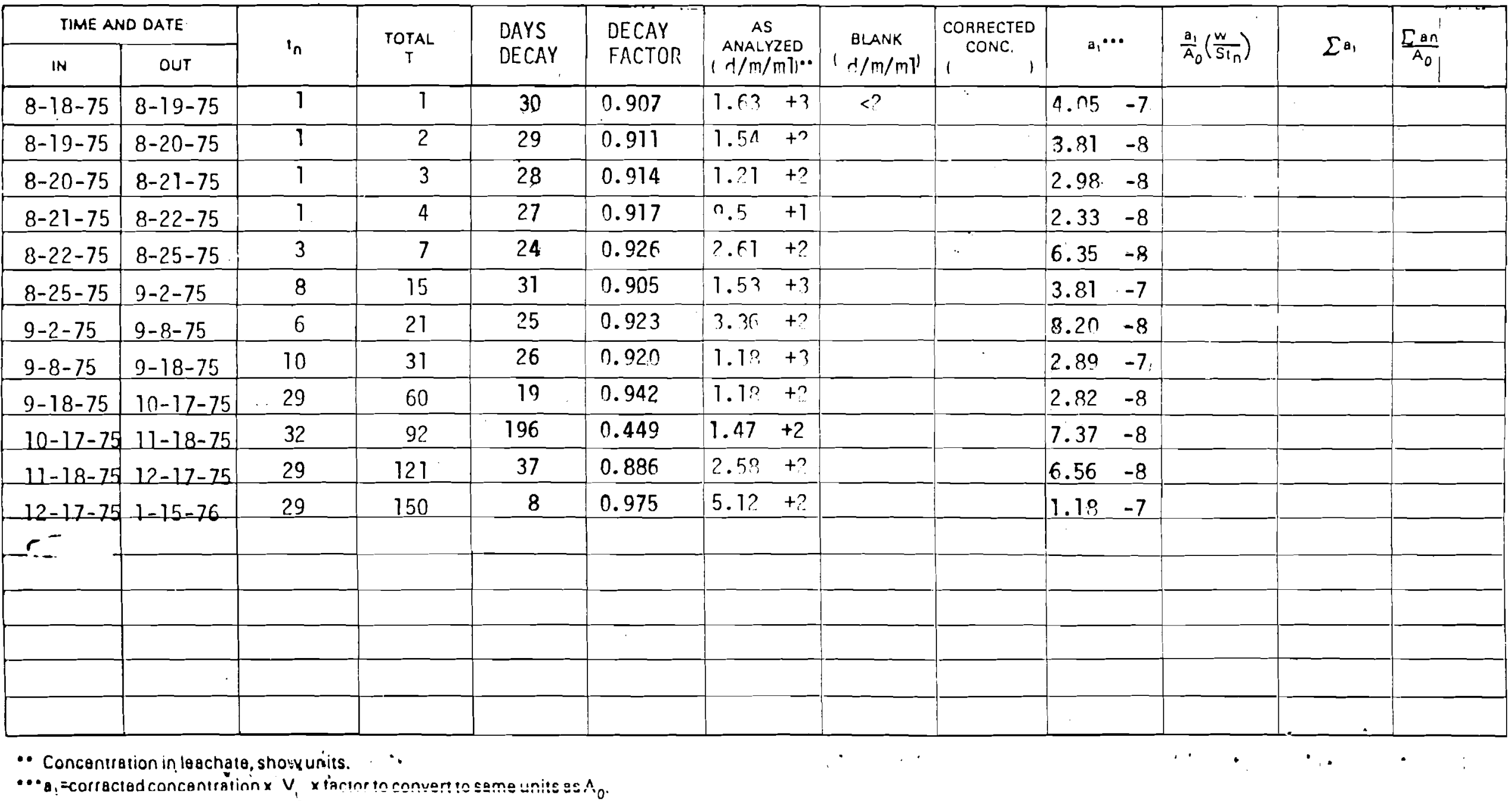


ANALYST

DATE RESULTS REPORTS

\section{Part C. Experimental Data}

Constituent Analyzed, A $\quad$ Curium-244

Initial Amount in Sample $A_{0} \quad 0.024054 \mathrm{Ci}$

\begin{tabular}{|c|c|c|c|c|c|c|c|c|c|c|c|c|c|c|c|c|}
\hline \multicolumn{2}{|c|}{ TIME AND DATE } & \multirow{2}{*}{$t_{n}$} & \multirow{2}{*}{$\underset{T}{\text { TOTAL }}$} & \multirow{2}{*}{$\begin{array}{l}\text { DAYS } \\
\text { DECAY }\end{array}$} & \multirow{2}{*}{$\begin{array}{l}\text { DECAY } \\
\text { FACTOR }\end{array}$} & \multirow{2}{*}{$\begin{array}{c}\text { AS } \\
\text { ANALYZED } \\
(\mathrm{d} / \mathrm{m} / \mathrm{ml})^{*}\end{array}$} & \multirow{2}{*}{$\begin{array}{c}\text { BLANK } \\
(\mathrm{d} / \mathrm{m} / \mathrm{m} l)\end{array}$} & \multirow{2}{*}{$\begin{array}{l}\text { CORRECTED } \\
\text { CONC. } \\
1\end{array}$} & \multirow{2}{*}{\multicolumn{2}{|c|}{$a_{1} \cdots$}} & \multirow{2}{*}{\multicolumn{2}{|c|}{$\frac{a_{1}}{A_{0}}\left(\frac{w}{S t_{n}}\right)$}} & \multirow{2}{*}{\multicolumn{2}{|c|}{$\sum a_{1}$}} & \multirow{2}{*}{\multicolumn{2}{|c|}{$\frac{\sum_{\text {an }}^{\prime}}{A_{0}}$}} \\
\hline IN & OUT & & & & & & & & & & & & & & & \\
\hline $8-18-75$ & $8-19-75$ & 1 & 1 & & & $1.67+3$ & $<?$ & & 3.76 & -7 & 6.01 & -6 & 3.76 & -7 & 1.56 & -5 \\
\hline $8-19-75$ & $8-20-75$ & 1 & 2 & & & $1.17+?$ & $<2$ & & 2.54 & -8 & 4.34 & -7 & 4.02 & -7 & 1.677 & -5 \\
\hline $8-20-75$ & $8-21-75$ & 1 & 3 & & & $1.13+?$ & $<?$ & & 2.54 & -9 & 7.69 & -7 & 4.28 & -7 & 1.78 & -5 \\
\hline $8-21-75$ & $8-22-75$ & 1. & 4 & & & $0.7+1$ & $<2$ & & 2.13 & $-?$ & $4 . n ?$ & -7 & 4.50 & -7 & 1.87 & -5 \\
\hline $8-22-75$ & $8-25-75$ & 3 & 7 & & & $2.5 n+?$ & $<2$ & 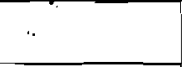 & 5.63 & -8 & 3.45 & -7 & 5.06 & -7 & 2.17 & -5 \\
\hline $8-25-75$ & $9-2-75$ & 8 & 15 & & & $1.50+3$ & $<2$ & & 3.59 & -7 & 8.23 & -7 & 8.64 & -7 & 3.50 & -5 \\
\hline $9-2-75$ & $9-8-75$ & 6 & 21 & & & $3.38+?$ & $<?$ & & 7.61 & -8 & 2.33 & -7 & $9.1 n$ & -7 & 3.01 & -5 \\
\hline $9-8-75$ & $9-18-75$ & 10 & 31 & & & $1.17+?$ & $<?$ & & 2.54 & -7 & 4.24 & -7 & 1.21 & $-E$ & $5.0 n$ & -5 \\
\hline $9-18-75$ & $10-17-75$ & 29 & 60 & & & $1.14+?$ & $<2$ & & 3.24 & -8 & $? .06$ & -9 & 1.24 & -6 & 5.14 & -5 \\
\hline $10-17-75$ & $11-18-75$ & 32 & 92 & & & $3.82+2$ & & & 8.60 & -8 & 4.94 & -8 & 1.33 & -6 & 5.51 & -5 \\
\hline $11-18-25$ & $12-17-75$ & 29 & $121^{\circ}$ & & & $1.53+0$ & $<?$ & & $1.0 ?$ & -7 & 6.47 & -8 & 1.43 & $-h$ & 5.94 & -5 \\
\hline $12-17-75$ & $1-15-76$ & 29 & 150 & & & $2.73+2$ & $<2$ & & 1.07 & -7 & 1.25 & -7 & 1.68 & -6 & 6.76 & -5 \\
\hline$r$ & & & & & & & & & & & & & & & & \\
\hline & & & & & & & & & & & & & & & & \\
\hline & & & & & & & & & & & & & & & & \\
\hline & & & & & & & & & & & & & & & & \\
\hline & & & & & & & & & & & & & & & & \\
\hline & & & & & & & & & & & & & & & & \\
\hline
\end{tabular}

-. Concentration in leachate, show units.

$\cdots a_{1}=$ corractad concentration $\times V$ x factor to convert to sama unitc as $A$ 
$\because$

LEACH TEST IDENTIFICATION NUMBER

$Z-4$

$-$

LABORATORY WHERE TESTS PERFORMED

ANALYST

DATE RESULTS REPORTS

Part C. Experimental Data

Constituent Analyzed, A

Antimony-125

Initial Amount in Sample $A_{0}$

$3.084 \times 10^{-2} \mathrm{Ci}$

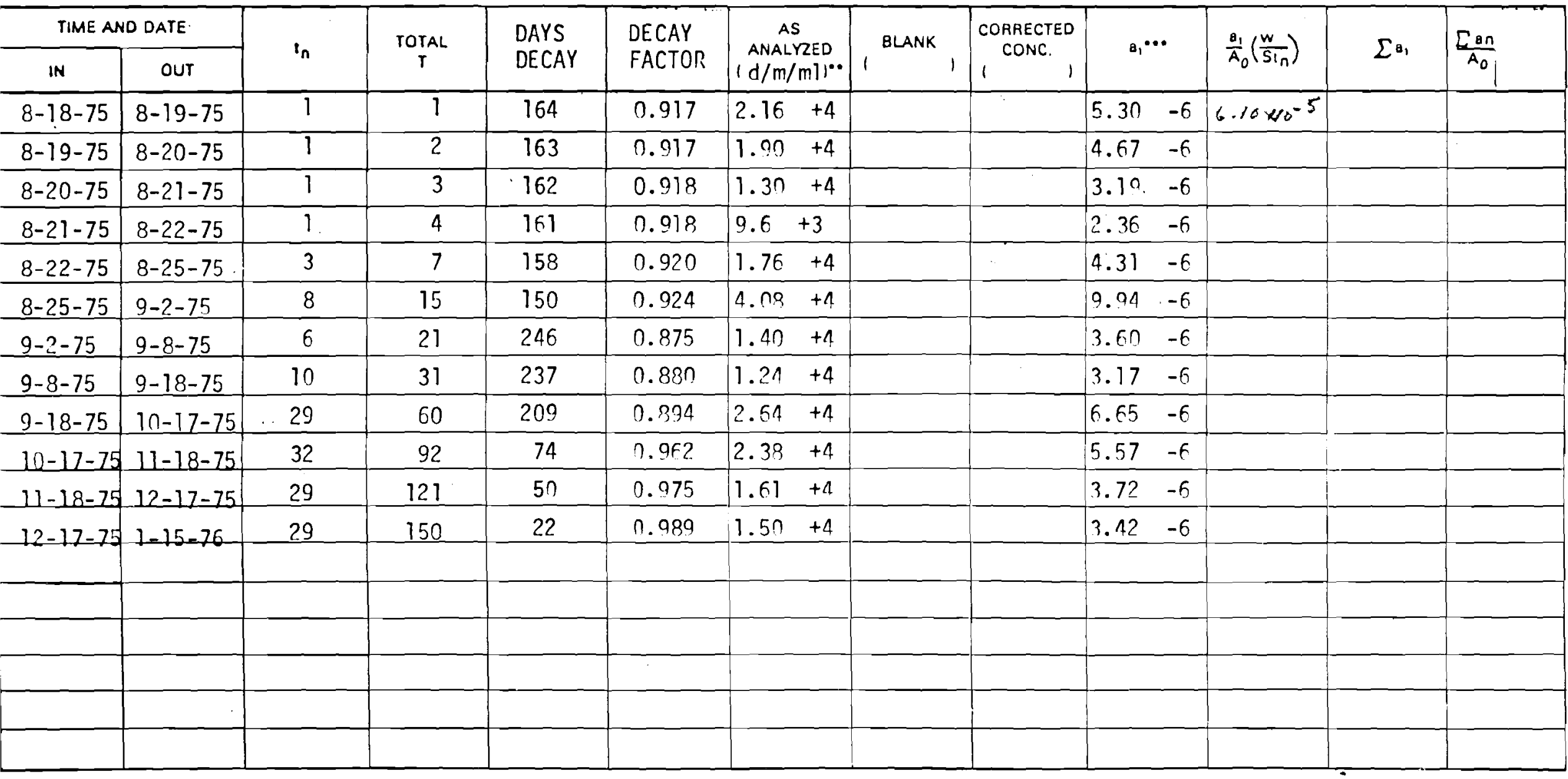

- Concentration in leachate, show urits.

$\cdots_{a_{1}}=$ correcied conzentratieri $\times . V_{L}$ x factor to convert to same units as $A_{n}$ 
$\because$

EACH TEST IDENTIFICATION NUMBER

ABORATORY WHERE TESTS PERFORMED

NALYST

ATE RESULTS REPORTS

an C. Experimental Date

Constituent Analyzed, A $\quad$ Europi um-154

Initial Amount in Sample $A_{0}-0.052714 \mathrm{Ci}$

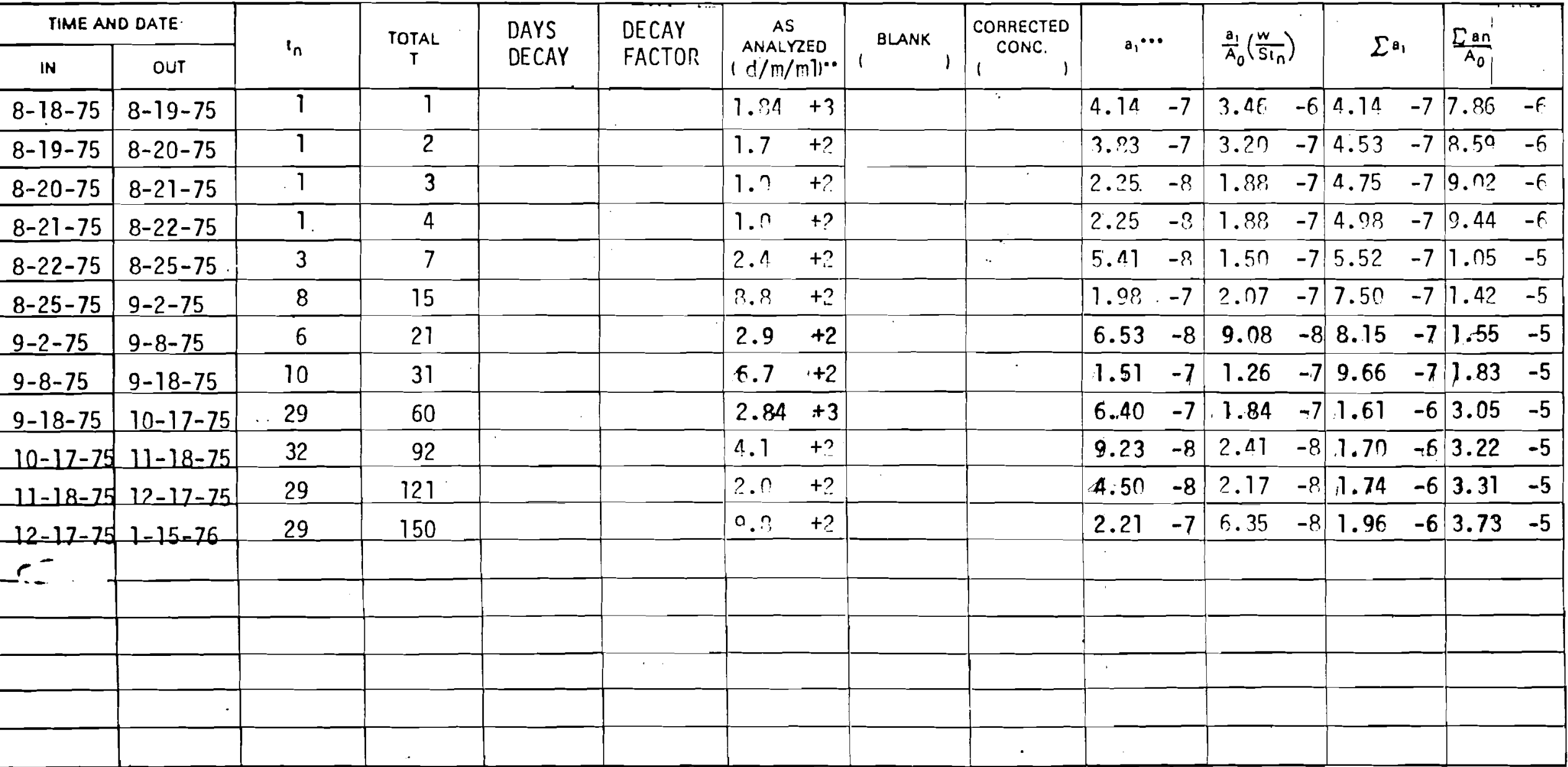

- Concenustion in leachate, show units.

*.. $a_{1}=$ correcied concentration $\times V_{b} \times$ factor to convert co same units as $A_{0}$. 
$\because$

LEACH TEST IDENTIFICATION NUMBER

Z-4

LABORATORY WHERE TESTS PERFORMED

ANALYST

DATE RESULTS REPORTS

Part C. Experimental Data

Constituent Analyzed, A Ruthen ium-106

Initial Amount in Sample $A_{0} \quad 7.509 \times 10^{-1} \mathrm{Ci}$

\begin{tabular}{|c|c|c|c|c|c|c|c|c|c|c|c|c|c|}
\hline \multicolumn{2}{|c|}{ TIME AND DATE } & \multirow{2}{*}{ 'n } & \multirow{2}{*}{$\underset{T}{\text { TOTAL }}$} & \multirow{2}{*}{$\begin{array}{l}\text { DAYS } \\
\text { DECAY }\end{array}$} & \multirow{2}{*}{$\begin{array}{l}\text { DECAY } \\
\text { FACTOR }\end{array}$} & \multirow{2}{*}{$\begin{array}{c}\text { AS } \\
\text { ANALYZED } \\
(\mathrm{d} / \mathrm{m} / \mathrm{m}))^{\circ}\end{array}$} & \multirow{2}{*}{$1^{\text {BLANK }}$} & \multirow{2}{*}{$\begin{array}{l}\text { CORRECTED } \\
\text { CONC. } \\
1\end{array}$} & \multirow{2}{*}{\multicolumn{2}{|c|}{$a_{1} \cdots$}} & \multirow{2}{*}{$\frac{B_{1}}{A_{0}}\left(\frac{w}{S_{I_{n}}}\right)$} & \multirow{2}{*}{$\sum a_{1}$} & \multirow{2}{*}{$\frac{\sum_{\text {on }}}{A_{0}}$} \\
\hline IN & OUT & & & & & & & & & & & & \\
\hline $8-18-75$ & $8-19-75$ & 1 & 1 & 164 & 0.917 & $5.39+4$ & & & 1.32 & -5 & & & \\
\hline $8-19-75$ & $8-20-75$ & 1 & 2 & 163 & 0.917 & $1.98+4$ & & & 4.86 & & & & \\
\hline $8-20-75$ & $8-21-75$ & 1 & 3 & 162 & 0.918 & $1.4 n+4$ & & & 3.43 & -6 & & & \\
\hline $8-21-75$ & $8-22-75$ & 1 & 4 & 161 & 0.918 & $1.24+4$ & & & $3 . n 4$ & & & & \\
\hline $8-22-75$ & $8-25-75$ & 3 & 7 & 158 & $n .92 n$ & $2 . n+4$ & & . & 4.90 & -6 & & & \\
\hline $8-25-75$ & $9-2-75$ & 8 & 15 & 150 & 0.924 & $3.78+4$ & & & 7.51 & -6 & & & \\
\hline $9-2-75$ & $9-8-75$ & 6 & 21 & 246 & 0.875 & $1.15+4$ & & & 2.96 & -6 & & & \\
\hline $9-8-75$ & $9-18-75$ & 10 & 31 & 237 & 0.880 & $1.10+4$ & & . & 2.81 & & & & \\
\hline $9-18-75$ & $10-17-75$ & 29 & 60 & 209 & 0.894 & $1.94+4$ & & & 4.89 & $-\epsilon$ & & & \\
\hline $10-77-75$ & $11-18-75$ & 32 & 92 & 74 & 0.962 & $1.11+4$ & & & $2.6 n$ & -6 & & & \\
\hline $11-18-75$ & $12-17-75$ & 29 & 121 & 50 & 0.975 & $5.54+3$ & & & 1.28 & -6 & & & \\
\hline $12-17-75$ & $1-15-76$ & 29 & 150 & 22 & 0.989 & $4.1 n+3$ & & & 9.34 & -7 & & & \\
\hline & & & & & & & & & & & & & \\
\hline & & & & & & & & & & & & & \\
\hline & & & & & & & & & & & & & \\
\hline & & & & & & & & & & & & & \\
\hline & & & & & & & & & & & & & \\
\hline
\end{tabular}

- Concentration in leachate, show yhits.

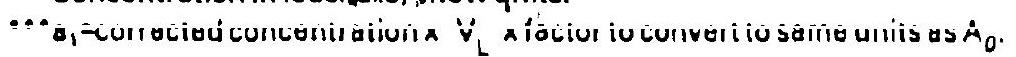


$\because$

LEACH TEST IDENTIFICATION NUMBER

Z-4

LABORATORY WHERE TESTS PERFORMED

ANALYST

DATE RESULTS REPORTS

Part C. Experimental Data

Constituent Analyzed, A Cerium-144

Initial Amount in Sample $A_{0}-1.098 \mathrm{Ci}$

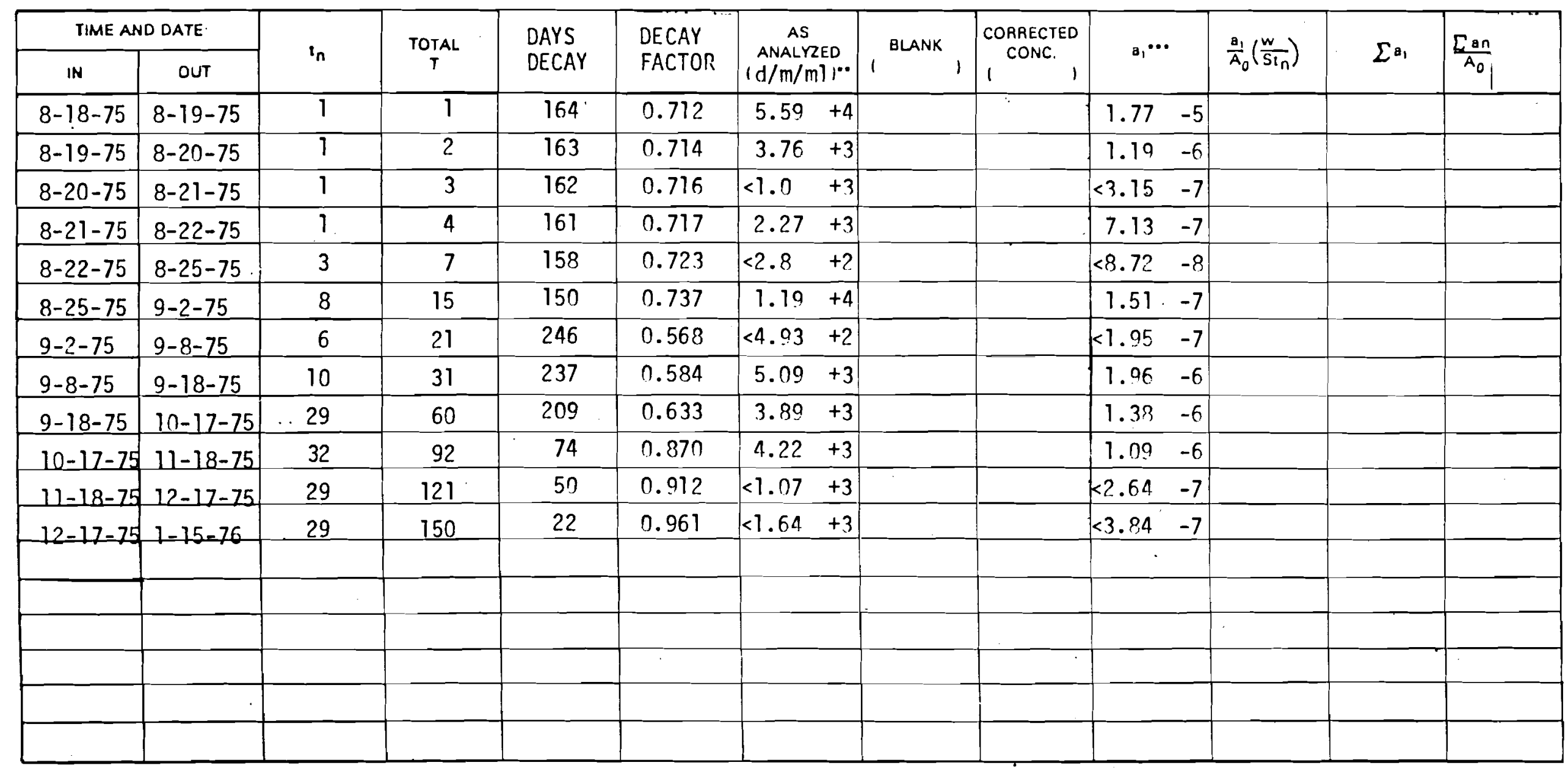

- Concentration in leachate, show units.

$\cdots a_{1}=$ corrected concentration $\times V_{i} \times$ factor to convert to same units as $A_{n}$. 


\section{LEACH TEST RESULTS}

LEACH TEST IDENTIFICATION NUMBER Z Z Z LABORATORY WHERE TESTS PERFORMED Battel le-Northwest

ANALYST

DATE RESULTS REPORTED

Part A. Description of Leach Specimen

Specimen Identification Number Z Z Z -5 Proportion of Waste Incorporated in Mixture_Irradiated LWR fuel pe 1let_Weight \% or Volume\% (Based on initial volumes)

Type of Waste, Chemical and Radioisotopic Composition, and Specific Activity of the Waste

Type and Composition of the Immobilization Material

Preparation of Specimen

Storage Conditions

Appearance: 
LEACH TEST IDENTIFICATION NUMBER 7.-5.5

LABORATORY WHERE TESTS PERFORMED Battelle-Northwest

ANALYST

DATE RESULTS REPORTED

Part B. Description of Leach Test Procedure

Sample Preparation*

Initial Weight of Sample, $W \quad 5.39 ! 1 \mathrm{~nm}$

volume of Sample, $\mathrm{V}$

Surface Area of Sample, $\mathrm{s} \quad 72 . ? \mathrm{~cm}^{2}$

Method of Surface Area Determination neometric approximation

Diagram of Leach Apparatus:___._. Paine type

Leachant $\frac{324 \text { Building water }}{\text { Leachant Volume, } \mathrm{V}_{\mathrm{L}} 50 \mathrm{n} \mathrm{mi}}$
Sampling Procedure drain leachant and refill at samnlina

Analytical Techniques:

$\mathrm{pH}$ Instrument Identification and Calibration

Constituent $a_{1}$, Analytical Procedure, Standard Deviation of Method

Constituent $\mathrm{a}_{2}$, Analytical Procedure, Standard Deviation of Method

*If different from "Preparation of Specimen" in Part A. 
$\because$

LEACH TEST IDENTIFICATION NUMBER

Z-5

LABORATORY WHERE TESTS PERFORMED

ANALYST

DATE RESULTS REPORTS

Part C. Experimental Data

Constituent Analyzed, A

Cesium- 137

Initial Amount in Sample $A_{0} \quad 0.3919 \mathrm{Ci}$

\begin{tabular}{|c|c|c|c|c|c|c|c|c|c|c|c|c|c|c|c|c|}
\hline \multicolumn{2}{|c|}{ TIME AND DATE } & \multirow{2}{*}{$i_{n}$} & \multirow{2}{*}{$\underset{T}{\text { TOTAL }}$} & \multirow{2}{*}{$\begin{array}{l}\text { DAYS } \\
\text { DECAY }\end{array}$} & \multirow{2}{*}{$\begin{array}{l}\text { DECAY } \\
\text { FACTOR }\end{array}$} & \multirow{2}{*}{$\begin{array}{c}\text { AS } \\
\text { ANALYZED } \\
(\mathrm{d} / \mathrm{m} / \mathrm{ml}) . .\end{array}$} & \multirow{2}{*}{$\begin{array}{c}\text { BLANK } \\
(\mathrm{d} / \mathrm{m} / \mathrm{m})^{\prime}\end{array}$} & \multirow{2}{*}{$\begin{array}{l}\text { CORRECTED } \\
\text { CONC. } \\
1\end{array}$} & \multirow{2}{*}{\multicolumn{2}{|c|}{$a_{1} \cdots$}} & \multirow{2}{*}{\multicolumn{2}{|c|}{$\frac{a_{1}}{A_{0}}\left(\frac{w}{S t_{n}}\right)$}} & \multirow{2}{*}{\multicolumn{2}{|c|}{$\sum \mathrm{a}_{1}$}} & \multirow{2}{*}{\multicolumn{2}{|c|}{$\frac{\sum_{\text {an }}}{A_{0}}$}} \\
\hline IN & OUT & & & & & & & & & & & & & & & \\
\hline $8-18-75$ & $8-19-75$ & 1 & 1 & & & $4.133+7$ & $1.62+?$ & & 0.03 & -3 & 4.51 & -3 & $9 . n ?$ & -3 & $1 . n ?$ & -2 \\
\hline $8-19-75$ & $8-20-75$ & 1 & 2 & & & $6.72+6$ & & & 1.51 & -3 & 7.53 & -4 & $1 . n f$ & $-?$ & 1.19 & -2 \\
\hline $8-20-75$ & $8-21-75$ & 1 & 3 & & & $3.3 n+6$ & & & 7.43 & & $3.7^{n}$ & & 1.13 & $-?$ & 1.27 & -2 \\
\hline $8-21-75$ & $8-22-75$ & 1. & 4 & & & $2.1 n+6$ & & & 4.93 & -4 & 2.45 & -4 & 1.18 & $-?$ & 1.33 & -2 \\
\hline $8-22-75$ & $8-25-75$ & 3 & 7 & & & $3 . n 6+6$ & & . & $6 . ? n$ & -4 & 1.14 & -4 & 1.25 & -2 & 1.40 & $-?$ \\
\hline $8-25-75$ & $9-2-75$ & 8 & 15 & & & $3 . n 0+6$ & & & $8 . n n$ & -4 & $5.5 ?$ & -5 & 1.34 & $-?$ & $1.5 n$ & $-?$ \\
\hline $9-2-75$ & $9-8-75$ & 6 & 21 & & & $4.3 n+6$ & & & $1.0 ?$ & -3 & $? . ? 6$ & -5 & 1.45 & $-?$ & $1.5 ?$ & -2 \\
\hline $9-8-75$ & $9-18-75$ & 10 & 31 & & & $3.96+6$ & & & 8.60 & -4 & $4.3 ?$ & -5 & $1.5 a$ & -2 & 1.72 & $-?$ \\
\hline $9-18-75$ & $10-17-75$ & 29 & 60 & & & $? .00+6$ & & & $F .51$ & -1 & $1.1 ?$ & -5 & $1.6 n$ & -2 & $1.5, n$ & $-?$ \\
\hline $10-17-75$ & $11-18-75$ & 32 & 92 & & & $1.75+6$ & & & 2.36 & -1 & 3.68 & -6 & $1.6 ?$ & $-?$ & $1 . ? 2$ & $-?$ \\
\hline $11-18-75$ & $12-17-75$ & 29 & 121 & & & $1.27+f$ & & & 2.11 & -1 & n.on & -6 & 1.6 .5 & -2 & 1.95 & -2 \\
\hline $12-17-75$ & $1-15-76$ & 29 & 150 & & & $1.34+6$ & & & $3 . n ?$ & & 5.19 & -6 & 1.68 & -2 & 1.88 & -2 \\
\hline$r^{-}$ & & & & & & & & & & & & & & & & \\
\hline & & & & & & & & & & & & & & & & \\
\hline & & & & & & & & & & & & & & & & \\
\hline & & & & & & & & & & & & & & & & \\
\hline & & & & & & & & & & & & & & & & \\
\hline & & & & & & & & & & & & & & & & \\
\hline
\end{tabular}

- Concentration ir leachaje show uniss.

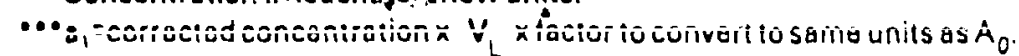




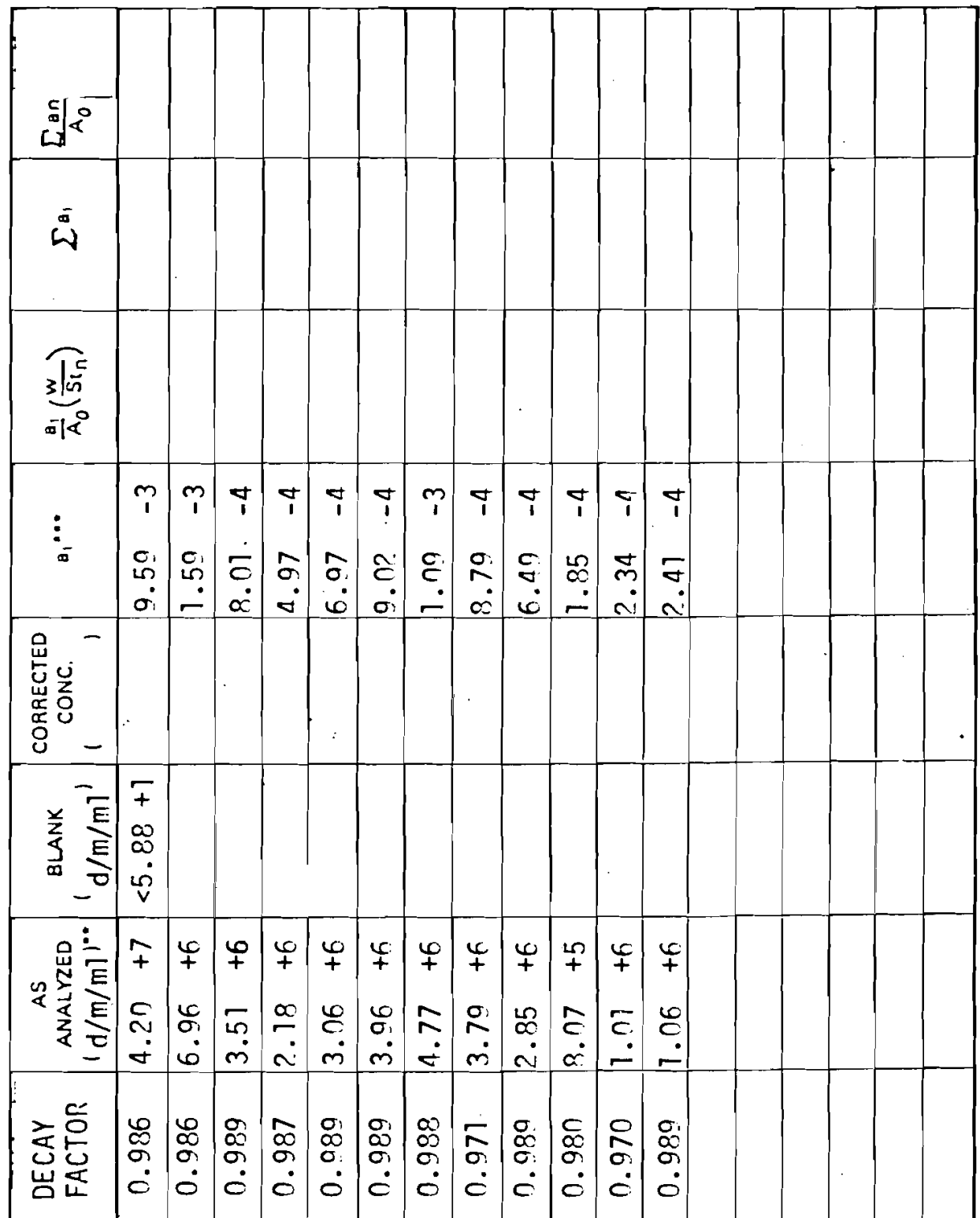

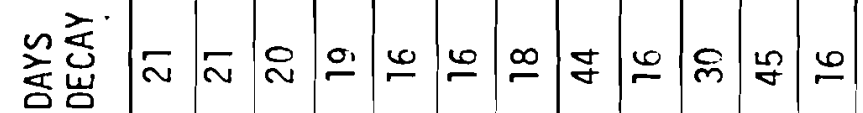

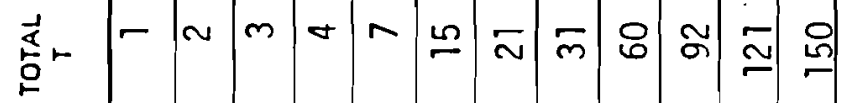

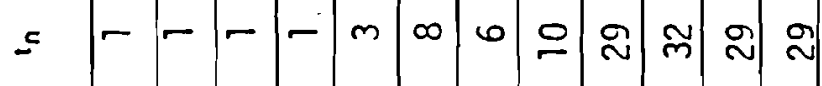

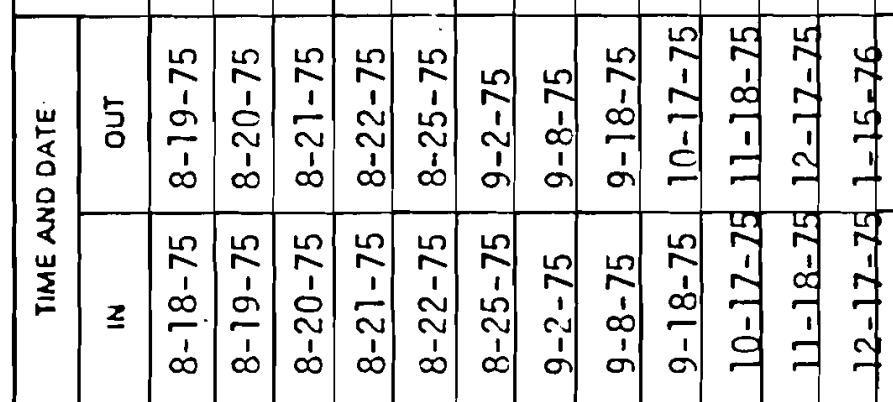


$\because$

LEACH TEST IDENTIFICATION NUMBER

$z-5$

LABORATORY WHERE TESTS PERFORMED

ANALYST

DATE RESULTS REPORTS

Part C. Experimental Data

Constituent Analyzed, A $\quad$ Plutonium-230 + 240

Initial Amount in Sample $A_{0} 4.4698 \times 10^{-3} \mathrm{Ci}$

\begin{tabular}{|c|c|c|c|c|c|c|c|c|c|c|c|c|c|c|c|c|c|}
\hline \multicolumn{2}{|c|}{ TIME AND DATE } & \multirow{2}{*}{$i_{n}$} & \multirow{2}{*}{$\underset{T}{\text { TOTAL }}$} & \multirow{2}{*}{$\begin{array}{l}\text { DAYS } \\
\text { DECAY }\end{array}$} & \multirow{2}{*}{$\begin{array}{l}\text { DECAY } \\
\text { FACTOR }\end{array}$} & \multirow{2}{*}{\multicolumn{2}{|c|}{$\begin{array}{c}\text { AS } \\
\text { ANALYZED } \\
(\mathrm{d} / \mathrm{m} / \mathrm{m})^{* *}\end{array}$}} & \multirow{2}{*}{$\begin{array}{c}\text { BLANK } \\
\left(\mathrm{d} / \mathrm{m} / \mathrm{ml} \mathrm{l}^{\prime}\right.\end{array}$} & \multirow{2}{*}{$\begin{array}{c}\text { CORAECTED } \\
\text { CONC. }\end{array}$} & \multirow{2}{*}{\multicolumn{2}{|c|}{$a_{1} \cdots$}} & \multirow{2}{*}{\multicolumn{2}{|c|}{$\frac{a_{1}}{A_{0}}\left(\frac{w}{S_{i n}}\right)$}} & \multirow{2}{*}{\multicolumn{2}{|c|}{$\sum a_{1}$}} & \multirow{2}{*}{\multicolumn{2}{|c|}{$\frac{\sum \text { an }}{A_{0}}$}} \\
\hline IN & OUT & & & & & & & & & & & & & & & & \\
\hline $8-18-75$ & $8-19-75$ & 1 & 1 & & & 3.25 & $+?$ & $<?$ & & $7.3 ?$ & -8 & 7.24 & -6 & 7.32 & -9 & 1.64 & -5 \\
\hline $8-19-75$ & $8-20-75$ & 1 & 2 & & & 5.8 & +1 & & & 1.31 & $-?$ & 1.29 & -6 & 8.63 & -8 & 1.93 & -5 \\
\hline $8-20-75$ & $8-21-75$ & 1 & 3 & & & 3.02 & $+?$ & & & $9.9,3$ & $-?$ & 8.74 & -6 & 1.74 & -7 & 3.90 & -5 \\
\hline $8-21-75$ & $8-22-75$ & 1 & 4 & & & 3.27 & $+?$ & & & 7.35 & -9 & 7.28 & -6 & $? .48$ & -7 & 5.55 & -5 \\
\hline $8-22-75$ & $8-25-75$ & 3 & 7 & & & 4.83 & $+?$ & & . & 1. no & -7 & 1.08 & -6 & 3.57 & -7 & 8.07 & -5 \\
\hline $8-25-75$ & $9-2-75$ & 8 & 15 & & & 4.0 & $+?$ & & & $1.1^{n}$ & -7 & 1.37 & -6 & 4.67 & -7 & 1.04 & -1 \\
\hline $9-2-75$ & $9-8-75$ & 6 & 21 & & & 1.05 & +3 & & & 4.30 & -7 & 7.24 & -6 & 9.06 & -7 & 2.03 & -4 \\
\hline $9-8-75$ & $9-18-75$ & 10 & 31 & & & 1.27 & +3 & & & $2 . S F$ & -7 & $? .84$ & -6 & 1.19 & -6 & 2.67 & -4 \\
\hline $9-18-75$ & $10-17-75$ & 29 & 60 & & & $5 . n ?$ & +2 & & & 1.13 & -7 & 3.86 & -7 & 1.31 & -6 & 2.92 & -4 \\
\hline $10-17-75$ & $11-18-75$ & 32 & 92 & & & 2.87 & +2 & & & 6.46 & -8 & 2.01 & -7 & 1.37 & -6 & 3.08 & -4 \\
\hline $11-18-75$ & $12-17-75$ & 29 & 121 & & & $7 . n 6$ & $+?$ & & & 1.50 & -7 & 5.44 & -7 & $1.6 ?$ & -6 & 3.63 & -4 \\
\hline $12-17-75$ & $1-15-76$ & 29 & 150 & & & 7.07 & $+?$ & & & 1.89 & -7 & 6.12 & -7 & $1.8 n$ & -6 & $n . n 4$ & -4 \\
\hline$r$ & & & & & & & & & & & & & & & & & \\
\hline & & & & & & & & & & & & & & & & & \\
\hline & & & & & & & & & & & & & & & & & \\
\hline & & & & & & & & & & & & & & & & & \\
\hline & & & & & & & & & & & & & & & & & \\
\hline & & & & & & & & & & & & & & & & & \\
\hline
\end{tabular}


LEACH TEST IDENTIFICATION NUMBER

$Z-5$

LABORATORY WHERE TESTS PERFORMED

ANALYST

DATE RESULTS REPORTS

Part C. Experimental Data

Constituent Analyzed. A

Curium-242

Initial Amount in Sample $A_{0}$

$1.867 \times 10^{-2} \mathrm{Ci}$

\begin{tabular}{|c|c|c|c|c|c|c|c|c|c|c|c|c|c|}
\hline \multicolumn{2}{|c|}{ TIME AND DATE } & \multirow{2}{*}{$i_{n}$} & \multirow{2}{*}{$\begin{array}{c}\text { TOTAL } \\
\mathrm{T}\end{array}$} & \multirow{2}{*}{$\begin{array}{l}\text { DAYS } \\
\text { DECAY. }\end{array}$} & \multirow{2}{*}{$\begin{array}{l}\text { DECAY } \\
\text { FACTOR }\end{array}$} & \multirow{2}{*}{$\begin{array}{c}\text { AS } \\
\text { ANALYZED } \\
(\mathrm{H} / \mathrm{m} / \mathrm{ml}) \cdots\end{array}$} & \multirow{2}{*}{$\begin{array}{c}\text { BLANK } \\
(\mathrm{d} / \mathrm{m} / \mathrm{ml})\end{array}$} & \multirow{2}{*}{$\begin{array}{l}\text { CORRECTED } \\
\text { CONC. } \\
1\end{array}$} & \multirow{2}{*}{\multicolumn{2}{|c|}{$a_{1} \cdots$}} & \multirow{2}{*}{$\frac{a_{1}}{A_{0}}\left(\frac{w}{s_{n}}\right)$} & \multirow{2}{*}{$\sum a_{1}$} & \multirow{2}{*}{$\frac{\sum_{\text {an }}^{\prime}}{A_{0}}$} \\
\hline IN & OUT & & & & & & & & & & & & \\
\hline $8-18-75$ & $8-19-75$ & 1 & 1 & 30 & 0.907 & $1.59+3$ & $<?$ & & 3.92 & -7 & & & \\
\hline $8-19-75$ & $8-20-75$ & 1 & 2 & 29 & 0.911 & $1.8+7$ & & & 4.45 & -9 & & & \\
\hline $8-20-75$ & $8-21-75$ & 1 & 3 & 28 & 0.914 & $0.4+1$ & & & 2.32 & -9 & & & \\
\hline $8-21-75$ & $8-22-75$ & 1. & 4 & 27 & 0.917 & $1.03+?$ & & & 2.53 & -8 & & & \\
\hline $8-22-75$ & $8-25-75$ & 3 & 7 & -24 & 0.926 & $? .79+?$ & & “ & 6.76 & -8 & & & \\
\hline $8-25-75$ & $9-2-75$ & 8 & 15 & 31 & 0.905 & $7.6+1$ & & & 1.89 & -8 & & & \\
\hline $9-2-75$ & $9-8-75$ & 6 & 21 & 36 & 0.923 & $5.85+?$ & & & 1.43 & -7 & & & \\
\hline $9-8-75$ & $9-18-75$ & 10 & 31 & 26 & 0.920 & $6 . n 2+?$ & & & 1.47 & -7 & & & \\
\hline $9-18-75$ & $10-17-75$ & 29 & 60 & 19 & 0.942 & $3.23+?$ & & & 7.72 & -8 & & & \\
\hline $10-17-75$ & $11-18-75$ & 32 & 92 & 196 & 0.449 & $7.36+1$ & & & 3.69 & -8 & & & \\
\hline $11-18-75$ & $12-17-75$ & 29 & 121 & 37 & 0.886 & $1.17+?$ & & & 2.97 & -8 & & & \\
\hline $12-17-75$ & $1-15-76$ & 29 & 150 & 8 & 0.975 & $3.5^{a}+?$ & & & 8.29 & -8 & & & \\
\hline$r$ & & & & & & & & & & & & & \\
\hline- & & & & & & & & & & & & & \\
\hline & & & & & & & & & & & & & \\
\hline & & & & & & & & & & & & & \\
\hline & & & & & & & & & & & & & \\
\hline & & & & & & & & & & & & & \\
\hline
\end{tabular}

- Concentration in leachate, show units.

- $a_{1}=$ curracted concentration $x V_{1} \times$ factorto convertio same units as $A_{2}$. 


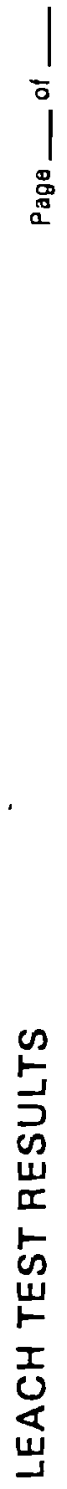

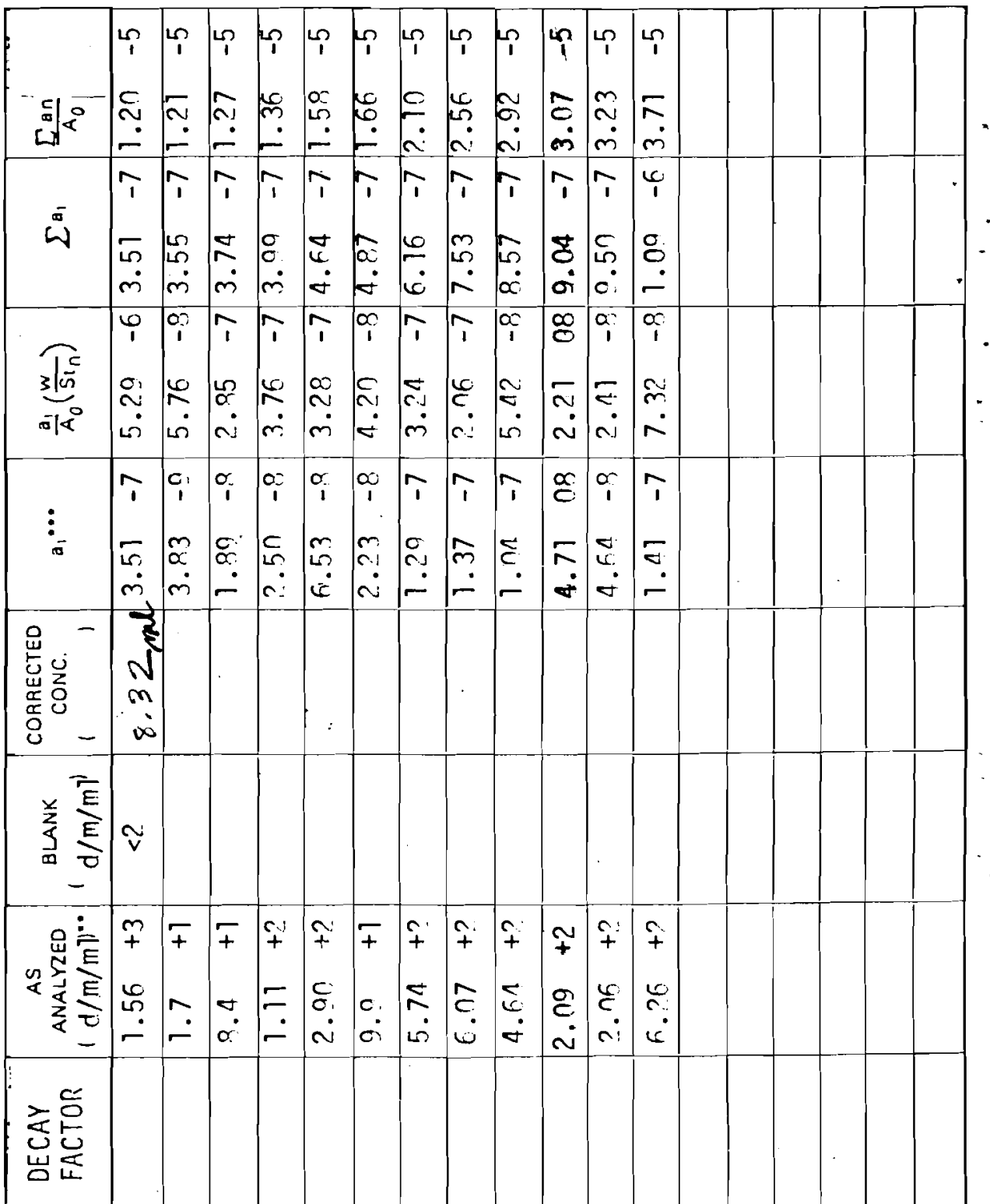

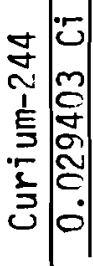

\section{吕出}

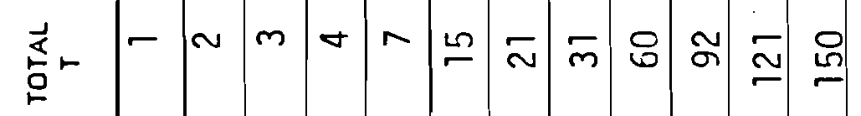

$$
=---m \infty \text { m }
$$


$\because$

LEACH TEST IDENTIFICATION NUMBER Z Z-5

LABORATORY WHERE TESTS PERFORMED

ANALYST

DATE RESULTS REPORTS

Part C. Experimental Data

Constituen: Analyzed, A Strontium-90 + Yttrium-90

Initial Amount in Sample Ao 1.3701

\begin{tabular}{|c|c|c|c|c|c|c|c|c|c|c|c|c|c|c|c|c|}
\hline \multicolumn{2}{|c|}{ IIME AND DATE } & \multirow{2}{*}{ in } & \multirow{2}{*}{$\underset{T}{\text { TOTAL }}$} & \multirow{2}{*}{$\begin{array}{l}\text { DAYS } \\
\text { DECAY }\end{array}$} & \multirow{2}{*}{$\begin{array}{l}\text { DECAY } \\
\text { FACTOR }\end{array}$} & \multirow{2}{*}{$\begin{array}{c}\text { AS } \\
\text { ANALYZED } \\
(\mathrm{d} / \mathrm{m} / \mathrm{ml})^{\circ}\end{array}$} & \multirow{2}{*}{$\begin{array}{l}\text { BLANK } \\
\mathrm{d} / \mathrm{m} / \mathrm{ml}^{\prime}\end{array}$} & \multirow{2}{*}{$\begin{array}{l}\text { CORRECTED } \\
\text { CONC. }\end{array}$} & \multirow{2}{*}{\multicolumn{2}{|c|}{$a_{1} \cdots$}} & \multirow{2}{*}{\multicolumn{2}{|c|}{$\frac{a_{1}}{A_{0}}\left(\frac{W}{S s_{n}}\right)$}} & \multirow{2}{*}{\multicolumn{2}{|c|}{$\sum a_{1}$}} & \multirow{2}{*}{\multicolumn{2}{|c|}{$\frac{\sum_{a n}}{A_{0}}$}} \\
\hline IN & OUT & & & & & & & & & & & & & & & \\
\hline $8-18-75$ & $8-19-75$ & 1 & 1 & & & $2 . n+6$ & & $V=8.33 \mathrm{ml}$ & $1.5^{n}$ & -4 & 1.46 & -4 & 4.57 & -1 & $3 . ? n$ & -4 \\
\hline $8-19-75$ & $8-20-75$ & 1 & 2 & & & $5.2+1$ & & $r=8.36$ & 1.17 & -5 & $3.7^{n}$ & $-f$ & 1.62 & -4 & 3.37 & -4 \\
\hline $8-20-75$ & $8-21-75$ & 1 & 3 & & & $4 . ?+1$ & & & 0.16 & -6 & $3 . n 5$ & -6 & $4.7 ?$ & -4 & 3.41 & -1 \\
\hline $8-27-75$ & $8-22-75$ & 1. & 4 & & & $5.7+1$ & & & $1.3 ?$ & -5 & 4.15 & -6 & $4.0,1$ & -1 & 3.54 & -4 \\
\hline $8-22-75$ & $8-25-75$ & 3 & 7 & & & $6.3+n$ & & . & $1.4 ?$ & -5 & 1.53 & -6 & 4.09 & -4 & 3.64 & -4 \\
\hline $8-25-75$ & $9-2-75$ & 8 & 15 & & & $3.5+5$ & & & 7.89 & -5 & 3.18 & -6 & 5.77 & -4 & 4.21 & -4 \\
\hline $9-2-75$ & $9-8-75$ & 6 & 21 & & & $6.3+5$ & & & $1.4 ?$ & -4 & 7.61 & -6 & 7.72 & -4 & 5.25 & -4 \\
\hline $9-8-75$ & $9-18-75$ & 10 & 31 & & & $6 . n+5$ & & & 1.35 & -4 & 1.37 & -6 & 8.54 & -4 & 6.24 & -4 \\
\hline $9-18-75$ & $10-17-75$ & 29 & 60 & & & $4.6+5$ & & & $7 . n 4$ & -1 & 1.15 & -6 & 0.58 & -4 & 6.99 & -4 \\
\hline $10-17-75$ & $11-18-75$ & 32 & 92 & & & $2 . n+5$ & & & 4.57 & -5 & 1.55 & -7 & $1 . n 0$ & -3 & $7.3 ?$ & -4 \\
\hline $11-18-75$ & $12-17-75$ & 29 & 121 & & & $5.8+5$ & & & 1.31 & -4 & 1.45 & -6 & 1.13 & -3 & 8.25 & -4 \\
\hline $12-17-75$ & $1-15-76$ & 29 & 150 & & & $7.2+5$ & & & $1.6 ?$ & -4 & 1.31 & -6 & 1.29 & -3 & 9.44 & -4 \\
\hline$r$ & & & & & & & & & & & & & & & & \\
\hline & & & & & & & & & & & & & & & & \\
\hline & & & & & & & & & & & & & & & & \\
\hline & & & & & & & & & & & & & & & & \\
\hline & & & & & & & & & & & & & & & & \\
\hline & & & & & & & & & & & & & & & & \\
\hline
\end{tabular}

-. Concentration in leachate, show units.

$\cdots_{B}=$ conracter conceneration $\times V$ x factor to convern to samo innits as $A_{0}$ 


\section{LEACH TEST RESULTS}

LEACH TEST IDENTIFICATION NUMBER _ Z Z-

LABORATORY WHERE TESTS PERFORMED Battelle-Northwest

ANALYST

DATE RESULTS REPORTED.

\section{Part A. Description of Leach Specimen}

Specimen ldentification Number Z-6

Proportion of Waste Incorporated in Mixlure__rradiated LWR fuel pellet__ Weight \%

or Volume\%

(Based on initial volumes)

Type of Waste, Chemical and Radioisotopic Composition, and Specific Activity of the Waste

Type and Composition of the Immobilization Material

Pieparaiion of Specimen

Storage Conditions

Appearance: 
LEACH TEST IDENTIFICATION NUMBER _ Z Z

LABORATORY WHERE TESTS PERFORMED Battelle-Northwest

ANALYST

DATE RESULTS REPORTED

\section{Part B. Description of Leach Test Procedure}

Sample Preparation*

Initial Weight of Sample, $W$

Volume of Sample, $V$

$12.53 \mathrm{~cm}^{2}$

Surface Area of Sample. $\mathrm{S}$
Method of Surface Area Determination_ geometric approximation

Diagram of Leach Apparaius:__ Paige type

Leachant Deionized water

$\begin{array}{ll}\text { Leachant Volume, } V_{L} & 500 \mathrm{ml} \\ \text { Sampling Procedure } & \text { drain leachant and refill at samplina }\end{array}$

Analytical Techniques:

$\mathrm{pH}$ Instrument Identification and Calibration

Constituent $a_{1}$, Analytical Procedure, Standard Deviation of Method

Constituent $\mathrm{a}_{2}$, Analytical Procedure, Standard Deviation of Method

*If different from "Preparation of Specimen" in Part A. 
$\because$

:ACH TEST IDENTIFICATION NUMBER Z-E

IBORATORY WHERE TESTS PERFORMED

VALYST .

ATE RESULTS REPORTS

C. Experimental Data

Constituent Analyzed, A Cesium-137

Initial Amount in Sample $A_{0} \quad 0.936$ ? Ci

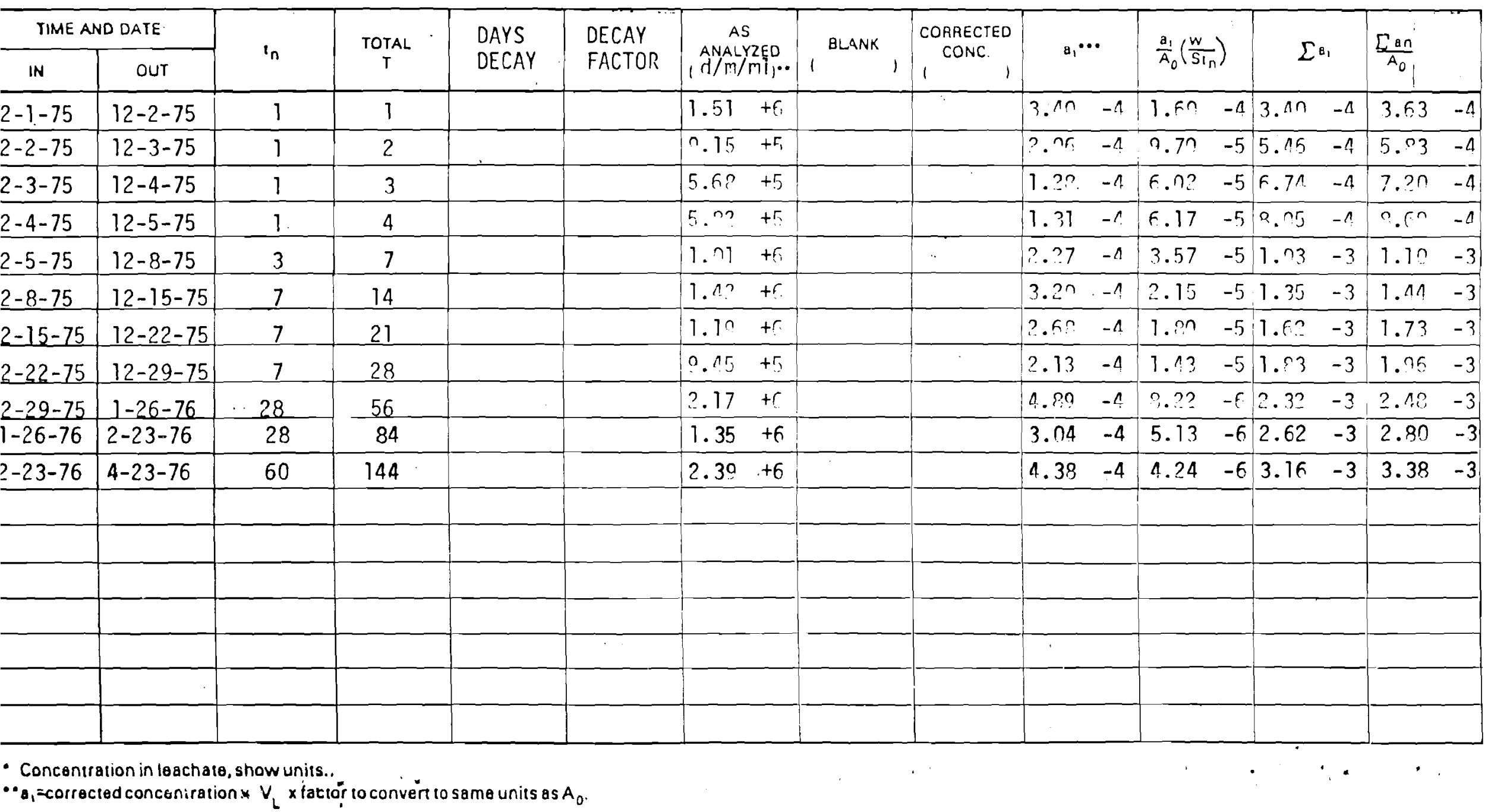


$\cdot$

ACH TEST IDENTIFICATION NUMBER

Z-6

IBORATORY WHERE TESTS PERFORMED

VALYST

ATE RESULTS REPORTS

nC. Experimental Data

Constituent Analyzed, A

Antimony-125

Initial Amount in Sample $A_{0}$

$3.7432 \times 10^{2} \mathrm{Ci}$

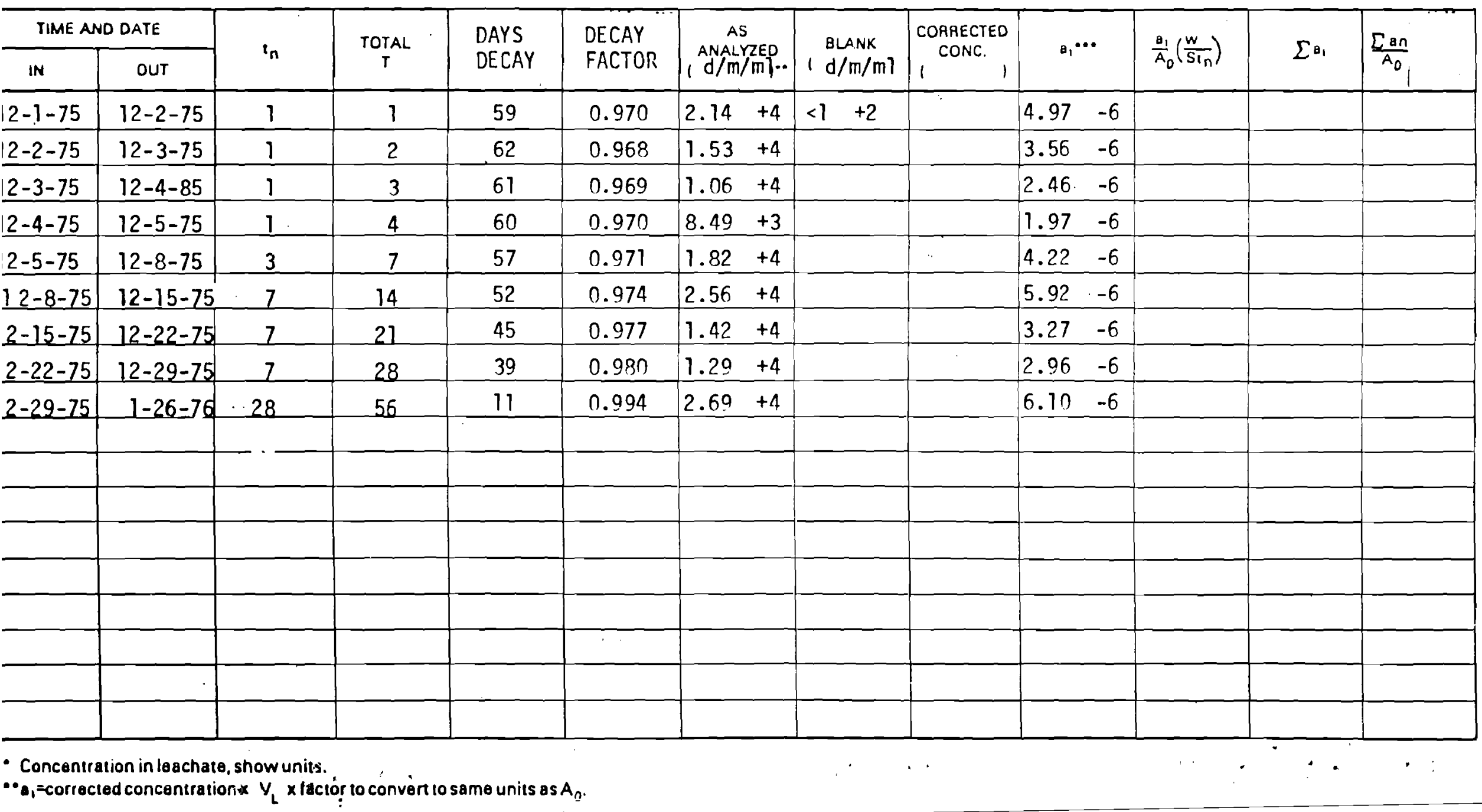


LEACH TEST IDENTIFICATION NUMBER

Z-6

LABORATORY WHERE TESTS PERFORMED

ANALYST

DATE RESULTS REPORTS

Part C. Experimental Data

Constituent Analyzed, A $\quad$ Plutonium-23a $+2.4 n$

Initial Amount in Sample $A_{0}-4.6918 \times 17^{-3} \mathrm{Ci}$

\begin{tabular}{|c|c|c|c|c|c|c|c|c|c|c|c|c|c|c|c|c|c|}
\hline \multicolumn{2}{|c|}{ TIME AND DATE } & \multirow{2}{*}{$i_{n}$} & \multirow{2}{*}{$\underset{T}{\text { TOTAL }}$} & \multirow{2}{*}{$\begin{array}{l}\text { DAYS } \\
\text { DECAY }\end{array}$} & \multirow{2}{*}{$\begin{array}{l}\text { DECAY } \\
\text { FACTOR }\end{array}$} & \multirow{2}{*}{\multicolumn{2}{|c|}{$\begin{array}{c}\text { AS } \\
\text { ANALYZED } \\
(\mathrm{d} / \mathrm{m} / \mathrm{ml}) . .\end{array}$}} & \multirow{2}{*}{\begin{tabular}{|c|}
$/ a_{.} T$ \\
BLANK \\
$(\mathrm{d} / \mathrm{m} / \mathrm{ml})$
\end{tabular}} & \multirow{2}{*}{$\begin{array}{l}\text { CORRECTED } \\
\text { CONC. } \\
1 \text { a } / \mathrm{T},\end{array}$} & \multirow{2}{*}{\multicolumn{2}{|c|}{$a_{1} \cdots$}} & \multirow{2}{*}{\multicolumn{2}{|c|}{$\frac{a_{1}}{A_{0}}\left(\frac{w}{S_{I_{n}}}\right)$}} & \multirow{2}{*}{\multicolumn{2}{|c|}{$\sum a_{1}$}} & \multirow{2}{*}{\multicolumn{2}{|c|}{$\frac{\sum_{\text {an }}}{A_{0}}$}} \\
\hline IN & OUT & & & & & & & & & & & & & & & & \\
\hline $12-1-75$ & $12-2-75$ & 11 & 1 & & & 2.26 & +3 & $1 . r^{3}-4$ & & 5.09 & -7 & 4.80 & -5 & 5.00 & -7 & 1.08 & -4 \\
\hline $12-2-75$ & $12-3-75$ & 11 & 2 & & & 6.97 & +2 & $\begin{array}{ll}3.3 & -5\end{array}$ & & 1.55 & -7 & 1.46 & -5 & 6.64 & -7 & 1.42 & -4 \\
\hline $12-3-75$ & $12-4-75$ & 11 & 3 & & & 5.05 & $+?$ & $2.4-5$ & & 1.11 & -7 & 1.07 & -5 & 7.77 & -7 & 1.66 & -4 \\
\hline $12-4-75$ & $12-5-75$ & 11 & 4 & & & 4.78 & +2 & $2.3-5$ & & 1.08 & -7 & 1.01 & -5 & 8.85 & -7 & 1.88 & -4 \\
\hline $12-5-75$ & $12-8-75$ & 36 & 7 & & & 1.08 & +3 & $5.2-5$ & $8.10-8$ & 2.43 & -7 & 7.64 & -6 & 1.13 & -6 & 2.40 & -4 \\
\hline $12-8-75$ & $12-15-75$ & 710 & 14 & & & 1.39 & +3 & $9.5 \quad-6$ & $4.44-8$ & 3.11 & -7 & 4.18 & -6 & 1.44 & -6 & $3 . \cap 6$ & -4 \\
\hline $12-15-75$ & $12-22-75$ & 714 & 21 & & & 7.84 & $+?$ & $5.4-6$ & $2.52-8$ & 1.77 & -7 & 2.36 & -6 & $1.6 ?$ & -6 & 3.44 & -4 \\
\hline $12-22-75$ & $12-29-75$ & 714 & 28 & & & 6.16 & $+?$ & $4.4-6$ & $2.08-8$ & 1.46 & -7 & 1.96 & -6 & 1.76 & -6 & 3.75 & -4 \\
\hline $12-29-75$ & $1-26-76$ & .28175 & 56 & & & 6.45 & +4 & $1.1-6$ & $5.18=7$ & 1.45 & -5 & 4.88 & -5 & 1.63 & -5 & 3.47 & -3 \\
\hline $1-26-76$ & $2-23-76$ & 2856 & 84 & & & 3.82 & +2 & $6.5-7$ & $3.07-9$ & 8.60 & -8 & 2.90 & -7 & 1.64 & -5 & 3.29 & -3 \\
\hline $2-23-76$ & $4-23-76$ & 60120 & 144 & & & 9.30 & +2 & $74-7$ & $3.48-9$ & 2.09 & -7 & 3.29 & -7 & 1.66 & -5 & 3.54 & -3 \\
\hline & & & & & & & & & & & & & & & & & \\
\hline & & & & & & & & & & & & & & & & & \\
\hline & & & & & & & & & & & & & & & & & \\
\hline & & & & & & & & & & & & & & & & & \\
\hline & & & & & & & & & & & & & & & & & \\
\hline & & & & & & & & & & & & & & & & & \\
\hline & & & & & & & & & & & & & & & & & \\
\hline
\end{tabular}

- Concentration in leachate, show units.

$\cdots_{B_{1}}=$ corrected concentration $\times V_{L} \times$ factor to convert to same units as $A_{Q}$ 
ACH TEST IDENTIFICATION NUMBER

$7-6$

IBORATORY WHERE TESTS PERFORMED

VALYST

ATE RESULTS REPORTS

in C. Experimental Data

Constituent Analyzed, A

Initial Amount in Sample $A_{0}$

Curium-242

$1.224 \times 10^{-2} \mathrm{Ci}$

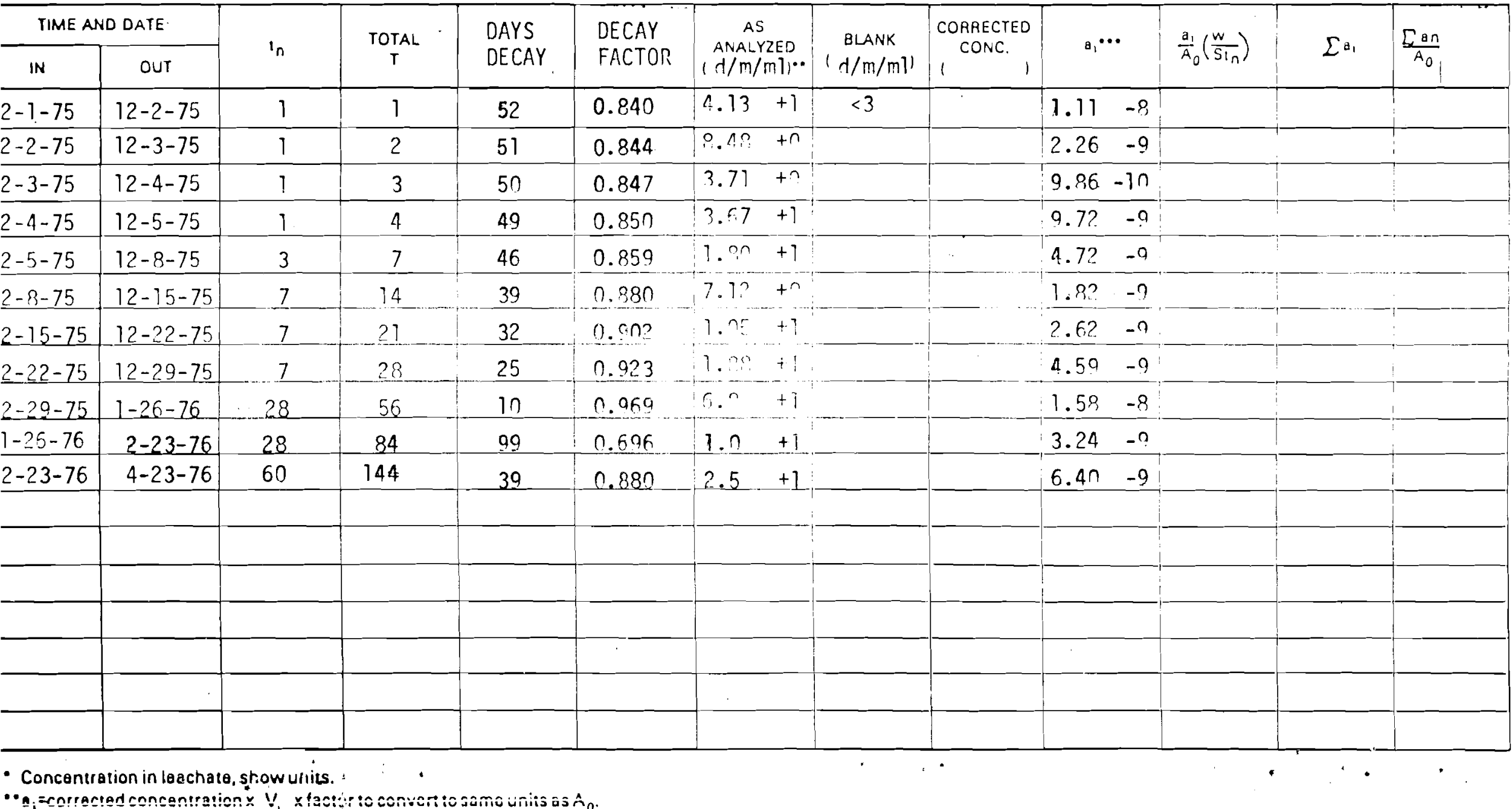


$\because$

EACH TEST IDENTIFICATION NUMBER

$Z-6$

ABORATORY WHERE TESTS PERFORMED

INALYST

JATE RESULTS REPORTS

'art C. Exporimental Data

Constituent Analyzed, A $\quad$ Curium-244

Initial Amount in Sample $A_{0} \quad 0.030863 \mathrm{Ci}$

\begin{tabular}{|c|c|c|c|c|c|c|c|c|c|c|c|c|c|c|c|c|}
\hline \multicolumn{2}{|c|}{ TIME AND DATE- } & \multirow{2}{*}{$I_{n}$} & \multirow{2}{*}{$\begin{array}{c}\text { TOTAL } \\
\mathrm{T}\end{array}$} & \multirow{2}{*}{$\begin{array}{l}\text { DAYS } \\
\text { DECAY }\end{array}$} & \multirow{2}{*}{$\begin{array}{l}\text { DECAY } \\
\text { FACTOR }\end{array}$} & \multirow{2}{*}{$\begin{array}{c}\text { AS } \\
\text { ANALYZED } \\
1 \mathrm{~d} / \mathrm{m} / \mathrm{m}\rceil)^{\circ}\end{array}$} & \multirow{3}{*}{$\frac{\begin{array}{c}\text { BLANK } \\
\mathrm{d} / \mathrm{m} / \mathrm{m}]^{\prime}\end{array}}{<3}$} & \multirow{2}{*}{$\begin{array}{l}\text { CORRECTED } \\
\text { CONC. } \\
1\end{array}$} & \multirow{2}{*}{\multicolumn{2}{|c|}{$a_{1} \cdots$}} & \multirow{2}{*}{\multicolumn{2}{|c|}{$\frac{a_{1}}{A_{0}}\left(\frac{w}{S_{n}}\right)$}} & \multirow{2}{*}{\multicolumn{2}{|c|}{$\sum a_{1}$}} & \multirow{2}{*}{\multicolumn{2}{|c|}{$\frac{\sum \text { an }}{A_{0}}$}} \\
\hline $\mathbb{I N}$ & OUT & & & & & & & & & & & & & & & \\
\hline $12-1-75$ & $12-2-75$ & 1 & 1 & & & $1.02+2$ & & & $2.3 n$ & -0 & 3.20 & -7 & 2.30 & -8 & 7.4 .4 & -7 \\
\hline $12-2-75$ & $12-3-75$ & 1 & 2 & & & $1.51+1$ & & & $3.4 n$ & -9 & 4.90 & -8 & $? . \overline{54}$ & -8 & 8.55 & -7 \\
\hline $12-3-75$ & $12-4-75$ & 1 & 3 & & & $6.4+n$ & & & 1.44 & $-?$ & $2 . n 7$ & -0 & 2.78 & -8 & 9.01 & -7 \\
\hline $12-4-75$ & $12-5-75$ & 1. & 4 & & & $5.43+1$ & & & $1.2 ?$ & -8 & 1.75 & -7 & $4 . n$ & -8 & $1.3 n$ & -6 \\
\hline $12-5-75$ & $12-8-75$ & 3 & 7 & & & $4.01+1$ & & . & 0.03 & -9 & $4.3 n$ & -8 & 4.91 & -3 & 1.50 & -6 \\
\hline $12-8-75$ & $12-15-75$ & 7 & 14 & & & $2.35+1$ & & & 6.12 & -3 & 1.32 & -8 & 5.55 & -8 & 1.80 & -6 \\
\hline $12-15-75$ & $12-22-75$ & 7 & 21 & & & $2.55+1$ & & & 5.74 & -0 & 1.18 & -8 & 6.12 & -8 & 1.38 & -6 \\
\hline $12-22-75$ & $12-29-75$ & 7 & 28 & & & $4.14+1$ & & & a. 32 & $-?$ & 1.91 & -8 & 7.76 & -8 & 2.29 & -6 \\
\hline $12-29-75$ & $1-26-76$ & .28 & 56 & & & $1.55+?$ & & & 3.49 & -0 & 1.79 & -9 & 1.05 & -7 & 3.42 & -6 \\
\hline $1-26-76$ & $2-23-76$ & 28 & 84 & & & $3.6+1$ & & & 8.11 & -9 & 4.15 & -9 & 1.13 & -7 & 3.66 & -6 \\
\hline $2-23-76$ & $4-23-76$ & 60 & 144 & & & $7.3+1$ & & & 1.64 & -8 & 3.92 & -9 & 1.29 & -7 & 4.10 & -6 \\
\hline & & & & & & & & & & & & & & & & \\
\hline & & & & & & & & & & & & & & & & \\
\hline & & & & & & & & & & & & & & & & \\
\hline & & & & & & & & & & & & & & & & \\
\hline & & & & & & & & & & & & & & & & \\
\hline & & & & & & & & & & & & & & & & \\
\hline
\end{tabular}

- Concentration in leachate, show units.

$\cdots a_{1}=$ corrected concentration $\times V_{L} \times$ factor to convert to same units as $A_{0}$. 
¿ACH TEST IDENTIFICATION NUMBER

$Z-6$

ABORATORY WHERE TESTS PERFORMED

NALYST

ATE RESULTS REPORTS

in C. Experimental Data

Constituent Analyzed, A

Am-241

Initial Amount in Sample $A_{0}$

$2.474 \times 11^{-3} \mathrm{CI}$

\begin{tabular}{|c|c|c|c|c|c|c|c|c|c|c|c|c|c|c|c|c|c|}
\hline \multicolumn{2}{|c|}{ TIME AND DATE } & \multirow{2}{*}{$i_{n}$} & \multirow{2}{*}{$\underset{T}{\text { TOTAL }}$} & \multirow{2}{*}{$\begin{array}{l}\text { DAYS } \\
\text { DECAY }\end{array}$} & \multirow{2}{*}{$\begin{array}{l}\text { DECAY } \\
\text { FACTOR }\end{array}$} & \multirow{2}{*}{\multicolumn{2}{|c|}{$\begin{array}{c}\text { AS } \\
\text { ANALYZED } \\
1 \mathrm{~d} / \mathrm{m} / \mathrm{m}\rceil . .\end{array}$}} & \multirow{2}{*}{$\begin{array}{l}\text { BLANK } \\
\mathrm{d} / \mathrm{m} / \mathrm{m} 1\end{array}$} & \multirow{2}{*}{$\begin{array}{l}\text { CORRECTED } \\
\text { CONC. } \\
1\end{array}$} & \multirow{2}{*}{\multicolumn{2}{|c|}{$a_{1} \cdots$}} & \multirow{2}{*}{\multicolumn{2}{|c|}{$\frac{a_{1}}{A_{0}}\left(\frac{w}{S i_{n}}\right)$}} & \multirow{2}{*}{\multicolumn{2}{|c|}{$\sum a_{1}$}} & \multirow{2}{*}{\multicolumn{2}{|c|}{$\frac{\sum_{\text {an }}}{A_{0}}$}} \\
\hline $\mathbb{I N}$ & OUT & & & & & & & & & & & & & & & & \\
\hline $12-1-75$ & $12-2-75$ & 1 & 1 & & & 5.6 & +1 & & & 1.26 & -8 & 2.25 & -6 & 1.26 & -8 & 5.10 & -6 \\
\hline $12-2-75$ & $12-3-75$ & 1 & 2 & & & 2.8 & +1 & & & 6.31 & -9 & 1.13 & -6 & 1.89 & -8 & 7.65 & -6 \\
\hline $12-3-75$ & $12-4-85$ & 1 & 3 & & & 6.5 & +1 & & & 1.46 & -8 & 2.61 & -6 & 3.36 & -8 & 1.36 & -5 \\
\hline $12-4-75$ & $12-5-75$ & 1 & 4 & & & 1.53 & +2 & & & 3.45 & -8 & 6.17 & -6 & 6.80 & -8 & 2.75 & -5 \\
\hline $12-5-75$ & $12-8-75$ & 3 & 7 & & & $2 . n$ & +1 & &. & 4.50 & -9 & 2.68 & -7 & 7.25 & -8 & 2.93 & -5 \\
\hline $12-8-75$ & $12-15-75$ & 7 & 14 & & & 3.1 & +1 & & & 6.98 & -9 & 1.78 & -7 & 7.95 & -8 & 3.21 & -5 \\
\hline $2-15-75$ & $12-22-75$ & 7 & $2]$ & & & 1.4 & +1 & & & 3.15 & -9 & $8 . n 5$ & -8 & 8.27 & -8 & 3.34 & -5 \\
\hline $2-22-75$ & $12-29-75$ & 7 & 28 & & & 2.2 & +1 & & & 4.96 & -9 & 1.27 & -7 & 8.76 & -8 & 3.54 & -5 \\
\hline $2-29-75$ & $1-26-76$ & 28 & 56 & & & 1.09 & +2 & & & 2.46 & -8 & 1.57 & -7 & 1.12 & -7 & 4.53 & -5 \\
\hline $1-26-76$ & $2-23-76$ & 28 & 84 & & & 5.9 & +1 & & & 1.33 & -8 & 8.50 & -8 & 1.25 & -7 & 5.07 & -5 \\
\hline $2-23-76$ & $4-23-76$ & 60 & 144 & & & $6 . n$ & +1 & & & 1.35 & -8 & $4 . n 2$ & -8 & 1.39 & -7 & 5.62 & -5 \\
\hline & & & & & & & & & & & & & & & & & \\
\hline & & & & & & & & & & & & & & & & & \\
\hline & & & & & & & & & & & & & & & & & \\
\hline & & & & & & & & & & & & & & & & & \\
\hline & & & & & & & & & & & & & & & & & \\
\hline & & & & & & & & & & & & & & & & & \\
\hline
\end{tabular}

- Concentration in leachate, show units.

- $a_{1}=$ corrected concentrationx $V_{b}$ x factor to convërn to same units as $A_{0}$. 


\section{LEACH TEST RESULTS}

\section{LEACH TEST IDENTIFICATION NUMBER Z Z-7 LABORATORY WHERE TESTS PERFORMED Battelle-Northwest}

ANALYST

DATE RESULTS REPORTED

Part A. Description of Leach Specimen

Specimen Identification Number Z Z -7

Proportion of Waste Incorporated in Mixture_ Irradiated LWR fuel pellet Weight \%

or Volume\%

(Based on initial volumes)

Type of Waste, Chemical and Radioisotopic Composition, and Specific Activity of the Waste

Type and Composition of the Immobilization Material

\section{Preparation of Specimen}

Storage Conditions

Appearance: 


\section{LEACH TEST IDENTIFICATION NUMBER Z Z-7 LABORATORY WHERE TESTS PERFORMED Battelle-Northwest} ANALYST

\section{DATE RESULTS REPORTED}

\section{Part B. Description of Leach Test Procedure}

Sample Preparation*

Initial Weight of Sample, $W \quad 4.4313 \mathrm{gm}$

Volume of Sample, $\mathrm{V}$

Surface Area of Sample, s_ $1 n, ?] \mathrm{cm}^{\text {? }}$

Method of Surface Area Determination_aeometric approximation

Diagram of Leach Apparatus:

Leachant Deionized water

Leachant Volume, $v_{1} \quad 500 \mathrm{ml}$

Sampling Procedure drain leachant and refill after samplina

Analytical Techniques:

$\mathrm{pH}$ Iristrument Identification and Calibration

Constituent $a_{1}$, Analytical Procedure, Standard Deviation of Method

Constituent $\mathrm{a}_{2}$. Analytical Procedure, Standard Deviation of Method

"If different from "Preparation of Specimen" in Part A. 
LEACH TEST IDENTIFICATION NUMBER

LABORATORY WHERE TESTS PERFORMED

ANALYST

DATE RESULTS REPORTS

Part C. Experimental Data

Constituent Analyzed, A Cesium-137

Initial Amount in Sample $A_{0} \underline{0.7452 ~ C i}$

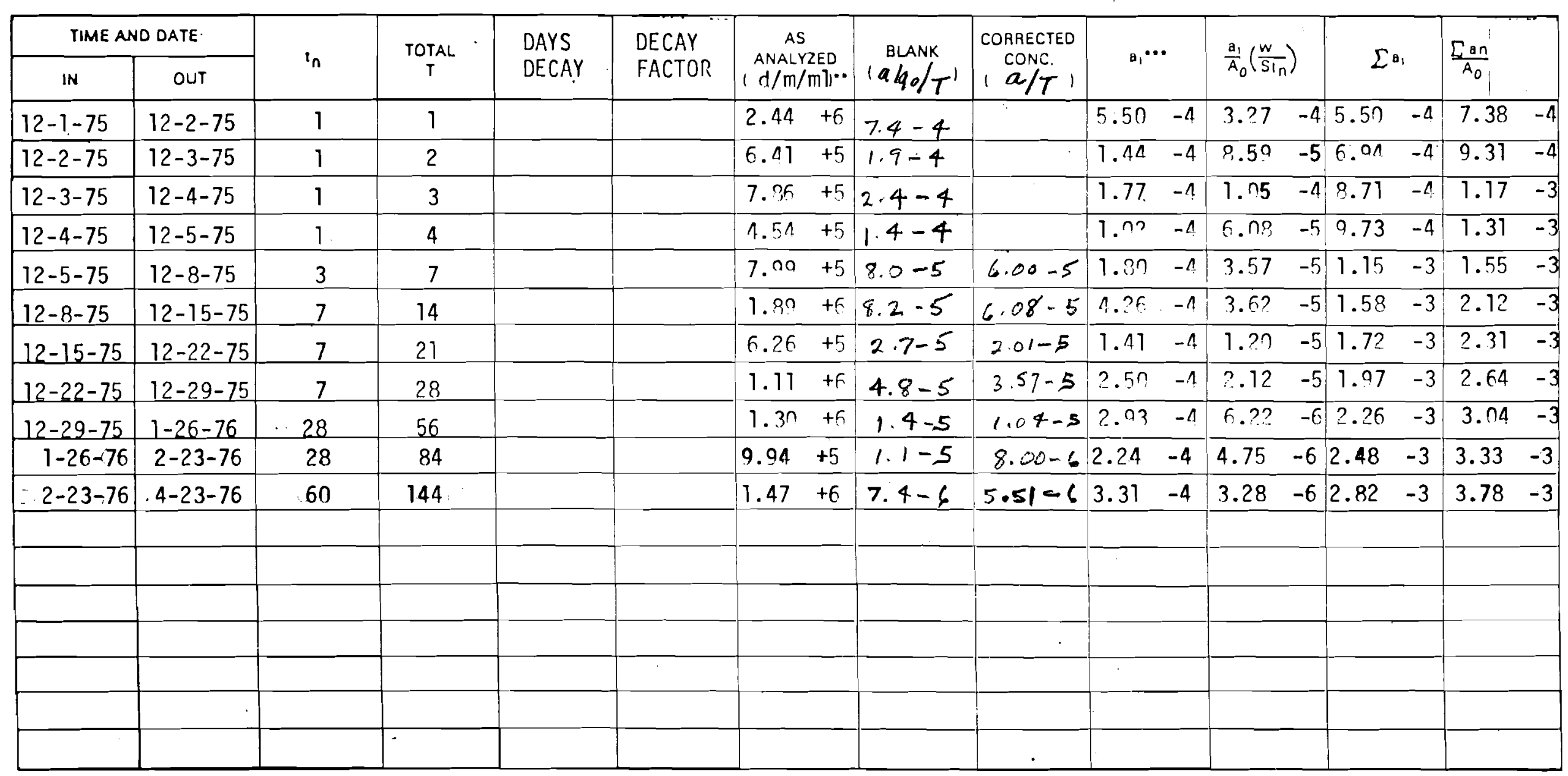

- Concentration in leachate, show units.

- $a_{1}=$ corrected concentration $\times V_{b} \times$ factor to convert to same units as $A_{0}$ 
EACH TEST IDENTIFICATION NUMBER

\section{ABORATORY WHERE TESTS PERFORMED}

INALYST

IATE RESULTS REPORTS

\section{art C. Experimental Data}

Constituent Analyzed, A $\longrightarrow$ Cesium-134

Initial Amount in Sample $A_{0}-1.0277 \mathrm{Ci}$

\begin{tabular}{|c|c|c|c|c|c|c|c|c|c|c|c|c|}
\hline \multicolumn{2}{|c|}{ TIME AND DATE } & \multirow{2}{*}{$i^{n}$} & \multirow{2}{*}{$\underset{T}{\text { TOTAL }}$} & \multirow{2}{*}{$\begin{array}{l}\text { DAYS } \\
\text { DECAY }\end{array}$} & \multirow{2}{*}{$\begin{array}{l}\text { DECAY } \\
\text { FACTOR }\end{array}$} & \multirow{2}{*}{$\begin{array}{c}\text { AS } \\
\text { ANALYZED } \\
1 \mathrm{~d} / \mathrm{m} / \mathrm{m}\rceil . .\end{array}$} & \multirow{2}{*}{$\begin{array}{l}\text { BLANK } \\
1 \mathrm{~d} / \mathrm{m} / \mathrm{m} 1\end{array}$} & \multirow{2}{*}{$\begin{array}{l}\text { CORAECTED } \\
\text { CONC. } \\
1\end{array}$} & \multirow{2}{*}{$a_{1} \cdots$} & \multirow{3}{*}{$\frac{a_{1}}{A_{0}}\left(\frac{w}{S i_{n}}\right)$} & \multirow{3}{*}{$\sum a_{1}$} & \multirow{2}{*}{$\frac{\Gamma_{\text {an }}}{A_{0}}$} \\
\hline IN & OUT & & & & & & & & & & & \\
\hline $12-1-75$ & $12-2-75$ & 1 & 1 & 51 & 0.966 & $2 . n 3+6$ & $6.4+3$ & & $4.73-4$ & & & \\
\hline $12-2-75$ & $12-3-75$ & 1 & 2 & 59 & 0.961 & $5.43+5$ & & & $1.27-4$ & & & \\
\hline $12-3-75$ & $12-4-85$ & 1 & 3 & 58 & 0.961 & $6.44+5$ & & & 1.51. -4 & & & \\
\hline $12-4-75$ & $12-5-75$ & 1 & 4 & 57 & 0.962 & $3.8 n+5$ & & & $8.9 n-5$ & & & \\
\hline $12-5-75$ & $12-8-75$ & 3 & 7 & 54 & 0.964 & $6.50+5$ & & $\therefore$ & $1.52 \quad-4$ & & & \\
\hline $12-8-75$ & $12-15-75$ & 7 & 14 & 47 & 0.969 & $1.6 n+6$ & & & $3.72-4$ & & & \\
\hline $12-15-75$ & $12-22-75$ & 7 & 21 & 40 & 0.973 & $4.94+5$ & & & $1.14 \quad-4$ & & & \\
\hline $12-22-75$ & $12-29-75$ & 7 & 28 & 33 & 0.978 & $9.88+5$ & & & $2.0 n \quad-4$ & & & \\
\hline $12-29-75$ & $1-26-76$ & 28 & 56 & 11 & 0.993 & $1.03+6$ & & & $2.34 \quad-4$ & & & \\
\hline $1-26-76$ & $2-23-76$ & 28 & 84 & 79 & 0.947 & $7.25+5$ & & & $1.72-4$ & & & \\
\hline $2-23-76$ & $4-23-76$ & 60 & 144 & 19 & 0.987 & $1.07+6$ & & & $2.44 \quad-4$ & & & \\
\hline & & & & & & & & & & & & \\
\hline & & & & & & & & & & & & \\
\hline & & & & & & & & & & & & \\
\hline & & & & & & & & & & & & \\
\hline & & & & & & & & & & & & \\
\hline & & & & & & & & & & & & \\
\hline
\end{tabular}


$\because$

LEACH TEST IDENTIFICATION NUMBER

LABORATORY WHERE TESTS PERFORMED

ANALYST

DATE RESULTS REPORTS

\section{Part C. Experimental Data}

Constituent Analyzed, A $\quad$ Curium-244

Initial Amount in Sample $A_{0}-0.024565 \mathrm{Ci}$

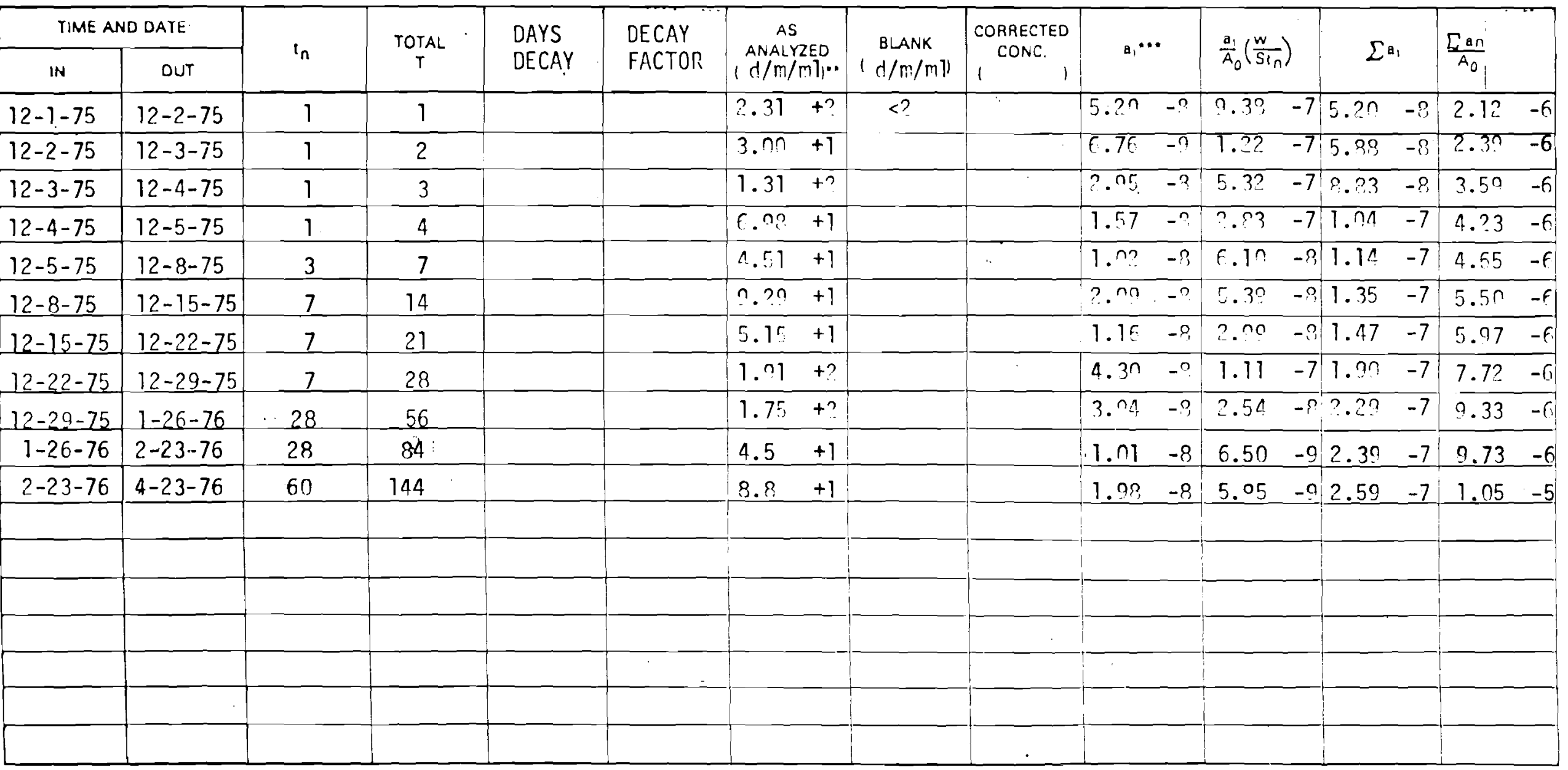

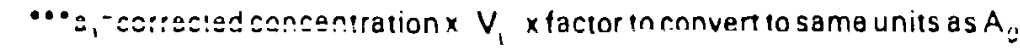


.EACH TEST IDENTIFICATION NUMBER

$2-7$

ABORATORY WHERE TESTS PERFORMED

ANALYST

DATE RESULTS REPORTS

sart C. Experimental Data

Constituent Analyzed, A

Antimony- 125

Initial Amount in Sample $A_{0}$

$2.379 \times 10^{-2} \mathrm{Ci}$

\begin{tabular}{|c|c|c|c|c|c|c|c|c|c|c|c|c|c|}
\hline \multicolumn{2}{|c|}{ TIME AND DATE } & \multirow{2}{*}{$t_{n}$} & \multirow{2}{*}{$\underset{T}{\text { TOTAL }}$} & \multirow{2}{*}{$\begin{array}{l}\text { DAYS } \\
\text { DECAY }\end{array}$} & \multirow{2}{*}{$\begin{array}{l}\text { DECAY } \\
\text { FACTOR }\end{array}$} & \multirow{2}{*}{\multicolumn{2}{|c|}{$\begin{array}{c}\text { AS } \\
\text { ANALYED } \\
1 \mathrm{~d} / \mathrm{m} / \mathrm{m} 1 . .\end{array}$}} & \multirow{2}{*}{$\begin{array}{l}\text { BLANK } \\
1 \mathrm{~d} / \mathrm{m} / \mathrm{ml}\end{array}$} & \multirow{2}{*}{$\begin{array}{c}\text { CORAECTED } \\
\text { CONC. } \\
1\end{array}$} & \multirow{2}{*}{$a_{1} \cdots$} & \multirow{2}{*}{$\frac{a_{1}}{A_{0}}\left(\frac{w}{S_{i_{n}}}\right)$} & \multirow{2}{*}{$\sum a_{1}$} & \multirow{2}{*}{$\frac{\sum \text { an }}{A_{0}}$} \\
\hline IN & our & & & & & & & & & & & & \\
\hline $12-1-75$ & $12-2-75$ & 1 & 1 & 59 & 0.970 & 2.12 & +4 & $<1+2$ & & $4.32-6$ & & & \\
\hline $12-2-75$ & $12-3-75$ & 1 & 2 & 62. & 0.968 & $1.2 n$ & +1 & & & $2.79-6$ & & & \\
\hline $12-3-75$ & $12-4-85$ & 1 & 3 & 61 & $n . n \in 0$ & 0.24 & +3 & & & $2.15-6$ & & & \\
\hline $12-4-75$ & $12-5-75$ & 1 & 4 & 60 & 0.970 & 7.64 & +3 & & & $1.77 \quad-6$ & & & \\
\hline $12-5-75$ & $12-8-75$ & 3 & 7 & 59 & 0.979 & $1.8 n$ & $+a$ & & .. & $0.19-6$ & & & \\
\hline $12-8-75$ & $12-15-75$ & 7 & 14 & 52 & 0.074 & 2.53 & +4 & & & $5.05-6$ & & & \\
\hline $12-15-75$ & $12-22-75$ & 7 & 21 & 45 & 0.077 & 9.7 & +3 & & & $2.21 \quad-6$ & & & \\
\hline $12-22-75$ & $12-29-75$ & 7 & 28 & 39 & $n .98 n$ & 1.26 & +4 & & & $? a n-6$ & & & \\
\hline $12-29-75$ & $1-26-76$ & 28 & 56 & 11 & 0.094 & 7.69 & +4 & & & $3.83-6$ & & & \\
\hline & & & & & & & & & & & & & \\
\hline & & & & & & & & & & & & & \\
\hline & & & & & & & & & & & & & \\
\hline & & & & & & & & & & & & & \\
\hline & & & & & & & & & & & & & \\
\hline & & & & & & & & & & & & & \\
\hline & & & & & & & & & & & & & \\
\hline & & & & & & & & & & & & & \\
\hline & & & & & & & & & & & & & \\
\hline
\end{tabular}




\begin{tabular}{|c|c|c|c|c|c|c|c|c|c|c|c|c|c|c|c|c|}
\hline TIME AN & D DATE & 1 & TOTAL & DAYS & DECAY & & ANK & CORRECTED & .. & & $\left(\frac{w}{w}\right)$ & & $\Gamma$ & & Ean & \\
\hline IN & OUT & n & $T$ & DECAY & FACTOR & $\mathrm{d} / \mathrm{m} / \mathrm{ml})^{\prime}$ & ${ }^{\prime} \mathrm{d} / \mathrm{m} / \mathrm{ml}^{\prime}$ & & $a_{1}$ & & $\bar{\theta}_{0} \sqrt{\sin n}$ & & $2^{\circ}$ & & $\overline{A_{0}}$ & \\
\hline $12-1-75$ & $12-2-75$ & 1 & 1 & & & $3.8+5$ & $9.9+3$ & & 8.56 & -5 & 3.31 & -5 & 8.56 & -5 & 7.48 & -5 \\
\hline $12-2-75$ & $12-3-75$ & 1 & 2 & & & $1.1+5$ & & & $2.4 ?$ & -5 & 0.50 & -6 & $1.1 ?$ & -4 & 9.64 & -5 \\
\hline $12-3-75$ & $12-4-75$ & 1 & 3 & & & $1.1+5$ & & & 2.43 & -5 & 9.59 & -6 & 1.35 & -4 & 1.18 & -4 \\
\hline $12-4-75$ & $12-5-75$ & 1 & 4 & & & $1.7++5$ & & & 3.93 & -5 & 1.48 & -5 & 1.73 & -4 & 1.52 & -4 \\
\hline $12-5-75$ & $12-8-75$ & 3 & 7 & & & $0.3+4$ & & . & $? .00$ & -5 & $2.7 n$ & -6 & 1.04 & -4 & 1.70 & -4 \\
\hline $12-8-75$ & $12-15-75$ & 7 & 14 & & & & & & & & & & & & & \\
\hline $12-15-75$ & $12-22-75$ & 7 & 21 & & & $1.5+5$ & & & $3.3 ?$ & -5 & 1.87 & -6 & 2.62 & -4 & 2.20 & -4 \\
\hline $12-22-75$ & $12-29-75$ & 7 & 28 & & & $7.0+4$ & & & 1.50 & -5 & 0.71 & -7 & 2.78 & -4 & 2.43 & -4 \\
\hline $12-29-75$ & $1-26-76$ & 28 & 56 & & & $4.8+5$ & & & $1.0 ?$ & -4 & $1.4 ?$ & -6 & 3.86 & -4 & 3.37 & -4 \\
\hline $17-26-76$ & $2-23-76$ & 28 & 34 & & & $5.42+5$ & & & 1.22 & -4 & 1.68 & -6 & 5.08 & -4 & $A .44$ & -4 \\
\hline $2-23-76$ & $-4-23-76$ & 660 & 144 & & & $7.54+5$ & & & $1.7 n$ & -4 & $.1 .1 n$ & -6 & 6.78 & -4 & 5.92 & -4 \\
\hline & & & & & & & & & & & & & & & & \\
\hline & & & & & & & & & & & & & & & & \\
\hline & & & & & & & & & & & & & & & & \\
\hline & & & & & & & & & & & & & & & & \\
\hline & & & & & & & & & & & & & & & & \\
\hline & & & & & & & & & & & & & & & & \\
\hline & & & & & & & & & & & & & & & & \\
\hline
\end{tabular}


LABORATORY WHERE TESTS PERFORMED

ANALYST

DATE RESULTS REPORTS

Part C. Experimental Data

Constituent Analyzed, A

Cerium- 144

Initial Amount in Sample $A_{0}$

$0.8974 \mathrm{Ci}$

\begin{tabular}{|c|c|c|c|c|c|c|c|c|c|c|c|c|}
\hline \multicolumn{2}{|c|}{ TIME AND DATE } & \multirow{2}{*}{$t_{n}$} & \multirow{2}{*}{$\underset{T}{\text { TOTAL }}$} & \multirow{2}{*}{$\begin{array}{l}\text { DAYS } \\
\text { DECAY }\end{array}$} & \multirow{2}{*}{$\begin{array}{l}\text { DECAY } \\
\text { FACTOR }\end{array}$} & \multirow{2}{*}{$\begin{array}{c}\text { AS } \\
\text { ANALYZED } \\
1 \mathrm{~d} / \mathrm{m} / \mathrm{m}\} . .\end{array}$} & \multirow{2}{*}{$\begin{array}{l}\text { BLANK } \\
\mathrm{d} / \mathrm{m} / \mathrm{ml}\end{array}$} & \multirow{2}{*}{$\begin{array}{c}\text { CORAECTED } \\
\text { CONC. } \\
1\end{array}$} & \multirow{2}{*}{$a_{1} \cdots$} & \multirow{2}{*}{$\frac{a_{1}}{A_{0}}\left(\frac{w}{S I_{n}}\right)$} & \multirow{2}{*}{$\sum a_{1}$} & \multirow{2}{*}{$\frac{\sum \text { an }}{A_{0}}$} \\
\hline IN & OUT & & & & & & & & & & & \\
\hline $12-1-75$ & $12-2-75$ & 1 & 1 & 59 & 0.896 & $4.57+3$ & & & $1.15-6$ & & & \\
\hline $12-2-75$ & $12-3-75$ & 1 & 2 & 62 & 0.891 & $2.67+3$ & & & $6.75 \quad-7$ & & & \\
\hline $12-3-75$ & $12-4-85$ & 1 & 3 & 61 & 0.893 & $9.01+3$ & & & $2.27 \quad-6$ & & & \\
\hline $12-4-75$ & $12-5-75$ & 1 & 4 & 60 & 0.895 & $4.68+3$ & & & $1.18 \quad-6$ & & & \\
\hline $12-5-75$ & $12-8-75$ & 3 & 7 & 59 & 0.896 & $5.22+3$ & & 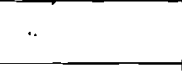 & $1.31-6$ & & & \\
\hline $12-8-75$ & $12-15-75$ & 7 & 14 & 52 & 0.909 & $4.97+3$ & & & $1.23 .-6$ & & & \\
\hline $12-15-75$ & $12-22-75$ & 7 & 21 & 45 & 0.921 & $<7.23+3$ & & & $\begin{array}{ll}3 . n 1 & -7\end{array}$ & & & \\
\hline $12-22-75$ & $12-29-75$ & 7 & 28 & 39 & 7.932 & $1.14+1$ & & & $2.75 \quad-6$ & & & \\
\hline $12-29-75$ & $1-26-76$ & $\cdots 28$ & 56 & 11 & 0.987 & $1.1 n+4$ & & & $2.53-6$ & & & \\
\hline & & & & & & & & & & & & \\
\hline & & & & & & & & & & & & \\
\hline & & & & & & & & & & & & \\
\hline & & & & & & & & & & & & \\
\hline & & & & & & & & & & & & \\
\hline & & & & & . & & & & & & & \\
\hline & & & & & & & & & & & & \\
\hline & & & & & & & & . & & & & \\
\hline
\end{tabular}

- Concentration in leachato, show units.

- $a_{a}=$ corrocted concentration $\times V_{L} \times$ factor to convert to same units as $A_{0}$. 


\section{C. Experimental Data}

Constituent Analyzed, A Ruthen i um-106

Initial Amount in Sample $A_{0}-0.6504 C$ C

\begin{tabular}{|c|c|c|c|c|c|c|c|c|c|c|c|c|}
\hline \multicolumn{2}{|c|}{ TIME AND DATE } & \multirow{2}{*}{$t_{n}$} & \multirow{2}{*}{$\underset{T}{\text { TOTAL }}$} & \multirow{2}{*}{$\begin{array}{l}\text { DAYS } \\
\text { DECAY }\end{array}$} & \multirow{2}{*}{$\begin{array}{l}\text { DECAY } \\
\text { FACTOR }\end{array}$} & \multirow{2}{*}{$\begin{array}{c}\text { AS } \\
\text { ANALYZED } \\
\mathrm{l} \mathrm{d} / \mathrm{m} / \mathrm{m}\} . .\end{array}$} & \multirow{2}{*}{$\begin{array}{l}\text { BLANK } \\
1 \mathrm{~d} / \mathrm{m} / \mathrm{m}\}\end{array}$} & \multirow{2}{*}{$\begin{array}{l}\text { CORRECTED } \\
\text { CONC. } \\
1\end{array}$} & \multirow{2}{*}{$a_{1} \cdots$} & \multirow{2}{*}{$\frac{a_{1}}{A_{0}}\left(\frac{w}{S_{I_{n}}}\right)$} & \multirow{2}{*}{$\sum a_{1}$} & \multirow{2}{*}{$\frac{\sum_{\text {an }}}{A_{0}}$} \\
\hline IN & out & & & & & & & & & & & \\
\hline$?-1-75$ & $12-2-75$ & 1 & 1 & 59 & 0.920 & $2.21+4$ & & & $5.41-6$ & & & \\
\hline$-2-75$ & $12-3-75$ & 1 & 2 & 62 & 0.916 & $1.66+4$ & & & $4.08 \quad-6$ & & & \\
\hline$-3-75$ & $12-4-85$ & 1 & 3 & 61 & 0.917 & $9.69+3$ & & & $2.38-6$ & & & \\
\hline$-4-75$ & $12-5-75$ & 1 & 4 & 60 & 0.918 & $6.74+3$ & & & $1.65-6$ & & & \\
\hline$-5-75$ & $12-8-75$ & 3 & 7 & 59 & 0.920 & $6.51+3$ & & $\therefore$ & $1.59-6$ & & & \\
\hline $2-8-75$ & $12-15-75$ & 7 & 14 & 52 & 0.929 & $5.89+3$ & & & $1.43 .-6$ & & & \\
\hline$-15-75$ & $12-22-75$ & 7 & 21 & 45 & 0.939 & $2.46+3$ & & & $\begin{array}{lll}5.90 & -7\end{array}$ & & & \\
\hline$-22-75$ & $12-29-75$ & 7 & 28 & 39 & 0.947 & $4.01+3$ & & & $\begin{array}{lll}9.54 & -7 \\
\end{array}$ & & & \\
\hline$-29-75$ & $1-26-76$ & 28 & 56 & 11 & 0.985 & $2.58+3$ & & & $5.90 \quad-7$ & & & \\
\hline & & & & & & & & & & & & \\
\hline & & & & & & & & & & & & \\
\hline & & & & & & & & & & & & \\
\hline & & & & & & & & & & & & \\
\hline & & & & & & & & & & & & \\
\hline & & & & & & & & & & & & \\
\hline & & & & & & & & & & & & \\
\hline & & & & & & & & & & & & \\
\hline & & & & & & & & & & & & \\
\hline
\end{tabular}


DISTRIBUTION

No. of

Copies

OFFSITE

UNITED STATES

1 ERDA Chicago Patent Group

9800 South Cass Avenue

Argonne, IL 60439

A. A. Churm

2 NRC Directorate of Licensing

for Fuels and Materials

4915 St. Elmo Avenue

Bethesda, MD 20014

Deputy Director for Fuels

and Materials

S. H. Smiley

Chief, Technical Support Branch

for Fuels and Materials

R. B. Chitwood

2 NRC Division of Materials and Fuel Cycle Facility Licensing Washington, DC 20555

Chief, Waste Management Branch

W. P. Bishop

J. S. Parry

1 ERDA Division of Biomedical and Environmental Research

Earth Sciences Branch

Washington, DC 20545

W. G. Belter

1 ERDA Division of Environmenta]

Control Technology

Washington, DC 20545

W. E. Mott
No. of

Copies
1 ERDA Division of Physical Research Washington, DC 20545

D. W. Readey

9 ERDA Division of Nuclear Fuel Cycle and Production Washington, DC

F. P. Baranowski

C. R. Cooley

G. H. Daly

W. K. Eister

0 . P. Gormley

C. Kuhlman

A. F. Perge

R. W. Ramsey

R. D. Walton

1 ERDA Idaho Operations Office P.0. Box 2108 Idaho Falls, ID 83401

K. K. Kennedy

1 ERDA Oak Ridge Operations Office P.0. Box X

Oak Ridge, TN 37830

E. H. Hardison

1 ERDA Savannah River Operations Office

P.0. Box A

Aiken, SC 29801

R. L. Chandler

27 ERDA Technical Information Center 
No. of

Copies

2 Allied Chemical Corporation

550 - 2nd Street

Idaho Falls, ID 83401

J. A. Buckham

B. R. Dickey

3 Allied-General Nuclear Services

P.0. Box 847

Barnwell, SC 29812

W. L. Godfrey

W. J. Price

c/o Bechtel Corporation

50 Beale Street

San Francisco, CA 94108

R. J. Cholister

1 Argonne National Laboratory

9700 South Cass Avenue

Argonne, IL 60439

M. J. Steindler

1 Bechtel Corporation

50 Beale Street

San Francisco, CA 94108

1 Brookhaven National Laboratory

Research Library, Reference Section Information Division

Upton, Long Island, NY 11973

M. Steinberg

1 C-E Refractories

Box 828

Valley Forge, PA 19482

A. W. Allen

1 Combustion Engineering, Inc.

Combustion Division

Windsor, CT 06095

R. Beekmann
No. of

Copies

3 Corning G1ass works

Technical Staffs Division

Corning, NY 14830

M. G. Britton

1 Dow Chemical Company (ERDA)

Rocky Flats Division

P.0. Box 888

Golden, C0 80401

D. L. Ziegler

4 E. I. du Pont de Nemours and Co.

Savannah River Laboratory

Aiken, SC 29801

R. F. Bradley

C. H. Ice

A. S. Jennings

J. A. Kelley

11 Electric Power Research Institute $3412 \mathrm{Hillview} \mathrm{Avenue}$

P.0. Box 10412

Palo Alto, CA 94304

R. F. Williams

2 Environmental Protection Agency Technology Assessment Division (AW-559)

Office of Radiation Programs Washington, DC 20460

G. L. Meyer

3 Exxon

RichTand, WA 99352

S. J. Beard

L. T. Lakey

M. E. Spaeth

2 General Electric Company

175 Curtner Avenue

(M/C 160)

San Jose, CA 95125

R. G. Barnes

A. Carson 
No. of

Copies

2 General Atomic Company

P.0. Box 81608

San Diego, CA 92138

L. H. Brooks

J. J. Shefcik

4 Dak Ridge National Laboratory

(ERDA)

Central Research Library

Document Reference Section Central Research Library, ORNL Laboratory Records Dept., ORNL Laboratory Records Dept., ORNL-RC P.0. Box X

Oak Ridge, TN 37830

1 Los Alamos Scientific Laboratory (ERDA)

P.0. Box 1663

Los Alamos, NM 87544

G. Cowens

2 Nuclear Fuel Services, Inc.

P.0. Box 124

West Valley, NY 14171

J. P. Duckworth, Plant Manager

6000 Executive Blvd., Suite 600

Rockville, MD 20852

W. Lewis, Vice President

1 Pennsylvania State University Materials Research Laboratory University Park, PA 16802

G. J. McCarthy

3 Sandia Laboratories

Albuquerque, NM 87107

R. W. Lynch

W. Weart

P. D. O'Brien
No. of

Copies

2 Union Carbide Corporation (HNL) Chemical Technology Division P.0. Box $Y$

Oak Ridge, TN 37830

J. 0. Blomeke

H. W. Godbee

1 Union Carbide Corporation (OWI) Office of Waste Isolation

P.0. Box $Y$

Oak Ridge, TN 37830

C. D. Zerby

1 University of Arizona

Department of Nuclear Engineering Tucson, AZ 85721

Roy Post

ONSITE

1 ERDA Richland Operations Office Programs Division

4 ERDA Richland Operations Office Production and Waste Management Program Division

0. J. Elgert

R. B. Goranson

C. R. Palmer

D. J. Squires

1 ERDA Richland Operations Office Safety and Quality Assurance Div.

J. H. Straub, Director

4 Atlantic Richfield Hanford Co.

R. E. Isaacson

D. C. Nelson

W. W. Shultz

File Copy

2 Westinghouse Hanford Company

R. E. Lerch

G. L. Richardson 
No. of

Copies

53 Battelle-Northwest

T. W. Ambrose

J. W. Bartlett

W. J. Bjorklund

H. T. Blair

W. F. Bonner

D. J. Bradley

J. R. Carrell

N. E. Carter

C. C. Chapman

T. D. Chikalla

R. D. Dierks

J. W. Finnigan

A. A. Garrett

T. A. Golding

M. S. Hanson

W. S. Kelly

Y. B. Katayama

R. S. Kemper

R. P. Marshall

J. L. McElroy

J. E. Mende ?

R. E. Nightingale

D. E. Olesen

A. M. Platt

F. P. Roberts

W. A. Ross

J. L. Rusin

D. H. Siemens

S. C. Slate

R. P. Turcotte

H. H. Van Tuyl

J. H. Westsik, Jr.

L. D. Williams

W. K. Hinegardner

Primary Author (15)

Technical Information (3)

Technical Publications 